\title{
CLOSED 1-FORMS IN TOPOLOGY AND DYNAMICS
}

\author{
MICHAEL FARBER AND DIRK SCHÜTZ
}

\begin{abstract}
This article surveys recent progress of results in topology and dynamics based on techniques of closed one-forms. Our approach allows us to draw conclusions about properties of flows by studying homotopical and cohomological features of manifolds. More specifically we describe a Lusternik - Schnirelmann type theory for closed one-forms, the focusing effect for flows and the theory of Lyapunov one-forms. We also discuss recent results about cohomological treatment of the invariants $\operatorname{cat}(X, \xi)$ and $\operatorname{cat}^{1}(X, \xi)$ and their explicit computation in certain examples.
\end{abstract}

To S.P. Novikov on the occasion of his 70-th birthday

In 1981 S.P. Novikov [36, 39] initiated a generalization of Morse theory which gives topological estimates on the numbers of zeros of closed 1-forms, see also [41, 42. Novikov was motivated by a variety of important problems of mathematical physics, leading, in one way or another, to the problem of finding relations between the topology of the underlying manifold and the number of zeros which closed 1-forms in a specific one-dimensional real cohomology class possess. Recall that a closed 1-form can be viewed as a multi-valued function or functional whose branching behavior is fully characterized by the cohomology class.

In 39 Novikov studies various problems of physics where the motion can be reduced to the principle of an extremal action $S$, which is a multi-valued functional on the space of curves, with the variation $\delta S$ being a well-defined closed 1-form, cf. [38, 37, 40]. Among problems of this kind are the Kirchhoff equations for the motion of a rigid body in ideal fluid, the Leggett equation for the magnetic momentum, and others.

His fundamental idea in [39] was based on a plan to construct a chain complex, now called the Novikov complex, which uses dynamics of the gradient flow in the abelian covering associated with the cohomology class. The dynamics of gradient flows appears traditionally in Morse theory providing a bridge between the critical set of a function and the global ambient topology.

Date: October 24, 2018. 
At present the Novikov theory of closed one-forms is a rapidly developing area of topology which interacts with various mathematical theories. J.Cl. Sikorav [55, 56] was the first who applied Novikov theory in symplectic topology. Hofer and Salamon [28] pioneered exploiting the ideas of Novikov theory in Floer theory. More recent applications of Novikov theory in symplectic topology and Hamiltonian dynamics can be found, for example, in the work of Oh [43], Usher 62] and references mentioned there. Combinatorial group theory is another area where Novikov theory plays an important role; this connection was also discovered by J.-Cl. Sikorav who realized that the Bieri - Neumann - Strebel invariant can be expressed in terms of (a generalized noncommutative) Novikov homology.

Many problems of Novikov theory are still being actively developed in current research. The list of such topics includes (a) constructions of chain complexes (more general than the Novikov complex) which are able to capture the link between topology of the manifold and topology of the set of zeros, (b) various types of inequalities for closed 1-forms (with Morse or Bott type nondegeneracy assumptions), (c) equivariant inequalities and (d) problems about sharpness of these inequalities.

Two recent monographs [18, 47] give expositions of Novikov theory from quite different angles.

Topology of closed 1-forms is a broader research area which together with the Novikov theory studies a new Lusternik - Schnirelmann type theory for closed 1-forms initiated in 2002 in [15]. The latter theory also aims at finding relations between topology of the zero set of a closed one-form and homotopy information, based mainly on the cohomology class of the form. The words "Lusternik - Schnirelmann type" intend to emphasize that no assumptions on the character of zeros are made, unlike the ones which appear in the Novikov theory requiring that all zeros are nondegenerate in the sense of Morse.

Although Lusternik - Schnirelmann theory for closed one-forms shares many common features with the Lusternik - Schnirelmann theory of functions and with the Novikov theory of closed one-forms, it is also very different from both these classical theories. The most striking new phenomenon is based on the fact that in any nonzero cohomology class there always exists a closed 1form having at most one zero (see Theorem 4 below). Hence, the new theory is not merely about the number of zeros but, as we show in this article, about qualitative dynamical properties of smooth flows on manifolds.

Given a smooth flow one wants to "tame" it by a closed 1-form lying in a prescribed cohomology class. More precisely, one wants to find a closed 1form such that the flow is locally decreasing. This idea leads to the notion of a Lyapunov closed one-form generalizing the classical notion of a Lyapunov function. The key question about the existence of Lyapunov closed 1-forms for flows has been resolved in a series of papers of Farber, Kappeler, Latschev and Zehnder [16, 19, 20, 32, 33. Note that the classical theorem of C. 
Conley [4, 5] gives the answer in the special case of the zero cohomology class, i.e. when one deals with Lyapunov functions. When the cohomology class is nontrivial the notion of asymptotic cycle of a flow introduced by Schwartzman [52, 53] plays a crucial role.

A brief account of Lusternik - Schnirelmann theory for closed one-forms can be found in chapter 10 of the book [18] published in 2004. The main idea of this paper is to survey the new results obtained after 2004. To put the material in context we start this survey with a section describing the basic results of the Novikov theory.

In a forthcoming paper [26] we introduce and study the notion of sigma invariants for a finite CW-complex $X$ analogously to the sigma invariants of groups of Bieri et al [1, 2]. These invariants carry information about the finiteness properties of infinite abelian covers of $X$. Both the present survey and [26] have a common main theme: they are based on movability properties of subsets of $X$ with respect to a closed 1-form which are very close in spirit to the idea of Novikov homology.

Table of contents

1. Fundamentals of the Novikov theory

2. The colliding theorem

3. Closed 1-forms on general topological spaces

4. Lyapunov 1-forms for flows

5. Notions of category with respect to a cohomology class

6. Focussing effect

7. Existence of flow-convex neighbourhoods

8. Proof of Theorem 12

9. Topology of the chain recurrent set $R_{\xi}$

10. Proof of Theorem 14

11. Cohomological estimates for $\operatorname{cat}(X, \xi)$

12. Upper bounds for $\operatorname{cat}(X, \xi)$ and relations with the Bieri - Neumann Strebel invariants

13. Homological category weights, estimates for $\operatorname{cat}(X, \xi)$ and calculation of $\operatorname{cat}(X, \xi)$, $\operatorname{cat}^{1}(X, \xi)$ for products of surfaces

\section{Fundamentals of the Novikov theory}

S.P. Novikov [36, 39] suggested a generalization of the classical Morse theory which gives lower bounds on the number of zeros of Morse closed 1-forms. In this section we will give a brief account of the Novikov theory. We touch here only the following selected topics: Novikov inequalities, Novikov numbers, and Novikov Principle leading to the notion of the Novikov complex. 
We refer the reader to the original papers [36, 39] and to the monograph [18] for proofs and more details. Historical information about the development of the subject after [36, 39] as well as bibliographic references can also be found in [18].

1.1. Novikov inequalities. Let $M$ be a smooth manifold. A smooth closed 1-form $\omega$ on $M$ is defined as a smooth section of the cotangent bundle $T^{*} M \rightarrow M$ satisfying $d \omega=0$. By the Poincaré lemma for any simply connected open set $U \subset M$ one has $\omega \mid U=d f_{U}$ where $f_{U}: U \rightarrow \mathbb{R}$ is a smooth function, defined uniquely up to addition of a locally constant function. The zeros of $\omega$ are points $p \in M$ such that $\omega_{p}=0$. If $p$ lies in a simply connected domain $U$ then $\omega_{p}=0$ if and only if $p$ is a critical point of $f_{U}$.

Definition 1. A zero $p \in M$ of a smooth closed 1-form $\omega$ is said to be non-degenerate if it is a non-degenerate critical point of the function $f_{U}$.

Clearly, this property is independent of the choice of the simply connected domain $U$ and the function $f_{U}$.

Definition 2. The Morse index of a non-degenerate zero $p$ of $\omega$ is defined as the Morse index of $p$ viewed as a critical point of $f_{U}$.

We denote the Morse index by ind $(p)$. It takes values $0,1,2, \ldots, n$ where $n=\operatorname{dim} M$.

The main problem of the Novikov theory is as follows. Let $\omega$ be a smooth closed one-form on a closed smooth manifold $M$. Let us additionally assume that $\omega$ is Morse, i.e. all its zeros are nondegenerate in the sense explained above. We denote by $c_{i}(\omega)$ the number of zeros of $\omega$ having Morse index $i$, where $i=0,1, \ldots, n$. One wants to estimate the numbers $c_{i}(\omega)$ in terms of information about the topology of $M$ and of the cohomology class

$$
\xi=[\omega] \in H^{1}(M ; \mathbb{R})
$$

represented by $\omega$.

In the case of classical Morse theory one has $\xi=[\omega]=0$ (i.e. $\omega=d f$ where $f: M \rightarrow \mathbb{R}$ is a smooth function) and the answer is given by the Morse inequalities

$$
c_{i}(\omega) \geq b_{i}(M)+q_{i}(M)+q_{i-1}(M)
$$

where $b_{i}(M)$ denotes the $i$-th Betti number of $M$ and $q_{i}(M)$ denotes the minimal number of generators of the torsion subgroup of $H_{i}(M ; \mathbb{Z})$.

S.P. Novikov [36, 39] introduced generalizations of the numbers $b_{i}(M)$ and $q_{i}(M)$ which depend on the cohomology class $\xi$ of $\omega$ (see (1)) and are denoted $b_{i}(\xi)$ and $q_{i}(\xi)$ correspondingly. We call $b_{i}(\xi)$ the Novikov - Betti number; the number $q_{i}(\xi)$ is the Novikov torsion number. Their definitions will be given below. The Novikov inequality (in its simplest form) states: 
Theorem 1. Let $\omega$ be a smooth closed 1-form on a smooth closed manifold $M$. Assume that all zeros of $\omega$ are nondegenerate. Then

$$
c_{i}(\omega) \geq b_{i}(\xi)+q_{i}(\xi)+q_{i-1}(\xi),
$$

where $\xi=[\omega] \in H^{1}(M ; \mathbb{R})$ denotes the cohomology class represented by $\omega$.

Note that for $\xi=0$ the number $b_{i}(\xi)$ coincides with $b_{i}(M)$ and the number $q_{i}(\xi)$ coincides with $q_{i}(M)$, i.e. the Novikov inequalities (3) contain the Morse inequalities (2) as a special case.

Our goal in this section is to explain the main ideas of the proof of Theorem 1 without intending to present full details. A complete proof can be found in [18], pages $46-48$.

Next we introduce the Novikov ring $\mathbf{N o v}_{\mathbf{k}}$ where $\mathbf{k}$ is a commutative ring. Elements of $\mathbf{N o v}_{\mathbf{k}}$ are formal "power series" of the form

$$
x=\sum_{\gamma \in \mathbb{R}} n_{\gamma} t^{\gamma}
$$

where $t$ is a formal variable, the coefficients are elements of $\mathbf{k}, n_{\gamma} \in \mathbf{k}$, and the exponents $\gamma \in \mathbb{R}$ are arbitrary real numbers satisfying the following condition: for any $c \in \mathbb{R}$ the set

$$
\left\{\gamma \in \mathbb{R} ; n_{\gamma} \neq 0, \gamma>c\right\}
$$

is finite. Equivalently, an element $x \in \mathbf{N o v}_{\mathbf{k}}$ can be represented in the form

$$
x=n_{0} t^{\gamma_{0}}+n_{1} t^{\gamma_{1}}+n_{2} t^{\gamma_{2}}+\ldots
$$

where $n_{i} \in \mathbf{k}, \gamma_{i} \in \mathbb{R}, \gamma_{0}>\gamma_{1}>\gamma_{2}>\ldots$ and $\gamma_{i}$ tends to $-\infty$. In other words, element of the Novikov ring $\mathbf{N o v}_{\mathbf{k}}$ are "Laurent like" power series with integral coefficients and with arbitrary real exponents tending to $-\infty$. Addition in $\mathbf{N o v}_{\mathbf{k}}$ is given by adding the coefficients of powers of $t$ with equal exponents. Multiplication in $\mathbf{N o v}_{\mathbf{k}}$ is given by the formula

$$
\sum n_{\gamma} t^{\gamma} \cdot \sum n_{\gamma}^{\prime} t^{\gamma}=\sum n_{\gamma}^{\prime \prime} t^{\gamma}
$$

where

$$
n_{\gamma}^{\prime \prime}=\sum_{\gamma_{1}+\gamma_{2}=\gamma} n_{\gamma_{1}} n_{\gamma_{2}}^{\prime} .
$$

Note that the last sum contains only finitely many nonzero terms and moreover, the set of all the exponents $\gamma \in \mathbb{R}$ for which $n_{\gamma}^{\prime \prime}$ is nonzero also satisfies the property that the sets (4) are finite.

For simplicity, we will abbreviate the notation $\mathbf{N o v}_{\mathbb{Z}}$ to $\mathbf{N o v}$.

Lemma 1. The ring Nov is a principal ideal domain. Moreover, for any field $\mathbf{k}$ the ring $\mathbf{N o v}_{\mathbf{k}}$ is a field.

The second statement is in fact trivial; the proof of the first statement can be found in 18, page 8 . The credit for Lemma 1 should be mainly given to S.P. Novikov who discovered and stated it without proof. Full proofs appear 
in [28, 44] and [18]. Similar statements concerning related rings of rational functions were discovered in [11, [55].

Now we can explain how one associates to a cohomology class $\xi \in H^{1}(M ; \mathbb{R})$ the Novikov numbers $b_{i}(\xi)$ and $q_{i}(\xi)$. Here we assume that $M$ is a smooth closed manifold although the construction is applicable to finite polyhedra. Consider the group ring $\mathbb{Z}[\pi]$ where $\pi$ denotes the fundamental group $\pi=$ $\pi_{1}\left(X, x_{0}\right)$. The class $\xi$ determines a ring homomorphism

$$
\phi_{\xi}: \mathbb{Z}[\pi] \rightarrow \text { Nov }
$$

defined on a group element $g \in \pi$ by the formula

$$
\phi_{\xi}(g)=t^{\langle\xi, g\rangle} \in \text { Nov. }
$$

Here $\langle\xi, g\rangle \in \mathbb{R}$ is the evaluation of the cohomology class $\xi$ on the homotopy class $g$. Recall that $t$ denote the formal indeterminant of the Novikov ring. Clearly,

$$
\phi_{\xi}\left(g g^{\prime}\right)=t^{\left\langle\xi, g g^{\prime}\right\rangle}=t^{\langle\xi, g\rangle+\left\langle\xi, g^{\prime}\right\rangle}=\phi_{\xi}(g) \phi_{\xi}\left(g^{\prime}\right)
$$

i.e. $\phi_{\xi}$ is multiplicative on group elements. It follows that $\phi_{\xi}$ extends by linearity on the whole group ring as a ring homomorphism. By the wellknown construction homomorphism (5D) defines a local system of left Novmodules over $M$ which we denote by $\mathcal{L}_{\xi}$. The homology $H_{i}\left(M ; \mathcal{L}_{\xi}\right)$ of this local system is a finitely generated module over the Novikov ring Nov. As we know, Nov is a principal ideal domain, and therefore $H_{i}\left(M ; \mathcal{L}_{\xi}\right)$ is a direct sum of a free and a torsion Nov-module.

Definition 3. The Novikov-Betti number $b_{i}(\xi)$ is defined as the rank of the free part of $H_{i}\left(X, \mathcal{L}_{\xi}\right)$. The Novikov torsion number $q_{i}(\xi)$ is defined as the minimal number of generators of the torsion submodule of $H_{i}\left(X ; \mathcal{L}_{\xi}\right)$.

Recall the definition of homology of local system $H_{i}\left(M ; \mathcal{L}_{\xi}\right)$. Consider the universal cover $\tilde{M} \rightarrow M$ and the singular chain complex $C_{*}(\tilde{M})$. The latter is a chain complex of free left modules over the group ring $\mathbb{Z}[\pi]$. One views Nov as a right $\mathbb{Z}[\pi]$-module where

$$
x \cdot g=x \phi_{\xi}(g), \quad \text { where } \quad x \in \text { Nov }, \quad g \in \pi .
$$

Then $H_{i}\left(M ; \mathcal{L}_{\xi}\right)$ is the homology of the chain complex

$$
\operatorname{Nov} \otimes_{\mathbb{Z}[\pi]} C_{*}(\tilde{M}) .
$$

1.2. Novikov and Universal complexes. The main idea of S.P. Novikov in [36, 39] which finally led him to Theorem 1] was the statement that for any Morse closed 1-form $\omega$ on a smooth closed manifold $M$ there exists a chain complex $C^{\omega}$ (which is now known as the Novikov complex) having the following two properties:

(1) $C^{\omega}$ is a free chain complex of Nov-modules and each module $C_{i}^{\omega}$ has a canonical free basis which is in one-to-one correspondence with zeros of $\omega$ having Morse index $i$, where $i=0,1, \ldots, n=\operatorname{dim} M$. 
(2) $C^{\omega}$ is chain homotopy equivalent to the complex $\mathbf{N o v} \otimes_{\mathbb{Z}[\pi]} C_{*}(\tilde{M})$. In particular, the homology $H_{i}\left(C^{\omega}\right)$ is isomorphic to $H_{i}\left(M ; \mathcal{L}_{\xi}\right)$.

This statement, which we call the the Novikov Principle, trivially implies Theorem 1. It should be compared with the classical Morse Principle which claims that for any Morse function on a closed smooth manifold there exists a chain complex $C^{f}$ having the following two properties:

(1) $C^{f}$ is a free chain complex of $\mathbb{Z}[\pi]$-modules and each module $C_{i}^{f}$ has a canonical free basis which is in one-to-one correspondence with critical points of $f$ having Morse index $i$, where $i=0,1, \ldots, n=$ $\operatorname{dim} M$.

(2) $C^{f}$ is chain homotopy equivalent to the chain complex $C_{*}(\tilde{M})$, where $\tilde{M}$ is the universal cover of $M$ and $\pi=\pi_{1}(M)$.

There are several explicit constructions of Morse theory which lead to the complex $C^{f}$. One of them is based on the fact that $M$ admits a celldecomposition with cells in one-to-one correspondence with critical points of $f$. Another well-known construction of $C^{f}$ is based on the Witten deformation of the de Rham complex.

One is naturally led to ask if there exist other ring homomorphisms

$$
\rho: \mathbb{Z}[\pi] \rightarrow \mathcal{R}
$$

distinct from (5), for which the analogue of the Novikov Principle holds. To be more specific, we say that the Novikov Principle is valid for a group $\pi, a$ group homomorphism ${ }^{1} \xi: \pi \rightarrow \mathbb{R}$ and a ring homomorphism $\rho: \mathbb{Z}[\pi] \rightarrow \mathcal{R}$ if for any smooth closed manifold $M$ with $\pi_{1}(M)=\pi$ and for any Morse closed 1-form $\omega$ on $M$ representing the cohomology class $\xi$ there exists a chain complex

$$
C^{\omega}=\left(0 \rightarrow C_{n}^{\omega} \rightarrow C_{n-1}^{\omega} \rightarrow \cdots \rightarrow C_{1}^{\omega} \rightarrow C_{0}^{\omega} \rightarrow 0\right)
$$

of free finitely generated $\mathcal{R}$-modules having the following two properties:

(1) each module $C_{i}^{\omega}$ has a canonical free basis which is in one-to-one correspondence with the zeros of $\omega$ having Morse index $i$;

(2) The complex $C^{\omega}$ is chain homotopy equivalent to $\mathcal{R} \otimes_{\mathbb{Z}[\pi]} C_{*}(\tilde{M})$, where $\mathcal{R}$ is viewed as a left $\mathbb{Z}[\pi]$-module via $\rho$.

A positive answer to this question was given in [12] in the case of rational cohomology classes and in [14] in the general case. Besides (5) the Novikov principle holds for many other ring homomorphisms (7). Moreover, there exists "the largest" such homomorphism which we describe below. The chain complex $C^{\omega}$ in this case is called the Universal complex. This complex lives over a localization of the group ring $\mathbb{Z}[\pi]$.

An element $\alpha \in \mathbb{Z}[\pi]$ will be called $\xi$-negative if $\alpha=\sum n_{j} g_{j}$ (finite sum), where $n_{j} \in \mathbb{Z}$ and $\xi\left(g_{j}\right)<0$ for all $j$. An $m \times m$-matrix $A$ over the group

\footnotetext{
${ }^{1}$ One has $\xi\left(g g^{\prime}\right)=\xi(g)+\xi\left(g^{\prime}\right)$ for $g, g^{\prime} \in \pi$
} 
ring $\mathbb{Z}[\pi]$ will be called $\xi$-negative if all its entries are $\xi$-negative. Consider the class $\Sigma_{\xi}$ of square matrices of the form $\operatorname{Id}+A$, where $A$ is an arbitrary $\xi$-negative square matrix with entries in $\mathbb{Z}[\pi]$.

Note that in the case $\xi=0$ (when one studies Morse functions) the set of $\xi$-negative matrices is empty and so the Cohn localization (8) is just the identity map.

We will use the notion of noncommutative localization developed by P.M. Cohn [3]. The universal Cohn localization of the group ring $\mathbb{Z}[\pi]$ with respect to the class $\Sigma_{\xi}$ is a ring $\Sigma_{\xi}^{-1}(\mathbb{Z}[\pi])$ together with a ring homomorphism

$$
\rho_{\xi}: \mathbb{Z}[\pi] \rightarrow \Sigma_{\xi}^{-1}(\mathbb{Z}[\pi]),
$$

satisfying the following two properties: first, any matrix $\rho_{\xi}(\operatorname{Id}+A)$, where $A$ is $\xi$-negative, is invertible over $\Sigma_{\xi}^{-1}(\mathbb{Z}[\pi])$, and, secondly, it is a universal homomorphism having this property, i.e., for any ring homomorphism $\rho$ : $\mathbb{Z}[\pi] \rightarrow \mathcal{R}$, inverting all matrices of the form $I+A$, where $A$ is $\xi$-negative, there exists a unique ring homomorphism $\phi: \Sigma_{\xi}^{-1}(\mathbb{Z}[\pi]) \rightarrow \mathcal{R}$ such that the following diagram commutes

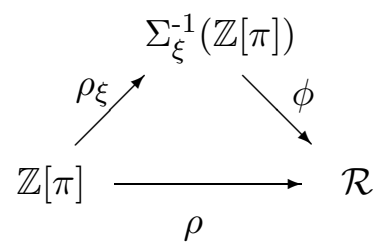

We refer to the book of P.M. Cohn [3], where the existence of localization (8) is proven.

Theorem 2. Let $\pi$ be any group and $\xi \in H^{1}(\pi ; \mathbb{R})$ be a cohomology class. Then the Novikov Principle is valid for the Cohn localization (8).

Farber and Ranicki in [12] proved this theorem in the case of integral cohomology classes; the general case was treated by Farber [13]. The construction of the Universal complex $C^{\omega}$ employs the technique of collapse for chain complexes which is analogous to the well-known operation of combinatorial collapse. This technique was initiated in [12].

One should mention another important closely related ring called the NovikovSikorav completion $\widehat{\mathbb{Z} \pi_{\xi}}$; it was first introduced by J.-Cl. Sikorav [55] who was inspired by the construction of the Novikov ring Nov. One can view $\widehat{\mathbb{Z} \pi_{\xi}}$ as the noncommutative analogue of the Novikov ring. Elements of $\widehat{\mathbb{Z} \pi_{\xi}}$ are represented by formal sums, possibly infinite,

$$
\alpha=\sum n_{i} g_{i}
$$

where $n_{i} \in \mathbb{Z}$ and $g_{i} \in \pi$, satisfying the following condition: for any real number $c \in \mathbb{R}$ the set $\left\{i ; \xi\left(g_{i}\right) \geq c\right\}$ is finite. Compare this with the 
construction of the Novikov ring above. Addition and multiplication are given by the usual formulae; for example, the product of $\alpha=\sum n_{i} g_{i} \in \widehat{\mathbb{Z} \pi_{\xi}}$ and $\beta=\sum m_{j} h_{j} \in \widehat{\mathbb{Z} \pi_{\xi}}$ is given by

$$
\alpha \cdot \beta=\sum_{i, j}\left(n_{i} m_{j}\right)\left(g_{i} h_{j}\right) .
$$

The ring homomorphism $\rho: \mathbb{Z}[\pi] \rightarrow \widehat{\mathbb{Z} \pi_{\xi}}$ is the inclusion. If $A$ is a $\xi$-negative square matrix over the ring $\mathbb{Z}[\pi]$, then the power series

$$
(\operatorname{Id}+A)^{-1}=\operatorname{Id}-A+A^{2}-\ldots
$$

converges in $\widehat{\mathbb{Z} \pi_{\xi}}$ and hence the matrix $I+A$ is invertible in $\widehat{\mathbb{Z} \pi_{\xi}}$. From the universal property of the Cohn localization it follows that there exists a canonical ring homomorphism

$$
\Sigma_{\xi}^{-1}(\mathbb{Z}[\pi]) \rightarrow \widehat{\mathbb{Z} \pi_{\xi}},
$$

extending the inclusion $\mathbb{Z}[\pi] \rightarrow \widehat{\mathbb{Z} \pi_{\xi}}$. This implies that the homomorphism (8) is injective.

It is not known whether (10) is always injective. Intuitively, the image of (10) consists of "rational power series". This is a consequence of the characterization of elements of the Cohn localization as components of solutions of a linear system of equations; cf. [3], Chapter 7 .

1.3. Novikov numbers and the fundamental group. It is obvious that one-dimensional Novikov numbers $b_{1}(\xi)$ and $q_{1}(\xi)$ depend only on the fundamental group $\pi_{1}(X)$ and on the homomorphism of periods $\xi: \pi_{1}(X) \rightarrow \mathbb{R}$. Interestingly there is also a strong inverse dependence, i.e. one may sometimes recover information about the fundamental group while studying $b_{1}(\xi)$ and $q_{1}(\xi)$. The following result shows that nontriviality of the first Novikov Betti number $b_{1}(\xi)$ happens only if the fundamental group $\pi_{1}(X)$ is "large":

Theorem 3 (Farber-Schütz [21]). Let $X$ be a connected finite complex and let $\xi \in H^{1}(X ; \mathbb{R})$ be a nonzero cohomology class. If the first Novikov-Betti number $b_{1}(\xi)$ is positive then $\pi_{1}(X)$ contains a nonabelian free subgroup.

Theorem 3 has the following Corollaries:

Corollary 2. Let $X$ be a connected finite polyhedron having an amenable fundamental group. Then the first Novikov-Betti number vanishes $b_{1}(\xi)=0$ for any $\xi \neq 0 \in H^{1}(X ; \mathbb{R})$.

Corollary 3. Assume that $X$ is a connected finite two-dimensional polyhedron. If the Euler characteristic of $X$ is negative $\chi(X)<0$ then $\pi_{1}(X)$ contains a nonabelian free subgroup. 
Corollary 3 follows from Theorem 3 and from the Euler -Poincarè theorem for the Novikov numbers

$$
\sum_{i=0}^{\infty}(-1)^{i} b_{i}(\xi)=\chi(X) .
$$

The proof of Theorem 3 given in [21] is based on the results of R. Bieri, W. Neumann, R. Strebel [1] and J.-Cl. Sikorav [55].

We refer the reader to articles [45], 46], 48] and [49] for additional information.

\section{The COLLiding THEOREM}

As follows from the discussion of the previous section, the Novikov theory gives bounds from below on the number of distinct zeros which have closed Morse type 1-forms $\omega$ lying in a prescribed cohomology class $\xi \in H^{1}(M ; \mathbb{R})$. The total number of zeros is then at least the sum $\sum_{j} b_{j}(\xi)$ of the Novikov numbers $b_{j}(\xi)$.

If $\omega$ is a closed 1-form representing the zero cohomology class then $\omega=d f$ where $f: M \rightarrow \mathbb{R}$ is a smooth function; in this case $\omega$ must have at least cat $(M)$ geometrically distinct zeros (which are exactly the critical points of the function $f$ ), according to the classical Lusternik-Schnirelmann theory [7] this result requires no assumptions on the nature of the zeros.

Encouraged by the success of the Novikov theory one may ask if it is possible to construct an analogue of the Lusternik - Schnirelmann theory for closed 1-forms. It is quite surprising that in general, with the exception of two situations mentioned above, there are no obstructions for constructing closed 1 -forms possessing a single zero. Hence, for $\xi \neq 0$, the only homotopy invariant $a(M, \xi)$ (depending on the topology of $M$ and on the cohomology class $\xi$ ) such that any closed 1 -form $\omega$ on $M$ with $[\omega]=\xi$ has at least $a(M, \xi)$ zeros is $a(M, \xi)=0$ or $a(M, \xi)=1$.

Theorem 4 (Farber [15], Farber - Schütz [22]). Let $M$ be a closed connected $n$-dimensional smooth manifold, and let $\xi \in H^{1}(M ; \mathbb{R})$ be a nonzero real cohomology class. Then there exists a smooth closed 1-form $\omega$ in the class $\xi$ having at most one zero.

This result suggests that "the Lusternik-Schnirelmann theory for closed 1forms" (if it exists) must have a new character, quite distinct from both the classical Lusternik-Schnirelmann theory of functions and the Novikov theory of closed 1-forms.

Theorem 4 was proven in [15] under an additional assumption that the class $\xi$ is integral, $\xi \in H^{1}(M ; \mathbb{Z})$; see also [18], Theorem 10.1. In full generality it was proven in [22].

Let us mention briefly a similar question. We know that if $\chi(M)=0$ then there exists a nowhere zero 1 -form $\omega$ on $M$. Given $\chi(M)=0$, one may ask 
if it is possible to find a nowhere zero 1 -form $\omega$ on $M$ which is closed, i.e. $d \omega=0$ ? The answer is negative in general. For example, vanishing of the Novikov numbers $b_{j}(\xi)=0$ is a necessary condition for the class $\xi$ to be representable by a closed 1-form without zeros. The full list of necessary and sufficient conditions (in the case $\operatorname{dim} M>5$ ) is given by the theorem of Latour [34].

Theorem 4 was proven in [15] and [18] in the case when the cohomology class $\xi$ is of rank 1 . In this section we follow our recent paper [22] and give the proof of Theorem 4 in the case when $\operatorname{rk}(\xi)>1$.

2.1. Singular foliations of closed one-forms. Let $M$ be a smooth manifold. A smooth closed 1-form $\omega$ with Morse zeros determines a singular foliation $\omega=0$ on $M$. It is a decomposition of $M$ into leaves: two points $p, q \in M$ belong to the same leaf if there exists a path $\gamma:[0,1] \rightarrow M$ with $\gamma(0)=p, \gamma(1)=q$ and $\omega(\dot{\gamma}(t))=0$ for all $t$. Locally, in a simply connected domain $U \subset M$, we have $\left.\omega\right|_{U}=d f$, where $f: U \rightarrow \mathbb{R}$; each connected component of the level set $f^{-1}(c)$ lies in a single leaf. If $U$ is small enough and does not contain the zeros of $\omega$, one may find coordinates $x_{1}, \ldots, x_{n}$ in $U$ such that $f \equiv x_{1}$; hence the leaves in $U$ are the sets $x_{1}=c$. Near such points the singular foliation $\omega=0$ is a usual foliation. On the contrary, if $U$ is a small neighborhood of a zero $p \in M$ of $\omega$ having Morse index $0 \leq k \leq n$, then there are coordinates $x_{1}, \ldots, x_{n}$ in $U$ such that $x_{i}(p)=0$ and the leaves of $\omega=0$ in $U$ are the level sets $-x_{1}^{2}-\cdots-x_{k}^{2}+x_{k+1}^{2}+\cdots+x_{n}^{2}=c$. The leaf $L$ with $c=0$ contains the zero $p$. It has a singularity at $p$ : a neighborhood of $p$ in $L$ is homeomorphic to a cone over the product $S^{k-1} \times S^{n-k-1}$. There are finitely many singular leaves, i.e. the leaves containing the zeros of $\omega$.

We are particularly interested in the singular leaves containing the zeros of $\omega$ having Morse indices 1 and $n-1$. Removing such a zero $p$ locally disconnects the leaf $L$. However globally the complement $L-p$ may or may not be connected.

The singular foliation $\omega=0$ is co-oriented: the normal bundle to any leaf at any nonsingular point has a specified orientation.

We shall use the notion of a weakly complete closed 1-form introduced by G. Levitt [35. A closed 1-form $\omega$ is called weakly complete if it has Morse type zeros and for any smooth path $\sigma:[0,1] \rightarrow M^{*}$ with $\int_{\sigma} \omega=0$ the endpoints $\sigma(0)$ and $\sigma(1)$ lie in the same leaf of the foliation $\omega=0$ on $M^{*}$. Here $M^{*}$ denotes $M-\left\{p_{1}, \ldots, p_{m}\right\}$ where $p_{j}$ are the zeros of $\omega$.

A weakly complete closed 1 -form with $\xi=[\omega] \neq 0$ has no zeros with Morse indices 0 and $n$. According to Levitt [35], any nonzero real cohomology class $\xi \in H^{1}(M ; \mathbb{R})$ can be represented by a weakly complete closed 1-form.

The plan of our proof of Theorem 4 is as follows. We start with a weakly complete closed 1-form $\omega$ lying in the prescribed cohomology class $\xi \in H^{1}(M ; \mathbb{R})$, $\xi \neq 0$. We show that assuming $\operatorname{rk}(\xi)>1$ all leaves of the singular foliation $\omega=0$ are dense (see 92.2 ). We perturb $\omega$ such that the resulting closed 
1-form $\omega^{\prime}$ has a single singular leaf (see 2.3 ). After that we apply the technique of Takens [61] allowing us to collide the zeros in a single (highly degenerate) zero. We first prove Theorem 4 assuming that $n=\operatorname{dim} M>2$; the special case $n=2$ is treated separately later.

2.2. Density of the leaves. In this section we show that if $\omega$ is weakly complete and $\operatorname{rk}(\xi)>1$ then the leaves of $\omega=0$ are dense; moreover, given a point $x \in M$ and a leaf $L \subset M$ of the singular foliation $\omega=0$, there exist two sequences of points $x_{k} \in L$ and $y_{k} \in L$ such that $x_{k} \rightarrow x, y_{k} \rightarrow x$, and

$$
\int_{x}^{x_{k}} \omega>0, \text { while } \int_{x}^{y_{k}} \omega<0 .
$$

The integrals in (11) are calculated along an arbitrary path lying in a small neighborhood of $x$. This can also be expressed by saying that the leaf $L$ approaches $x$ from both the positive and the negative sides. This statement was also observed by G. Levitt [35, p. 645; we give below a different proof. In general the assumptions that $\omega$ has no centers and $\operatorname{rk}(\xi)>1$ do not imply that the leaves of the foliation $\omega=0$ are dense, see the examples in Chapter $11, \S 9.3$ of [18].

Let $\omega$ be a weakly complete closed 1-form in class $\xi$. Consider the covering $\pi: \tilde{M} \rightarrow M$ corresponding to the kernel of the homomorphism of periods $\operatorname{Per}_{\xi}: \pi_{1}(M) \rightarrow \mathbb{R}$, where $\xi=[\omega] \in H^{1}(M ; \mathbb{R})$. Let $H \subset \mathbb{R}$ be the group of periods. The rank of $H$ equals $\operatorname{rk}(\xi)$; since we assume that $\operatorname{rk}(\xi)>1$, the group $H$ is dense in $\mathbb{R}$. The group of periods $H$ acts on the covering space $\tilde{M}$ as the group of covering transformations. We have $\pi^{*} \omega=d F$ where $F: \tilde{M} \rightarrow \mathbb{R}$ is a smooth function. The leaves of the singular foliation $\omega=0$ are the images under the projection $\pi$ of the level sets $F^{-1}(c)$; this property follows from the weak completeness of $\omega$, see [35], Proposition II.1. For any $g \in H$ and $x \in \tilde{M}$ one has

$$
F(g x)-F(x)=g \in \mathbb{R} .
$$

Let $L=\pi\left(F^{-1}(c)\right)$ be a leaf and let $x \in M$ be an arbitrary point. Our goal is to show that $x$ lies in the closure $\bar{L}$ of $L$. Let $U \subset M$ be a path-connected neighborhood of $x$ which is assumed to be "small" in the following sense: $\left.\xi\right|_{U}=0$. We want to show that $U \cap L$ contains a point $y$ satisfying $\int_{x}^{y} \omega>0$ where the integral is calculated along a curve in $U$.

Consider a lift $\tilde{x} \in \tilde{M}, \pi(\tilde{x})=x$. Let $\tilde{U}$ be a neighborhood of $\tilde{x}$ which is mapped by $\pi$ homeomorphically onto $U$. We claim that the set of values $F(\tilde{U}) \subset \mathbb{R}$ contains an interval $(a-\epsilon, a+\epsilon)$ where $a=F(\tilde{x})$ and $\epsilon>0$. This claim is obvious if $\tilde{x}$ is not a critical point of $F$ since in this case one may choose the coordinates $x_{1}, \ldots, x_{n}$ around $\tilde{x}$ such that $F(x)=a+x_{1}$. In the case when $\tilde{x}$ is a critical point of $F$, one may choose the coordinates $x_{1}, \ldots, x_{n}$ near the point $\tilde{x} \in \tilde{M}$ such that $F(x)$ is given by $a \pm x_{1}^{2} \pm x_{2}^{2}+\cdots+$ $\pm x_{n}^{2}$ and our claim follows since we know that the Morse index is distinct from 0 and $n$. 


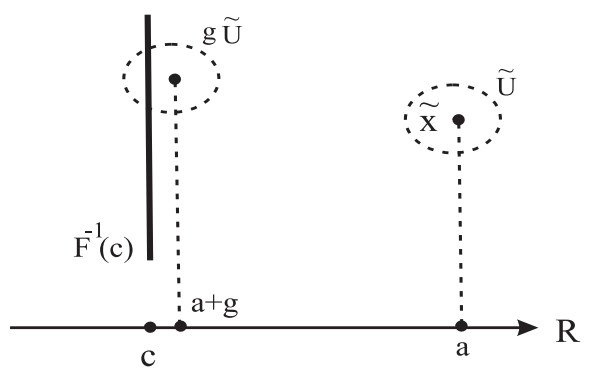

Because of the density of the group of translations $H \subset \mathbb{R}$ one may find $g \in H$ such that the real number $F(g \tilde{x})=F(\tilde{x})+g=a+g$ lies in the interval $(c, c+\epsilon)$. Then we obtain

$$
c \in(a+g-\epsilon, a+g) \subset g+F(\tilde{U})=F(g \tilde{U}) .
$$

We see that the sets $F^{-1}(c)$ and $g \tilde{U}$ have a common point $\tilde{y}$. The point $y=\pi(\tilde{y}) \in U \cap L$ has the required property $\int_{x}^{y} \omega>0$.

2.3. Modification. Our next goal is to replace $\omega$ by a Morse closed 1-form $\omega^{\prime}$ which has the property that all its zeros lie on the same singular leaf of the singular foliation $\omega^{\prime}=0$. In this section we assume that $n=\operatorname{dim} M>2$. Let $\omega$ be a weakly complete Morse closed 1-form in class $\xi$ where $\operatorname{rk}(\xi)>1$. Let $p_{1}, \ldots, p_{m} \in M$ be the zeros of $\omega$. For each $p_{j}$ choose a small neighborhood $U_{j} \ni p_{j}$ and local coordinates $x_{1}, \ldots, x_{n}$ in $U_{j}$ such that $x_{i}\left(p_{j}\right)=0$ for $i=1, \ldots, n$ and

(14) $\left.\omega\right|_{U_{j}}=d f_{j}$, where $f_{j}=-x_{1}^{2}-\cdots-x_{m_{j}}^{2}+x_{m_{j}+1}^{2}+\cdots+x_{n}^{2}$.

Here $m_{j}$ denotes the Morse index of $p_{j}$. We assume that the ball $\sum_{i=1}^{n} x_{i}^{2} \leq 1$ is contained in $U_{j}$ and that $U_{j} \cap U_{j^{\prime}}=\emptyset$ for $j \neq j^{\prime}$. Denote by $W_{j}$ the open ball $\sum_{i=1}^{n} x_{i}^{2}<1$.

Let $\phi:[0,1] \rightarrow[0,1]$ be a smooth function with the following properties: (a) $\phi \equiv 0$ on $[3 / 4,1]$; (b) $\phi \equiv \epsilon>0$ on $[0,1 / 2]$; (c) $-1<\phi^{\prime} \leq 0$. Such a function

exists assuming that $\epsilon>0$ is small enough. (a), (b), (c) imply that

$$
\phi^{\prime}(r)>-2 r, \text { for } r>0 .
$$


We replace the closed 1 -form $\omega$ by

$$
\omega^{\prime}=\omega-\sum_{j=1}^{m} \mu_{j} \cdot d g_{j}
$$

where $g_{j}: M \rightarrow \mathbb{R}$ is a smooth function with support in $U_{j}$. In the coordinates $x_{1}, \ldots, x_{n}$ of $U_{j}$ (see above) the function $g_{j}$ is given by $g_{j}(x)=\phi(\|x\|)$. The parameters $\mu_{j} \in[-1,1]$ appearing in (16) are specified later.

One has $\omega \equiv \omega^{\prime}$ on $M-\cup_{j} U_{j}$ and near the zeros of $\omega$. Let us show that $\omega^{\prime}$ has no additional zeros. We have $\left.\omega^{\prime}\right|_{U_{j}}=d\left(f_{j}-\mu_{j} g_{j}\right)$ (where $f_{j}$ is defined in (14)) and

$$
\frac{\partial}{\partial x_{i}}\left(f_{j}-\mu_{j} g_{j}\right)= \pm 2 x_{i}-\mu_{j} \phi^{\prime}(\|x\|) \frac{x_{i}}{\|x\|}
$$

If this partial derivative vanishes and $x_{i} \neq 0$ then $\phi^{\prime}(r)= \pm 2 r \mu_{j}^{-1}$ which may happen only for $r=\|x\|=0$ according to (15).

We now show how to choose the parameters $\mu_{j}$ so that the closed 1-form $\omega^{\prime}$ given by (16) has a unique singular leaf. Let $L$ be a fixed nonsingular leaf of $\omega=0$. Since $L$ is dense in $M$ (see \$2.2) for any $j=1, \ldots, m$ the intersection $L \cap U_{j}$ contains infinitely many connected components approaching $p_{j}$ from below and from above and the function $f_{j}$ is constant on each of them. We

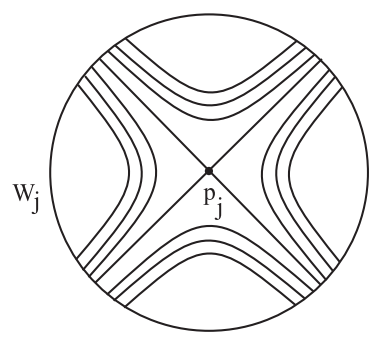

say that a subset $T_{c} \subset L \cap W_{j}$ is a level set if $T_{c}=f_{j}^{-1}(c) \cap W_{j}$ for some $c \in \mathbb{R}$. Note that $f_{j}\left(p_{j}\right)=0$. The level set $c=0$ contains the zero $p_{j}$; it is homeomorphic to the cone over the product $S^{m_{j}-1} \times S^{n-m_{j}-1}$. Each level set $T_{c}$ with $c<0$ is diffeomorphic to $S^{m_{j}-1} \times D^{n-m_{j}}$ and each level set $T_{c}$ with $c>0$ is diffeomorphic to $D^{m_{j}} \times S^{n-m_{j}-1}$. Recall that $m_{j}$ denotes the Morse index of $p_{j}$.

Let $\mathcal{V}_{j}=f_{j}\left(L \cap W_{j}\right) \subset \mathbb{R}$ denote the set of values of $f_{j}$ on different level sets belonging to the leaf $L$. The zero 0 does not lie in $\mathcal{V}_{j}$ since we assume that the leaf $L$ is nonsingular. However, according to the result proven in $\$ 2.2$, the zero $0 \in \mathbb{R}$ is a limit point of $\mathcal{V}_{j}$ and, moreover, the closure of either of the sets $\mathcal{V}_{j} \cap(0, \infty)$ and $\mathcal{V}_{j} \cap(-\infty, 0)$ contains $0 \in \mathbb{R}$.

For the modification $\omega^{\prime}$ (given by (16)) one has $\left.\omega^{\prime}\right|_{U_{j}}=d h_{j}$ where $h_{j}=$ $f_{j}-\mu_{j} g_{j}$. The level sets $T_{c}^{\prime}$ for $h_{j}$ are defined as $h_{j}^{-1}(c) \cap W_{j}$. Clearly $T_{c}^{\prime}$ is 
given by the equation

$$
f_{j}(x)=\mu_{j} \phi(\|x\|)+c, \quad x \in W_{j} .
$$

Hence for $\|x\| \geq 3 / 4$ this is the same as $T_{c}$; for $\|x\| \leq 1 / 2$ the level set $T_{c}^{\prime}$ coincides with $T_{c+\mu_{j} \epsilon}$. In the ring $1 / 2 \leq\|x\| \leq 3 / 4$ the level set $T_{c}^{\prime}$ is homeomorphic to a cylinder.

The following figure illustrates the distinction between the level sets $T_{c}$ and $T_{c}^{\prime}$ in the case $\mu_{j}>0$.

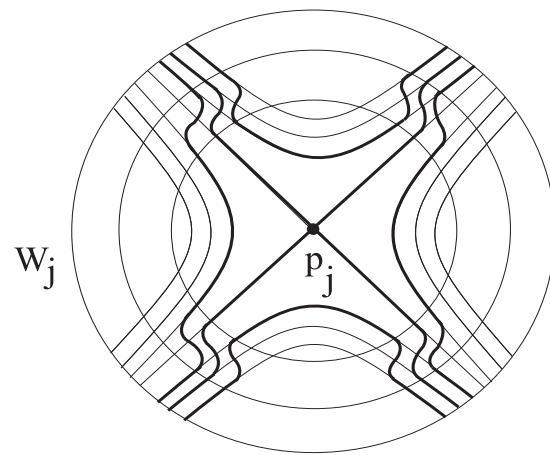

Case $\mu_{\mathrm{j}}>0$.

Examine the changes which the leaf $L$ undergoes when we replace $\omega$ by $\omega^{\prime}$. Here we view $L$ with the leaf topology; it is the topology induced on $L$ from the covering $\tilde{M}$ using an arbitrary lift $L \rightarrow \tilde{M}$. First, let us assume that: (1) the Morse index $m_{j}$ satisfies $m_{j}<n-1$; (2) the coefficient $\mu_{j}>0$ is positive; $(3)$ the number $-\epsilon \mu_{j}$ lies in the set $\mathcal{V}_{j}$. Then the complement

$$
L-\bigcup_{\substack{c \in \mathcal{V}_{j} \\-\epsilon \mu_{j}<c<0}} T_{c}
$$

is connected and it lies in a single leaf $L^{\prime}$ of the singular foliation $\omega^{\prime}=0$. We see that the new leaf $L^{\prime}$ is obtained from $L$ by infinitely many surgeries. Namely, each level set $T_{c} \subset L$, where $c \in \mathcal{V}_{j}$ satisfies $-\epsilon \mu_{j}<c<0$, is removed and replaced by a copy of $D^{m_{j}} \times S^{n-m_{j}-1}$; besides, the set $T_{c} \subset L$ where $c=-\epsilon \mu_{j}$, is removed and gets replaced by a cone over the product $S^{m_{j}-1} \times S^{n-m_{j}-1}$. Hence the new leaf $L^{\prime}$ contains the zero $p_{j}$.

Let us now show how one may modify the above construction in the case $m_{j}=n-1$. Since $n>2$ we have in this case $n-m_{j}-1<n-2$; hence removing the sphere $S^{n-m_{j}-1}$ from the leaf $L$ does not disconnect $L$. We shall assume that the coefficient $\mu_{j}$ is negative and that the number $-\epsilon \mu_{j}$ lies in $\mathcal{V}_{j} \subset \mathbb{R}$. The complement

$$
L-\bigcup_{\substack{c \in \mathcal{V}_{j} \\ 0<c<-\epsilon \mu_{j}}} T_{c}
$$


is connected and it lies in a single leaf $L^{\prime}$ of the singular foliation $\omega^{\prime}=0$. Clearly, $L^{\prime}$ is obtained from $L$ by removing the level sets $T_{c}$ where $c \in \mathcal{V}_{j}$ satisfies $0<c<-\epsilon \mu_{j}$ (each such $T_{c}$ is diffeomorphic to $D^{m_{j}} \times S^{n-m_{j}-1}$ ) and by replacing them by copies of $S^{m_{j}-1} \times D^{n-m_{j}}$. In addition, the set $T_{c} \subset L$ where $c=-\epsilon \mu_{j}$, is removed and is replaces by a cone over the product $S^{m_{j}-1} \times S^{n-m_{j}-1}$.

We see that $L^{\prime}$ is a leaf of the singular foliation $\omega^{\prime}=0$ containing all the zeros $p_{1}, \ldots, p_{m}$.

2.4. Proof of Theorem 4. Below we assume that $\operatorname{rk}(\xi)>1$. The case $\operatorname{rk}(\xi)=1$ is covered by Theorem 2.1 from [15].

The results of the preceding sections allow us to complete the proof of Theorem 4 in the case $n=\operatorname{dim} M>2$. Indeed, we showed in 92.3 how to construct a Morse closed 1-form $\omega^{\prime}$ lying in the prescribed cohomology class $\xi$ such that all zeros of $\omega^{\prime}$ are Morse and belong to the same singular leaf $L^{\prime}$ of the singular foliation $\omega^{\prime}=0$. Now we may apply the colliding technique of F. Takens [61], pages 203-206. Namely, we may find a piecewise smooth tree $\Gamma \subset L^{\prime}$ containing all the zeros of $\omega^{\prime}$. Let $U \subset M$ be a small neighborhood of $\Gamma$ which is diffeomorphic to $\mathbb{R}^{n}$. We may find a continuous map $\Psi: M \rightarrow M$ with the following properties:

$\Psi(\Gamma)$ is a single point $p \in \Gamma$;

$\left.\Psi\right|_{M-\Gamma}$ is a diffeomorphism onto $M-p$;

$\Psi(U)=U$

$\Psi$ is the identity map on the complement of a small neighborhood $V \subset M$ of $\Gamma$ where the closure $\bar{V}$ is contained in $U$.

Consider a smooth function $f: U \rightarrow \mathbb{R}$ such that $d f=\left.\omega^{\prime}\right|_{U}$; it exists and is unique up to a constant. The function $g=f \circ \Psi^{-1}: U \rightarrow \mathbb{R}$ is well-defined (since $\left.f\right|_{\Gamma}$ is constant). $g$ is continuous by the universal property of the quotient topology. Moreover, $g$ is smooth on $M-p$. Applying Theorem 2.7 from [61], we see that we can replace $g$ by a smooth function $h: U \rightarrow \mathbb{R}$ having a single critical point at $p$ and such that $h=f$ on $U-\bar{V}$.

Let $\omega^{\prime \prime}$ be a closed 1-form on $M$ given by

$$
\left.\omega^{\prime \prime}\right|_{M-\bar{V}}=\left.\omega^{\prime}\right|_{M-\bar{V}} \quad \text { and }\left.\quad \omega^{\prime \prime}\right|_{U}=d h .
$$

Clearly $\omega^{\prime \prime}$ is a smooth closed 1-form on $M$ having no zeros in $M-\{p\}$. Moreover, $\omega^{\prime \prime}$ lies in the cohomology class $\xi=\left[\omega^{\prime}\right]$ (since any loop in $M$ is homologous to a loop in $M-\bar{V}$ ).

Now we prove Theorem 4 the case $n=2$. We shall replace the construction of $\$ 2.3$ (which requires $n>2$ ) by a direct construction. The final argument using the Takens' technique [61] remains the same.

Let $M$ be a closed surface and let $\xi \in H^{1}(M ; \mathbb{R})$ be a nonzero cohomology class. We can split $M$ into a connected sum

$$
M=M_{1} \sharp M_{2} \sharp \ldots \sharp M_{k}
$$


where each $M_{j}$ is a torus or a Klein bottle and such that the cohomology class $\xi_{j}=\left.\xi\right|_{M_{j}} \in H^{1}\left(M_{j} ; \mathbb{R}\right)$ is nonzero. Let $\omega_{j}$ be a closed 1-form on $M_{j}$ lying in the class $\xi_{j}$ and having no zeros; obviously such a form exists. $\S 9.3 .2$ of [18] describes the construction of connected sum of closed 1-forms on surfaces. Each connecting tube contributes two zeros. In fact there are three different ways of forming the connected sum, they are denoted by A, $\mathrm{B}, \mathrm{C}$ on Figure 9.8 in [18. In the type $\mathrm{C}$ connected sum the zeros lie on the same singular leaf. Hence by using the type $\mathrm{C}$ connected sum operation we get a closed 1-form $\omega$ on $M$ having $2 k-2$ zeros which all lie on the same singular leaf of the singular foliation $\omega=0$. The colliding argument based on the technique of Takens 61] applies as in the case $n>2$ and produces a closed 1 -form with at most one zero lying in class $\xi$.

\section{Closed OnE-Forms on General topological SPACES}

Since closed one-forms are central for our constructions, it will be convenient to operate with the notion of a closed one-form defined on general topological spaces. This notion will allow us to deal with spaces more general than smooth manifolds. The calculus of closed one-forms on topological spaces is very similar to that of smooth closed one-forms on manifolds: one may integrate any closed one-form along a path; the integral depends only on the homotopy class of the path; any closed one-form represents a onedimensional cohomology class and any continuous function $f$ determines an exact closed one-form $d f$, the differential of $f$.

In this section we recall the basic definitions referring to the book [18] for proofs and more details.

\subsection{Basic definitions.}

Definition 4. A continuous closed 1-form $\omega$ on a topological space $X$ is defined as a collection $\left\{f_{U}\right\}_{U \in \mathcal{U}}$ of continuous real-valued functions $f_{U}$ : $U \rightarrow \mathbb{R}$, where $\mathcal{U}=\{U\}$ is an open cover of $X$, such that for any pair $U, V \in \mathcal{U}$ the difference

$$
\left.f_{U}\right|_{U \cap V}-\left.f_{V}\right|_{U \cap V}: U \cap V \rightarrow \mathbb{R}
$$

is a locally constant function. Another such collection $\left\{g_{V}\right\}_{V \in \mathcal{V}}$ (where $\mathcal{V}$ is another open cover of $X$ ) defines an equivalent closed 1-form if the union collection

$$
\left\{f_{U}, g_{V}\right\}_{U \in \mathcal{U}, V \in \mathcal{V}}
$$

is a closed 1-form (in the sense of the above definition), i.e., if for any $U \in \mathcal{U}$ and $V \in \mathcal{V}$, the function $f_{U}-g_{V}$ is locally constant on $U \cap V$.

Let $\phi: Y \rightarrow X$ be a continuous map and let $\omega$ be a continuous closed 1-form on $X$. Then the induced closed 1 -form $\phi^{*} \omega$ is defined as follows. Let $\omega=$ $\left\{f_{U}\right\}_{U \in \mathcal{U}}$, where $\mathcal{U}$ is an open cover of $X$. The family $\phi^{-1} \mathcal{U}=\left\{\phi^{-1}(U)\right\}_{U \in \mathcal{U}}$ 
is an open cover of $Y$ and the functions $f_{U} \circ \phi$ define a continuous closed 1-form with respect to the cover $\phi^{-1} \mathcal{U}$.

As an example consider an open cover $\mathcal{U}=\{X\}$ consisting of the whole space $X$. Then any continuous function $f: X \rightarrow \mathbb{R}$ defines a closed 1-form on $X$, which is denoted by $d f$. For two continuous functions $f, g: X \rightarrow \mathbb{R}$, $d f=d g$ holds if and only if the difference $f-g: X \rightarrow \mathbb{R}$ is locally constant.

3.2. Integration. One may integrate continuous closed 1-forms along continuous paths. Let $\omega$ be a continuous closed 1-form on $X$ given by a collection of continuous real-valued functions $\left\{f_{U}\right\}_{U \in \mathcal{U}}$ with respect to an open cover $\mathcal{U}$ of $X$. Let $\gamma:[0,1] \rightarrow X$ be a continuous path. The line integral $\int_{\gamma} \omega$ is defined as follows. Find a subdivision $t_{0}=0<t_{1}<\cdots<t_{N}=1$ of the interval $[0,1]$ such that for any $i$ the image $\gamma\left[t_{i}, t_{i+1}\right]$ is contained in a single open set $U_{i} \in \mathcal{U}$. Then we define

$$
\int_{\gamma} \omega=\sum_{i=0}^{N-1}\left[f_{U_{i}}\left(\gamma\left(t_{i+1}\right)\right)-f_{U_{i}}\left(\gamma\left(t_{i}\right)\right)\right] .
$$

The standard argument shows that the integral (19) does not depend on the choice of the subdivision and the open cover $\mathcal{U}$.

For any pair of continuous paths $\gamma, \gamma^{\prime}:[0,1] \rightarrow X$ with common beginning $\gamma(0)=\gamma^{\prime}(0)$ and common end points $\gamma(1)=\gamma^{\prime}(1)$, it holds that

$$
\int_{\gamma} \omega=\int_{\gamma^{\prime}} \omega
$$

provided that $\gamma$ and $\gamma^{\prime}$ are homotopic relative to the boundary.

3.3. Cohomology class of a closed one-form. Any continuous closed 1 -form $\omega$ defines the homomorphism of periods

$$
\pi_{1}\left(X, x_{0}\right) \rightarrow \mathbb{R}, \quad[\gamma] \mapsto \int_{\gamma} \omega \in \mathbb{R}
$$

given by integration along closed loops $\gamma:[0,1] \rightarrow X$ with $\gamma(0)=x_{0}=\gamma(1)$. The image of this homomorphism is a subgroup of $\mathbb{R}$ whose rank is called the rank of $\omega$ and is denoted $\operatorname{rk}(\omega)$.

Recall that a topological space $X$ is homologically locally $n$-connected if for every point $x \in X$ and any neighborhood $U$ of $x$ there exists a neighborhood $V$ of $x$ in $U$ such that the induced homomorphism of the reduced integral singular homology $\tilde{H}_{q}(V) \rightarrow \tilde{H}_{q}(U)$ is trivial for all $q \leq n$. $X$ is locally path connected iff it is homologically locally 0 -connected.

Lemma 4. Let $X$ be a locally path-connected topological space. A continuous closed 1-form $\omega$ on $X$ equals df for a continuous function $f: X \rightarrow \mathbb{R}$ if and only if for any choice of the base point $x_{0} \in X$ the homomorphism of periods (20) determined by $\omega$ is trivial. 
Proof. If $\omega=d f$, then $\int_{\gamma} \omega=f(q)-f(p)$ holds for any path $\gamma$ in $X$, where $q=\gamma(1), p=\gamma(0)$. Hence $\int_{\gamma} \omega=0$ if $\gamma$ is a closed loop.

Conversely, assume that the homomorphism of periods (20) is trivial. Our assumption about $X$ implies that connected components of $X$ are open and path connected. In each connected component of $X$ choose a base point $x_{i} \in X$. One defines a continuous function $f: X \rightarrow \mathbb{R}$ by

$$
f(x)=\int_{x_{i}}^{x} \omega
$$

here $x$ and $x_{i}$ belong the same connected component of $X$ and the integration is taken along an arbitrary path connecting $x_{i}$ to $x$. The result of integration is independent of the choice of the path because of our assumption that the homomorphism of periods (20) is trivial. Assume that $\omega$ is given by a collection of continuous functions $f_{U}: U \rightarrow \mathbb{R}$ with respect to an open cover $\{U\}$ of $X$. Then for any two points $x, y$ lying in the same connected component of $U$,

$$
f(y)-f(x)=\int_{x}^{y} \omega=f_{U}(y)-f_{U}(x)
$$

here the line integral is understood along any continuous path connecting $x$ and $y$. This shows that the function $f-f_{U}$ is locally constant on $U$. Hence $d f=\omega$.

Any continuous closed 1-form $\omega$ on a topological space $X$ defines a singular cohomology class $[\omega] \in H^{1}(X ; \mathbb{R})$. It is defined by the homomorphism of periods (20) viewed as an element of $\operatorname{Hom}\left(H_{1}(X) ; \mathbb{R}\right)=H^{1}(X ; \mathbb{R})$. As follows from the above lemma, two continuous closed 1-forms $\omega$ and $\omega^{\prime}$ on $X$ lie in the same cohomology class $[\omega]=\left[\omega^{\prime}\right]$ if and only if their difference $\omega-\omega^{\prime}$ equals $d f$, where $f: X \rightarrow \mathbb{R}$ is a continuous function. Here we assume that $X$ is locally path-connected.

Lemma 5. Let $X$ be a paracompact Hausdorff homologically locally 1-connected topological space. Then any singular cohomology class $\xi \in H^{1}(X ; \mathbb{R})$ may be realized by a continuous closed 1-form on $X$.

\section{LYAPUNOV 1-FORMS FOR FLOWS}

C. Conley [4], [5] showed that any continuous flow $\Phi: X \times \mathbb{R} \rightarrow X$ on a compact metric space $X$ "decomposes" into a chain recurrent flow and a gradient-like flow. More precisely, he proved the existence of a continuous function $L: X \rightarrow \mathbb{R}$ which (i) decreases along any orbit of the flow in the complement $X-R$ of the chain recurrent set $R \subset X$ of $\Phi$ and (ii) is constant on the connected components of $R$. Such a function $L$ is called a Lyapunov function for $\Phi$. Conley's existence result plays a fundamental role in his program of understanding general flows as collections of isolated invariant sets linked by heteroclinic orbits. 
A more general notion of a Lyapunov 1-form was introduced in the paper [16]. Lyapunov 1-forms, compared to Lyapunov functions, allow us to go one step further and to analyze the flow within the chain-recurrent set $R$ as well. Lyapunov 1-forms provide an important tool in applying methods of homotopy theory to dynamical systems.

The problem of the existence of continuous Lyapunov 1-forms was first addressed in paper [19], in the generality of compact metric spaces, continuous flows and continuous closed 1-forms. In this section we present the result of [20] dealing with the smooth version of the problem: we are interested in constructing smooth Lyapunov 1-forms for smooth flows on smooth manifolds. These conditions are formulated in terms of homological properties of the flow; in particular, we use Schwartzman's asymptotic cycles $\mathcal{A}_{\mu}(\Phi) \in H_{1}(M ; \mathbb{R})$ of the flow.

We would like to refer the reader to related work by J. Latschev [32, 33].

Let $V$ be a smooth vector field on a smooth manifold $M$. Assume that $V$ generates a continuous flow $\Phi: M \times \mathbb{R} \rightarrow M$ and $Y \subset M$ is a closed, flow-invariant subset.

Definition 5. A smooth closed 1-form $\omega$ on $M$ is called a Lyapunov 1-form for the pair $(\Phi, Y)$ if it has the following properties:

$(\Lambda 1)$ The function $\iota_{V}(\omega)=\omega(V)$ is negative on $M-Y$.

$\left.(\Lambda 2) \omega\right|_{Y}=0$ and there exists a smooth function $f: U \rightarrow \mathbb{R}$ defined on an open neighborhood $U$ of $Y$ such that $\left.\omega\right|_{U}=d f$.

The above definition is a modification of the notion of a Lyapunov 1-form introduced in $\S 6$ of [16]. Definition 5 can also be compared with the definition of a Lyapunov 1-form in the continuous setting which was introduced in [19]. Condition $(\Lambda 1)$ above is slightly stronger than condition (L1) of Definition 1 in [19]. Condition $(\Lambda 2)$ is similar to condition (L2) of Definition 1 from [19] although they are not equivalent.

There exist several natural alternatives for condition $(\Lambda 2)$. One of them is:

$\left(\Lambda 2^{\prime}\right)$ The 1 -form $\omega$, viewed as a map $\omega: M \rightarrow T^{*}(M)$, vanishes on $Y$.

It is clear that $(\Lambda 2)$ implies $\left(\Lambda 2^{\prime}\right)$. We can show that the converse is true under some additional assumptions:

Lemma 6. If the de Rham cohomology class $\xi$ of $\omega$ is integral, $\xi=[\omega] \in$ $H^{1}(M ; \mathbb{Z})$, then conditions $\left(\Lambda 2^{\prime}\right)$ and $(\Lambda 2)$ are equivalent.

Proof. Clearly we only need to show that $\left(\Lambda 2^{\prime}\right)$ implies $(\Lambda 2)$. Since $\xi$ is integral, there exists a smooth map $\phi: M \rightarrow S^{1}$ such that $\omega=\phi^{*}(d \theta)$, where $d \theta$ is the standard angular 1-form on the circle $S^{1}$. Let $\alpha \in S^{1}$ be a regular value of $\phi$. Assuming that $\left(\Lambda 2^{\prime}\right)$ holds, it then follows that $U=M-\phi^{-1}(\alpha)$ is an open neighborhood of $Y$. Clearly $\left.\omega\right|_{U}=d f$ where $f: U \rightarrow \mathbb{R}$ is a smooth function which is related to $\phi$ by $\phi(x)=\exp (i f(x))$ for any $x \in U$. Hence $(\Lambda 2)$ holds. 
Lemma 7. Conditions $\left(\Lambda 2^{\prime}\right)$ and $(\Lambda 2)$ are equivalent if $Y$ is a Euclidean Neighborhood Retract (ENR).

Proof. Again, we only have to establish $\left(\Lambda 2^{\prime}\right) \Rightarrow(\Lambda 2)$. Since $Y$ is an ENR it admits an open neighborhood $U \subset M$ such that the inclusion $i_{U}: U \rightarrow M$ is homotopic to $i_{Y} \circ r$, where $i_{Y}: Y \rightarrow M$ is the inclusion and $r: U \rightarrow Y$ is a retraction (see [6], Chapter $4, \S 8$, Corollary 8.7). Pick a base point $x_{j}$ in every path-connected component $U_{j}$ of $U$ and define a smooth function $f_{j}: U_{j} \rightarrow \mathbb{R}$ by

$$
f_{j}(x)=\int_{x_{j}}^{x} \omega, \quad x \in U_{j} .
$$

The latter integral is independent of the choice of the integration path in $U_{j}$ connecting $x_{j}$ with $x$. This claim is equivalent to the vanishing of the integral $\int_{\gamma} \omega$ for any closed loop $\gamma$ lying in $U$. To show this, we apply the retraction to see that $\gamma$ is homotopic in $M$ to the loop $\gamma_{1}=r \circ \gamma$, which lies in $Y$; thus we obtain $\int_{\gamma} \omega=\int_{\gamma_{1}} \omega=0$ because of $\left(\Lambda 2^{\prime}\right)$. It is clear that the functions $f_{j}$ together determine a smooth function $f: U \rightarrow \mathbb{R}$ with $d f=\left.\omega\right|_{U}$

A class of interesting examples of Lyapunov 1-forms can be obtained as follows. Let $\omega$ be a smooth closed 1-form on a closed Riemannian manifold $M$. Consider the negative gradient vector field $V$ of $\omega$, i.e., $\langle V, X\rangle=-\omega(X)$ for any vector field $X$ on $M$ where $\langle\cdot, \cdot\rangle$ denotes the Riemannian metric. Denote by $\Phi$ the flow induced by the vector field $V$ and by $Y$ the set of zeros of $\omega$. Then clearly conditions $(\Lambda 1)$ and $\left(\Lambda 2^{\prime}\right)$ are satisfied. If either the cohomology class of $\omega$ is integral or $Y$ is an ENR, then (by the two lemmas above) $\omega$ is a Lyapunov 1 -form for the pair $(\Phi, Y)$.

The main goal of this section is to specify topological conditions which guarantee that for a given vector field $V$ on $M$ and for a prescribed cohomology class $\xi \in H^{1}(M ; \mathbb{R})$ there exists a Lyapunov 1-form $\omega$ of the flow of $V$ with $[\omega]=\xi$.

Asymptotic cycles of Schwartzman. Let $M$ be a closed smooth manifold and let $V$ be a smooth vector field. Let $\Phi: M \times \mathbb{R} \rightarrow M$ be the flow generated by $V$. Consider a Borel measure $\mu$ on $M$ which is invariant under $\Phi$. According to S. Schwartzman [52], these data determine a real homology class

$$
\mathcal{A}_{\mu}=\mathcal{A}_{\mu}(\Phi) \in H_{1}(M ; \mathbb{R})
$$

called the asymptotic cycle of the flow $\Phi$ corresponding to the measure $\mu$. The class $\mathcal{A}_{\mu}$ is defined as follows. For a de Rham cohomology class $\xi \in$ $H^{1}(M ; \mathbb{R})$ the evaluation $\left\langle\xi, \mathcal{A}_{\mu}\right\rangle \in \mathbb{R}$ is given by the integral

$$
\left\langle\xi, \mathcal{A}_{\mu}\right\rangle=\int_{M} \iota_{V}(\omega) d \mu
$$


where $\omega$ is a closed 1-form in the class $\xi$. Note that $\left\langle\xi, \mathcal{A}_{\mu}\right\rangle$ is well-defined, i.e., it depends only on the cohomology class $\xi$ of $\omega$; see [52], page 277 . Indeed, when we replace $\omega$ by $\omega^{\prime}=\omega+d f$, where $f: M \rightarrow \mathbb{R}$ is a smooth function, the integral in (21) gets changed by the quantity

$$
\int_{M} V(f) d \mu=\lim _{s \rightarrow 0} \frac{1}{s} \int_{M}\{f(x \cdot s)-f(x)\} d \mu(x) .
$$

Here $V(f)$ denotes the derivative of $f$ in the direction of the vector field $V$ and $x \cdot s$ stands for the flow $\Phi(x, s)$ of the vector field $V$. Since the measure $\mu$ is flow invariant, the right integral in (22) vanishes for any $f$. It is clear that the right hand side of (21) is a linear function of $\xi \in H^{1}(M ; \mathbb{R})$. Hence there exists a unique real homology class $\mathcal{A}_{\mu} \in H_{1}(M ; \mathbb{R})$ which satisfies (21) for all $\xi \in H^{1}(M ; \mathbb{R})$.

Necessary conditions. We consider the flow $\Phi$ as being fixed and we vary the invariant measure $\mu$. As the class $\mathcal{A}_{\mu} \in H_{1}(M ; \mathbb{R})$ depends linearly on $\mu$, the set of asymptotic cycles $\mathcal{A}_{\mu}$ corresponding to all $\Phi$-invariant positive measures $\mu$ forms a convex cone in the vector space $H_{1}(M ; \mathbb{R})$.

Proposition 8. Assume that there exists a Lyapunov 1-form for $(\Phi, Y)$ lying in a cohomology class $\xi \in H^{1}(M ; \mathbb{R})$. Then

$$
\left\langle\xi, \mathcal{A}_{\mu}\right\rangle \leq 0
$$

for any $\Phi$-invariant positive Borel measure $\mu$ on $M$; equality in (23) takes place if and only if the complement of $Y$ has measure zero. Furthermore, the restriction of $\xi$ to $Y$, viewed as a Čech cohomology class

$$
\left.\xi\right|_{Y} \in \check{H}^{1}(Y ; \mathbb{R})
$$

vanishes, $\left.\xi\right|_{Y}=0$.

Proof. Let $\omega$ be a Lyapunov 1-form for $(\Phi, Y)$ lying in the class $\xi$. According to Definition 5, the function $\iota_{V}(\omega)$ is negative on $M-Y$ and vanishes on $Y$. We obtain that the integral

$$
\int_{M} \iota_{V}(\omega) d \mu=\left\langle\xi, \mathcal{A}_{\mu}\right\rangle
$$

is nonpositive.

Assuming $\mu(M-Y)>0$, we find a compact $K \subset M-Y$ with $\mu(K)>0$; This follows from the theorem of Riesz; see, e.g., [30], Theorem 2.3(iv), page 256. There is a constant $\epsilon>0$ such that $\left.\iota_{V}(\omega)\right|_{K} \leq-\epsilon$. Therefore, one has

$$
\int_{M} \iota_{V}(\omega) d \mu \leq-\epsilon \mu(K)<0 .
$$

Hence, the value $\left\langle\xi, \mathcal{A}_{\mu}\right\rangle$ is strictly negative if the measure $\mu$ is not supported in $Y$. 
To prove the second statement, we observe (see [58]) that the Čech cohomology $\check{H}^{1}(Y ; \mathbb{R})$ equals the direct limit of the singular cohomology

$$
\check{H}^{1}(Y ; \mathbb{R})=\lim _{W \supset Y} H^{1}(W ; \mathbb{R}),
$$

where $W$ runs over open neighborhoods of $Y$. It is clear in view of condition $(\Lambda 2)$ that $\left.\xi\right|_{U}=0 \in H^{1}(U ; \mathbb{R})$ (by the de Rham theorem). Hence the result follows.

Chain-recurrent set $R_{\xi}$. Given a flow $\Phi$, our aim is to construct a Lyapunov 1-form $\omega$ for a pair $(\Phi, Y)$ lying in a given cohomology class $\xi \in$ $H^{1}(M ; \mathbb{R})$. A natural candidate for $Y$ is the subset $R_{\xi}=R_{\xi}(\Phi)$ of the chain-recurrent set $R=R(\Phi)$ which was defined in [19]. We briefly recall the definition.

Fix a Riemannian metric on $M$ and denote by $d$ the corresponding distance function. Given any $\delta>0, T>1$, a $(\delta, T)$-chain from $x \in M$ to $y \in M$ is a finite sequence $x_{0}=x, x_{1}, \ldots, x_{N}=y$ of points in $M$ and numbers $t_{1}, \ldots, t_{N} \in \mathbb{R}$ such that $t_{i} \geq T$ and $d\left(x_{i-1} \cdot t_{i}, x_{i}\right)<\delta$ for all $1 \leq i \leq N$. Here we use the notation $\Phi(x, t)=x \cdot t$. The chain recurrent set $R=R(\Phi)$ of the flow $\Phi$ is defined as the set of all points $x \in M$ such that for any $\delta>0$ and $T>1$ there exists a $(\delta, T)$-chain starting and ending at $x$. The chain recurrent set $R$ is closed and invariant under the flow.

Given a cohomology class $\xi \in H^{1}(M ; \mathbb{R})$, there is a natural covering space $p_{\xi}: \tilde{M}_{\xi} \rightarrow M$ associated with $\xi$. A closed loop $\gamma:[0,1] \rightarrow M$ lifts to a closed loop in $\tilde{M}_{\xi}$ if and only if the value of the cohomology class $\xi$ on the homology class $[\gamma] \in H_{1}(M ; \mathbb{Z})$ vanishes, $\langle\xi,[\gamma]\rangle=0$.

The flow $\Phi$ lifts uniquely to a flow $\tilde{\Phi}$ on the covering $\tilde{M}_{\xi}$. Consider the chain recurrent set $R(\tilde{\Phi}) \subset \tilde{M}_{\xi}$ of the lifted flow and denote by $R_{\xi}=p_{\xi}(R(\tilde{\Phi})) \subset$ $M$ its projection onto $M$. The set $R_{\xi}$ is referred to as the chain recurrent set associated to the cohomology class $\xi$. It is clear that $R_{\xi}$ is closed, $\Phi$-invariant, and $R_{\xi} \subset R$.

We denote by $C_{\xi}$ the complement of $R_{\xi}$ in $R$,

$$
C_{\xi}=R-R_{\xi} .
$$

Let us mention the following example illustrating the definition of $R_{\xi}$. Consider a smooth flow on a closed manifold $M$ whose chain recurrent set $R$ consists of finitely many rest points and periodic orbits. Given a cohomology class $\xi \in H^{1}(M ; \mathbb{R})$, the chain recurrent set $R_{\xi}$ is the union of all the rest points and of those periodic orbits whose homology classes $z \in H_{1}(M ; \mathbb{Z})$ satisfy $\langle\xi, z\rangle=0$.

In general, any fixed point of the flow belongs to $R_{\xi}$. The points of a periodic orbit belong to $R_{\xi}$ if the homology class $z \in H_{1}(X ; \mathbb{Z})$ of this orbit satisfies $\langle\xi, z\rangle=0$.

It may happen that the points of a periodic orbit belong to $R_{\xi}$ although $\langle\xi, z\rangle \neq 0$ for the homology class $z$ of the orbit. This possibility is illustrated 
by the following example; cf. [19]. Consider the flow on the planar ring

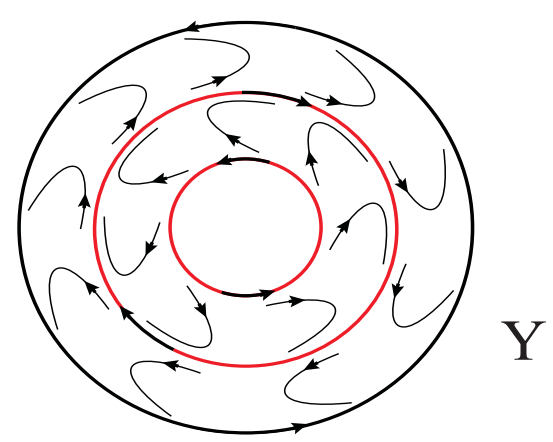

Figure 1. The flow on the planar ring $Y$.

$Y \subset \mathbb{C}$ shown in Figure 1. In the polar coordinates $(r, \phi)$ the ring $Y$ is given by the inequalities $1 \leq r \leq 3$ and the flow is given by the differential equations

$$
\dot{r}=(r-1)^{2}(r-3)^{2}(r-5)^{2}, \quad \dot{\phi}=\sin \left(r \cdot \frac{\pi}{2}\right) .
$$

Let $C_{k}$, where $k=1,2,3$, denote the circle $r=2 k-1$. The circles $C_{1}, C_{2}, C_{3}$ are invariant under the flow. The motion along the circles $C_{1}$ and $C_{3}$ has constant angular velocity 1 . Identifying any point $(r, \phi) \in C_{1}$ with $(5 r, \phi) \in$ $C_{3}$, we obtain a torus $X=Y / \simeq$ and a flow $\Phi: X \times \mathbb{R} \rightarrow X$. The images of the circles $C_{1}, C_{2}, C_{3} \subset Y$ represent two circles $C_{1}^{\prime}=C_{3}^{\prime}$ and $C_{2}^{\prime}$ on the torus $X$. Let $\xi \in H^{1}(X ; \mathbb{R})$ be a nonzero cohomology class which is the pullback of a cohomology class of $Y$. One verifies that in this example the set $R_{\xi}(\Phi)$ coincides with the whole torus $X$. In particular, $R_{\xi}(\Phi)$ contains the periodic orbits $C_{1}^{\prime}=C_{3}^{\prime}$ and $C_{2}^{\prime}$ although clearly $\left\langle\xi,\left[C_{k}^{\prime}\right]\right\rangle \neq 0$.

A different definition of $R_{\xi}$ which does not use the covering space $\tilde{M}_{\xi}$ can be found in [19].

To state the main result of this section, we need the following notion. A $(\delta, T)$-cycle of the flow $\Phi$ is defined as a pair $(x, t)$, where $x \in M$ and $t>T$ such that $d(x, x \cdot t)<\delta$. If $\delta$ is small enough, then any $(\delta, T)$-cycle determines in a canonical way a unique homology class $z \in H_{1}(M ; \mathbb{Z})$ which is represented by the flow trajectory from $x$ to $x \cdot t$ followed by a "short" arc connecting $x \cdot t$ with $x$. See [19] for more details.

Theorem 5 (Farber, Kappeler, Latschev, Zehnder [20]). Let $V$ be a smooth vector field on a smooth closed manifold $M$. Denote by $\Phi: M \times \mathbb{R} \rightarrow M$ the flow generated by $V$. Let $\xi \in H^{1}(M ; \mathbb{R})$ be a cohomology class such that the restriction $\left.\xi\right|_{R_{\xi}}$, viewed as a Čech cohomology class $\left.\xi\right|_{R_{\xi}} \in \check{H}^{1}\left(R_{\xi} ; \mathbb{R}\right)$, vanishes. Then the following properties of $\xi$ are equivalent: 


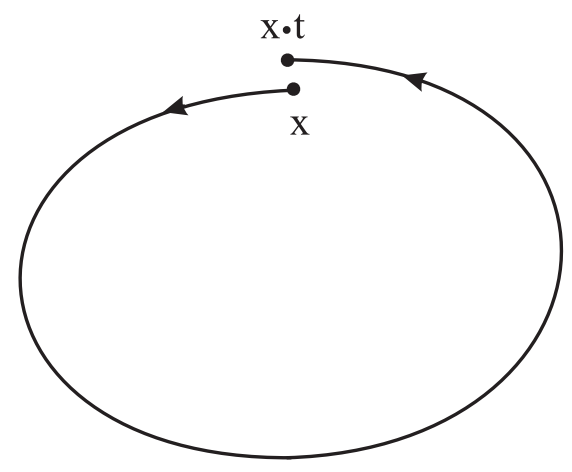

Figure 2. $(\delta, T)$-cycle.

(I) There exists a smooth Lyapunov 1-form for $\left(\Phi, R_{\xi}\right)$ in the cohomology class $\xi$ and the subset $C_{\xi}$ is closed.

(II) For any Riemannian metric on $M$ there exist $\delta>0$ and $T>1$ such that the homology class $z \in H_{1}(M ; \mathbb{Z})$ associated with an arbitrary $(\delta, T)$-cycle $(x, t)$ of the flow, with $x \in C_{\xi}$, satisfies $\langle\xi, z\rangle \leq-1$.

(III) The subset $C_{\xi}$ is closed and there exists a constant $\eta>0$ such that for any $\Phi$-invariant positive Borel measure $\mu$ on $M$ the asymptotic cycle $\mathcal{A}_{\mu}=\mathcal{A}_{\mu}(\Phi) \in H_{1}(M ; \mathbb{R})$ satisfies

$$
\left\langle\xi, \mathcal{A}_{\mu}\right\rangle \leq-\eta \cdot \mu\left(C_{\xi}\right) .
$$

(IV) The subset $C_{\xi}$ is closed and for any $\Phi$-invariant positive Borel measure $\mu$ on $X$ with $\mu\left(C_{\xi}\right)>0$, the asymptotic cycle $\mathcal{A}_{\mu}=\mathcal{A}_{\mu}(\Phi) \in H_{1}(M ; \mathbb{R})$ satisfies

$$
\left\langle\xi, \mathcal{A}_{\mu}\right\rangle<0
$$

Condition (24) can be reformulated using the notion of a quasi-regular point. Recall that $x \in X$ is a quasi-regular point of the flow $\Phi: X \times \mathbb{R} \rightarrow X$ if for any continuous function $f: X \rightarrow \mathbb{R}$ the limit

$$
\lim _{t \rightarrow \infty} \frac{1}{t} \int_{0}^{t} f(x \cdot s) d s
$$

exists. It follows from the ergodic theorem that the subset $Q \subset X$ of all quasi-regular points has full measure with respect to any $\Phi$-invariant positive Borel measure on $X$. From the Riesz representation theorem (see, e.g., [50], page 256) one deduces that for any quasi-regular point $x \in Q$ there exists a unique positive flow-invariant Borel measure $\mu_{x}$ with $\mu_{x}(X)=1$ satisfying

$$
\lim _{t \rightarrow \infty} \frac{1}{t} \int_{0}^{t} f(x \cdot s) d s=\int_{X} f d \mu_{x}
$$


for any continuous function $f$. We use below the well-known fact that any positive, $\Phi$-invariant Borel measure $\mu$ with $\mu(X)=1$ belongs to the weak* closure of the convex hull of the set of measures $\mu_{x}, x \in Q$.

If the subset $C_{\xi} \subset X$ is closed, and hence compact, one can apply the abovementioned facts to the restriction of the flow to $C_{\xi}$. Let $\omega$ be an arbitrary smooth closed 1-form lying in the cohomology class $\xi$. For any quasi-regular point $x \in C_{\xi}$ of the flow $\left.\Phi\right|_{C_{\xi}}$ one has

$$
\begin{aligned}
\lim _{t \rightarrow \infty} \frac{1}{t} \int_{x}^{x \cdot t} \omega & =\lim _{t \rightarrow \infty} \frac{1}{t} \int_{0}^{t} \iota_{V}(\omega)(x \cdot s) d s \\
& =\int_{M} \iota_{V}(\omega) d \mu_{x}=\left\langle\xi, \mathcal{A}_{\mu_{x}}\right\rangle .
\end{aligned}
$$

We therefore conclude that condition (III) is equivalent to:

$\left(\mathrm{III}^{\prime}\right)$ The subset $C_{\xi}$ is closed and there exists a constant $\eta>0$ such that for any quasi-regular point $x \in C_{\xi}$,

$$
\lim _{t \rightarrow \infty} \frac{1}{t} \int_{x}^{x \cdot t} \omega \leq-\eta
$$

where $\omega$ is an arbitrary closed 1-form in the class $\xi$.

The value of the limit (29) is independent of the choice of a closed 1-form $\omega$; the only requirement is that $\omega$ lies in the cohomology class $\xi$.

In the special case $\xi=0$ the set $C_{\xi}$ is empty and $R=R_{\xi}$. The above statement then reduces to the following well-known theorem of C. Conley (see [4] and [54, Theorem 3.14):

Theorem 6. (C. Conley) Let $V$ be a smooth vector field on a smooth closed manifold $M$. Denote by $\Phi: M \times \mathbb{R} \rightarrow M$ the flow generated by $V$ and by $R$ the chain recurrent set of $\Phi$. Then there exists a smooth Lyapunov function $L: M \rightarrow \mathbb{R}$ for $(\Phi, R)$. This means that $V(L)<0$ on $M-R$ and $d L=0$ pointwise on $R$.

A proof of Theorem 5 can be found in [20].

We also refer to [19] where continuous Lyapunov 1-forms are studied. The paper [19] also contains a discussion comparing the results about the existence of Lyapunov 1-forms with the theorems of D. Fried [27] about the existence of cross sections for flows.

Finally we describe a class of examples of flows for which there exists a cohomology class $\xi$ satisfying all the conditions of Theorem 5 .

Let $M$ be a closed smooth manifold with a smooth vector field $v$. Let $\Psi: M \times \mathbb{R} \rightarrow M$ be the flow of $v$. Assume that the chain recurrent set $R(\Psi)$ is a union of two disjoint closed sets $R(\Psi)=R_{1} \cup R_{2}$, where $R_{1} \cap R_{2}=\emptyset$. Out of this data we construct a flow $\Phi$ on

$$
X=M \times S^{1}
$$


such that $R_{\xi}(\Phi)=R_{1} \times S^{0}, C_{\xi}=R_{2} \times S^{1}$. Here $\xi \in H^{1}(X ; \mathbb{Z})$ denotes the de Rham cohomology class of the 1 -form $-d \theta$ where $\theta \in[0,2 \pi]$ denotes the angle coordinate on the circle $S^{1} ; S^{0} \subset S^{1}$ is a two-point set.

We will need two vector fields $w_{1}$ and $w_{2}$ on $S^{1}, w_{1}=\cos (\theta) \cdot \frac{\partial}{\partial \theta}$ and $w_{2}=\frac{\partial}{\partial \theta}$. The field $w_{1}$ has two zeros $\left\{p_{1}, p_{2}\right\}=S^{0} \subset S^{1}$ corresponding to the angles $\theta=\pi / 2$ and $\theta=3 \pi / 2$.

Let $f_{i}: M \rightarrow[0,1]$, where $i=1,2$, be two smooth functions having disjoint supports and satisfying $\left.f_{1}\right|_{R_{1}}=1,\left.f_{2}\right|_{R_{2}}=1$.

Consider the flow $\Phi: X \times \mathbb{R} \rightarrow X$ determined by the vector field

$$
V=v+f_{1} w_{1}+f_{2} w_{2}
$$

Any trajectory of $V$ has the form $(\gamma(t), \theta(t))$, where $\dot{\gamma}(t)=v(\gamma(t))$, i.e., $\gamma(t)$ is a trajectory of $v$. It follows that the chain recurrent set of $V$ is contained in $R(\Psi) \times S^{1}$. Over $R_{1}$ we have the vertical vector field $w_{1}$ along the circle which has two points $S^{0} \subset S^{1}$ as its chain recurrent set. Over $R_{2}$ we have the vertical vector field $w_{2}$ which has all of $S^{1}$ as the chain recurrent set. We see that $R_{1} \times S^{0}=R_{\xi}(\Phi), R_{2} \times S^{1}=C_{\xi}$. Hence

$$
\left.\xi\right|_{R_{\xi}}=0
$$

and $C_{\xi}$ is closed. One easily checks that condition (III) of Theorem 5 (and hence the other conditions as well) is satisfied.

Further examples can be found in $\S 7$ of [19].

An approach to study dynamical systems using cocycles instead of Lyapunov closed 1-forms was suggested by H. Fan and J. Jost [9], [10].

\section{Notions of CATEGory With RespeCt to A COHOMOlOGY ClASS}

In this section we describe several generalizations of the classical notion of Lusternik - Schnirelmann category which reflect interesting dynamical properties of flows on manifolds. These new notions depend on a choice of a cohomology class $\xi \in H^{1}(X ; \mathbb{R})$ and are all equal to the classical invariant $\operatorname{cat}(X)$ in the case $\xi=0$.

5.1. Movable subsets. First we define the notion of movability of a subset with respect to a closed one-form.

Definition 6. Let $\omega$ be a closed one-form on a topological space $X$. A subset $A \subset X$ is called $N$-movable with respect to $\omega$ (where $N$ is an integer), if there is a homotopy $H: A \times[0,1] \rightarrow X$ such that $H(a, 0)=a$ for all $a \in A$, and

$$
\int_{a}^{H_{1}(a)} \omega \leq-N
$$

for all $a \in A$. 
Note that the latter inequlaity

Below we shall think of the form $\omega$ being fixed and of integer $N$ being large, or tending to infinity. This may happen only in the case when the cohomology class $\xi=[\omega] \in H^{1}(X ; \mathbb{R})$ is nonzero. Indeed, it is clear that in the case of an exact form $\omega=d f$, a nonempty subset $A \subset X$ can be $N$-movable with respect for $\omega$ only for $N \leq \max f-\min f$.

Any subset $A \subset X$ such that the inclusion $A \rightarrow X$ is null-homotopic is $N$ movable with respect to any closed 1-forms $\omega$ assuming that the cohomology class $\xi=[\omega] \neq 0 \in H^{1}(X ; \mathbb{R})$ is nonzero.

As another example consider the following situation. Assume that $X$ is a closed smooth manifold and $\omega$ is a smooth closed one-form on $X$. If $\omega$ has no zeros then any subset $A \subset X$ is $N$-movable with respect to $\omega$ for any integer $N$. In this case a homotopy $H: A \times I \rightarrow X$ is given by the gradient flow (appropriately scaled) of $\omega$ with respect to a Riemannian metric on $X$. In general there are topological obstructions to movability of subsets which are captured by topological invariants of Lusternik - Schnirelmann type described below.

5.2. Various notions of category. Here is the first version of the notion of a category $\operatorname{cat}(X, \xi)$ with respect to a cohomology class. The definition given below is equivalent to the original definition of [15], see [17].

Definition 7. Let $X$ be a finite CW-complex and $\xi \in H^{1}(X ; \mathbb{R})$. Fix a closed 1 -form $\omega$ representing $\xi$. Then the number

$$
\operatorname{cat}(X, \xi)
$$

is defined the minimal integer $k$ such that for every $N>0$ there exists a closed subset $A \subset X$ which is $N$-movable with respect to $\omega$ and such that $\operatorname{cat}_{X}(X-A) \leq k$.

Recall that for $A \subset X$, $\operatorname{cat}_{X}(A)$ is the minimal integer $k$ such that $A$ can be covered by $k$ open subsets of $X$ each of which is null-homotopic in $X$.

Note that the number $\operatorname{cat}(X, \xi)$ does not depend on the choice of a closed one-form $\omega$ and may depend only on the cohomology class $\xi=[\omega] \epsilon$ $H^{1}(X, ; \mathbb{R})$. Indeed, if $\omega^{\prime}$ is another closed 1-form lying in the same cohomology $\xi=\left[\omega^{\prime}\right]$ then $\omega^{\prime}=\omega+d f$ for some continuous function $f: X \rightarrow \mathbb{R}$. Then for any path $\gamma:[a, b] \rightarrow X$ one has

$$
\int_{\gamma} \omega^{\prime}=\int_{\gamma} \omega+f(\gamma(b))-f(\gamma(a))
$$

Since $X$ is compact we see that there exists a constant $C>0$ such that for all paths $\gamma$ in $X$ one has

$$
\left|\int_{\gamma} \omega^{\prime}-\int_{\gamma} \omega\right|<C
$$


Hence any subset $A \subset X$ which is $N$-movable with respect to $\omega$ is $(N-C)$ movable with respect to $\omega^{\prime}$.

It may happen that

$$
\operatorname{cat}(X, \xi) \neq \operatorname{cat}(X,-\xi),
$$

see Example 10.10 in [18]. Therefore it makes sense to introduce (as was suggested first in [60]) a symmetric version $\operatorname{cat}_{s}(X, \xi)$ of $\operatorname{cat}(X, \xi)$ which suit better dynamics applications:

Definition 8. Let $X$ be a finite $\mathrm{CW}$-complex and $\xi \in H^{1}(X ; \mathbb{R})$. Fix a closed 1 -form $\omega$ representing $\xi$. Then the number

$$
\operatorname{cat}_{s}(X, \xi)
$$

is defined the minimal integer $k$ such that for every $N>0$ there exists a closed subset $A \subset X$ which is $N$-movable with respect to both $\omega$ and $-\omega$ and such that $\operatorname{cat}_{X}(X-A) \leq k$.

Clearly, one has

$$
\operatorname{cat}_{s}(X, \xi)=\operatorname{cat}_{s}(X,-\xi) \geq \max \{\operatorname{cat}(X, \xi), \operatorname{cat}(X,-\xi)\} .
$$

Reversing the quantifiers in Definition 7 gives a slightly different notion $\operatorname{cat}^{1}(X, \xi)$ which was initially introduced in [17]:

Definition 9. Let $X$ be a finite $\mathrm{CW}$-complex and $\xi \in H^{1}(X ; \mathbb{R})$. Fix a closed 1 -form $\omega$ representing $\xi$. Then the number $\operatorname{cat}^{1}(X, \xi)$ is defined as the minimal integer $k$ such that there exists a closed subset $A \subset X$, which is $N$ movable with respect to $\omega$ for every $N>0$, and such that cat ${ }_{X}(X-A) \leq k$.

The invariant $\operatorname{cat}^{1}(X, \xi)$ also has a symmetric modification $\operatorname{cat}_{s}^{1}(X, \xi)$. The latter is defined similarly to $\operatorname{cat}^{1}(X, \xi)$ with the only difference that in Definition 9 one requires that the closed subset $A \subset X$ is $N$-movable with respect to both one-form $\omega$ and $-\omega$.

Yet another invariant similar in spirit is given by the following definition:

Definition 10. Let $X$ be a finite CW-complex and $\xi \in H^{1}(X ; \mathbb{R})$. Fix a closed 1 -form $\omega$ representing $\xi$. Then $\operatorname{Cat}(X, \xi)$ is defined as the minimal integer $k$ such that there exists an open subset $U \subset X$ satisfying

(a) $\operatorname{cat}_{X}(X-U) \leq k$;

(b) for some homotopy $h: U \times[0, \infty) \rightarrow X$ one has

$$
h(x, 0)=x \text { and } \lim _{t \rightarrow \infty} \int_{x}^{h_{t}(x)} \omega=-\infty
$$

for any point $x \in U$;

(c) the limit in (b) is uniform in $x \in U$.

The symbol $\int_{x}^{h_{t}(x)} \omega$ denotes the integral $\int_{\gamma} \omega$ where the path $\gamma:[0, t] \rightarrow X$ is given by $\gamma(\tau)=h_{\tau}(x)$. 
Remark 1. The definition of $\operatorname{Cat}(X, \xi)$ given above differs from the original definition suggested in [16; the latter was symmetric and stronger in some examples.

Independence of $\operatorname{cat}^{1}(X, \xi)$ and $\operatorname{Cat}(X, \xi)$ of the choice of closed one-form $\omega$ with $[\omega]=\xi$ follows similarly to the argument given above for the case of $\operatorname{cat}(X, \xi)$.

The role the invariants $\operatorname{cat}(X, \xi), \operatorname{cat}^{1}(X, \xi)$ and $\operatorname{Cat}(X, \xi)$ play in dynamics will be discussed in the following sections.

Lemma 9. In the case of the trivial cohomology class $\xi=0 \in H^{1}(X ; \mathbb{R})$ the numbers

$$
\operatorname{cat}(X, \xi)=\operatorname{cat}^{1}(X, \xi)=\operatorname{Cat}(X, \xi)=\operatorname{cat}(X)
$$

coincide with the classical Lusternik - Schnirelmann category cat $(X)$.

Proof. We will give the proof for $\operatorname{cat}(X, \xi)$. If $\xi=0$ we may take $\omega=0$ and hence any subset $A \subset X$ which is $N$-movable with respect to $\omega$ is empty assuming that $N>0$. Hence we obtain that $\operatorname{cat}(X, \xi)=\operatorname{cat}_{X}(X)=$ $\operatorname{cat}(X)$.

Example 1. Suppose that $p: X \rightarrow S^{1}$ is a locally trivial fibration and $\xi=p^{*}(\eta)$ where $\eta \in H^{1}\left(S^{1} ; \mathbb{R}\right), \eta \neq 0$. Then

$$
\operatorname{cat}(X, \xi)=\operatorname{cat}^{1}(X, \xi)=\operatorname{Cat}(X, \xi)=0
$$

(see [18]). Note that cat $(X)$ can be arbitrarily large in this example. Hence the new "cats" are not always equal the classical cat $(X)$.

Comparing the definitions one trivially has

$$
\operatorname{cat}(X, \xi) \leq \operatorname{cat}^{1}(X, \xi) \leq \operatorname{Cat}(X, \xi) .
$$

We will show later that $\operatorname{cat}(X, \xi)$ may be significantly smaller than $\operatorname{cat}^{1}(X, \xi)$. At the moment we do not have examples when $\operatorname{cat}^{1}(X, \xi)<\operatorname{Cat}(X, \xi)$ but we believe that such examples exist.

Lemma 10. Suppose that $\xi \neq 0$ and $X$ is connected. Then

$$
\operatorname{Cat}(X, \xi) \leq \operatorname{cat}(X)-1
$$

The proof of this Lemma can be found in [18].

As another example consider a bouquet $X=Y \vee S^{1}$, where $Y$ is a finite polyhedron, and assume that the class $\xi \in H^{1}(X, \mathbb{R})$ satisfies $\left.\xi\right|_{Y}=0$ and $\left.\xi\right|_{S^{1}} \neq 0$. One can show (see [18]) that then

$$
\operatorname{cat}(X, \xi)=\operatorname{cat}(Y)-1 \text {. }
$$




\subsection{Homotopy invariance.}

Proposition 11. Let $f: Y \rightarrow X$ be a homotopy equivalence between finite connected $C W$-complexes, and $\xi \in H^{1}(X ; \mathbb{R})$. Then

$$
\operatorname{cat}(X, \xi)=\operatorname{cat}\left(Y, f^{*} \xi\right) .
$$

This statement remains true if one replaces cat by cat $^{1}$ or Cat.

Proof. Let $\omega$ be a closed 1-form on $X$ representing $\xi$. Let $g: X \rightarrow Y$ and $K: X \times[0,1] \rightarrow X$ satisfy $K: \operatorname{id}_{X} \simeq f g$. Because of compactness of $X$, there exists a constant $C>0$ such that

$$
\left|\int_{\alpha_{x}} \omega\right| \leq C
$$

for all $x \in X$, where $\alpha_{x}(t)=K(x, t)$. Assume that a subset $A \subset Y$ is $(N+C)$-movable with respect to the closed one-form $\omega^{\prime}=f^{*} \omega$ on $Y$. Let $H: A \times[0,1] \rightarrow Y$ be a homotopy such that $H(a, 0)=a$ and $\int_{\gamma_{a}} \omega^{\prime} \geq N+C$ for all $a \in A$ where $\gamma_{a}(t)=H(a, 1-t)$. Then the set $g^{-1}(A) \subset X$ is $N$-movable with respect to $\omega$. Indeed, one may define a homotopy $h_{t}$ : $g^{-1}(A) \rightarrow X$ by

$$
h_{t}(x)= \begin{cases}K(x, 2 t) & \text { for } 0 \leq t \leq 1 / 2 \\ f(H(g(x), 2 t-1)) & \text { for } 1 / 2 \leq t \leq 1\end{cases}
$$

and for $x \in g^{-1}(A)$ one has $\int_{\beta_{x}} \omega \geq N$ where $\beta_{x}(t)=h_{1-t}(x)$.

If $\operatorname{cat}\left(Y, f^{*} \xi\right) \leq k$ then for any $N$ there exists a closed subset $A \subset Y$ which is $(N+C)$-movable with respect to $\omega^{\prime}=f^{*} \omega$ and $\operatorname{such}$ that $\operatorname{cat}_{Y}(Y-A) \leq k$. Then $g^{-1}(A) \subset X$ is $N$-movable with respect to $\omega$ and, as is well-known, one has $\operatorname{cat}_{X}\left(X-g^{-1}(A)\right) \leq k$. This proves that $\operatorname{cat}(X, \xi) \leq \operatorname{cat}\left(Y, f^{*} \xi\right)$.

The inverse inequality follows similarly if $g f \simeq \operatorname{id}_{Y}$. The arguments for $\operatorname{cat}^{1}(X, \xi)$ and $\operatorname{Cat}(X, \xi)$ are similar.

5.4. Spaces of category zero. Here we collect some simple observations about spaces of category zero. More information can be found in [60], §3.

Lemma 12. Let $X$ be a finite $C W$ complex and $\xi \in H^{1}(X ; \mathbb{R})$. The following properties are equivalent:

(a) $\operatorname{cat}(X, \xi)=0$.

(b) $\operatorname{cat}^{1}(X, \xi)=0$.

(c) $\operatorname{Cat}(X, \xi)=0$.

(d) There exists a continuous closed 1 -form $\omega$ on $X$ representing $\xi$ (in the sense of subsection (3.3) and a homotopy $h_{t}: X \rightarrow X$, where $t \in[0,1]$, such that for any point $x \in X$ one has

$$
\int_{x}^{h_{1}(x)} \omega<0 .
$$

In (33) the integral is calculated along the curve $t \mapsto h_{t}(x), t \in[0,1]$. 
(e) For any continuous closed 1 -form $\omega$ on $X$ representing $\xi$ there exists a homotopy $h_{t}: X \rightarrow X$, where $t \in[0,1]$, such that for any point $x \in X$ inequality (33) holds.

Proof. By Definition 7, $\operatorname{cat}(X, \xi)=0$ means that the whole space $X$ is $N$-movable for any $N>0$, i.e. given $N>0$, there exists a homotopy $H_{t}: X \rightarrow X$, where $t \in[0,1]$, such that $H_{0}(x)=x$ and

$$
\int_{x}^{H_{1}(x)} \omega<-N
$$

for any $x \in X$. Hence, (a) implies (d).

Conversely, given property (d), using compactness of $X$ we find $\epsilon>0$ such that (33) can be replaced by $\int_{x}^{h_{1}(x)} \omega<-\epsilon$. Now, one may iterate this deformation as follows. The $k$-th iteration is a homotopy $H_{t}^{k}: X \rightarrow X$, where $t \in[0,1]$, defined as follows. Denote by $h_{1}^{(i)}: X \rightarrow X$ the $i$-fold composition $h_{1}^{(i)}=h_{1} \circ h_{1} \circ \cdots \circ h_{1}$ (i times). Then for $t \in[i / k,(i+1) / k]$ one has

$$
H_{t}^{k}(x)=h_{k t-i}\left(h_{1}^{(i)}(x)\right) .
$$

If for any $x \in X$ one has $\int_{x}^{h_{1}(x)} \omega<-\epsilon$ then for the $k$-th iteration we obtain $\int_{x}^{H_{1}^{k}(x)} \omega<-k \epsilon$ and (d) follows assuming that $k>N / \epsilon$. This shows equivalence between (a) and (d).

(e) $\Longrightarrow(\mathrm{d})$ is obvious. Now suppose that (d) holds for $\omega$ and let $\omega_{1}$ be another continuous closed one-form lying in the same cohomology class, i.e. $\omega_{1}=\omega+d f$ where $f: X \rightarrow \mathbb{R}$ is continuous. Using compactness of $X$ we may find $C$ such that for any path $\gamma:[0,1] \rightarrow X$ one has $\left|\int_{\gamma} d f\right|<C$. Fix $N>C$ and apply equivalence between (a) and (c) to find a homotopy $h_{t}: X \rightarrow X$ with $\int_{x}^{h_{1}(x)} \omega<-N$. Then one has $\int_{x}^{h_{1}(x)} \omega_{1}<0$, i.e. (e) holds. It is obvious that (b) $\Longrightarrow(a)$. Hence we are left to show that (a) implies (b). Given (b) fix a deformation as described in (c). Let $C>0$ be such that for any $x \in X$ and for any $t \in[0,1]$ one has $\int_{1}^{h_{t}(x)} \omega<C$. Then for any iteration $H_{t}^{k}: X \rightarrow X$ (see above) one has $\int_{x}^{H_{t}^{k}(x)} \omega<C$ and the result follows.

In the case when $X$ is a closed smooth manifold a deformation as appearing in (b) can be constructed as the flow generated by a vector field $v$ on $X$ satisfying $\omega(v)<0$.

The remark of the previous paragraph explains why the following statement can be viewed as an analogue of the classical Euler - Poincaré theorem:

Theorem 7. $\operatorname{cat}(X, \xi)=0$ implies $\chi(X)=0$.

Proof. Suppose that $\operatorname{cat}(X, \xi)=0$. Then $\xi \neq 0$, i.e. the rank $r$ of class $\xi$ is positive, $r>0$. By Lemma 20 below there exists $\xi$-transcendental bundle 
$L \in \mathcal{V}_{\xi}=\left(\mathbb{C}^{*}\right)^{r}$. If $H^{q}(X ; L) \neq 0$ for some $q$ then one may apply Theorem 19 below with $k=0$ obtaining $\operatorname{cat}(X, \xi)>0$ and contradicting our hypothesis. Hence $H^{q}(X ; L)=0$ for all $q$ which implies

$$
\chi(X)=\sum_{q}(-1)^{q} \operatorname{dim} H^{q}(X ; L)=0 .
$$

Pairs $(X, \xi)$ with $\operatorname{cat}(X, \xi)=0$ form an "ideal" in the following sense:

Lemma 13. Let $X_{1}$ and $X_{2}$ be finite cell complexes and $\xi_{1} \in H^{1}\left(X_{1} ; \mathbb{R}\right)$, $\xi_{2} \in H^{1}\left(X_{2} ; \mathbb{R}\right)$. If $\operatorname{cat}\left(X_{1}, \xi_{1}\right)=0$ then $\operatorname{cat}\left(X_{1} \times X_{2}, \xi\right)=0$ where $\xi=$ $\xi_{1} \times 1+1 \times \xi_{2} \in H^{1}\left(X_{1} \times X_{2} ; \mathbb{R}\right)$.

Proof. The statement follows directly by applying the definitions.

\section{Focusing EFFeCT}

In this section we start surveying applications of the invariant cat $(X, \xi)$ in dynamics. We describe the focusing effect discovered in [15], see also [18].

The nature of the Lusternik-Schnirelmann type theory for closed 1-forms is very much distinct from both the classical Lusternik-Schnirelmann theory for functions and from the Novikov theory. As we know, in any nonzero cohomology class $\xi \in H^{1}(M ; \mathbb{Z}), \xi \neq 0$, one may always find a representing closed 1-form having at most one (highly degenerate) zero. Hence, quantitative estimates on the number of zeros may be obtained only under some additional assumptions. It turns out that these additional assumptions can be expressed in terms of some interesting dynamical properties of gradientlike vector fields for a given closed 1-form. This makes the new theory potentially very useful for dynamics.

Let $v$ be a smooth vector field on a closed smooth manifold $M$. Recall that a homoclinic orbit of $v$ is defined as an integral trajectory $\gamma(t)$,

$$
\dot{\gamma}(t)=v(\gamma(t)), \quad t \in \mathbb{R}
$$

such that both limits $\lim _{t \rightarrow+\infty} \gamma(t)$ and $\lim _{t \rightarrow-\infty} \gamma(t)$ exist and are equal

$$
\lim _{t \rightarrow+\infty} \gamma(t)=\lim _{t \rightarrow-\infty} \gamma(t) .
$$

More generally, a homoclinic cycle of length $n$ is a sequence of integral trajectories $\gamma_{1}(t), \gamma_{2}(t), \ldots, \gamma_{n}(t)$ of $v$ such that

$$
\lim _{t \rightarrow+\infty} \gamma_{i}(t)=p_{i+1}=\lim _{t \rightarrow-\infty} \gamma_{i+1}(t)
$$

for $i=1, \ldots, n-1$ and

$$
\lim _{t \rightarrow+\infty} \gamma_{n}(t)=p_{1}=\lim _{t \rightarrow-\infty} \gamma_{1}(t) .
$$

Homoclinic orbits were discovered by H. Poincaré and were studied by S. Smale. In the mathematical literature there are many results about existence 

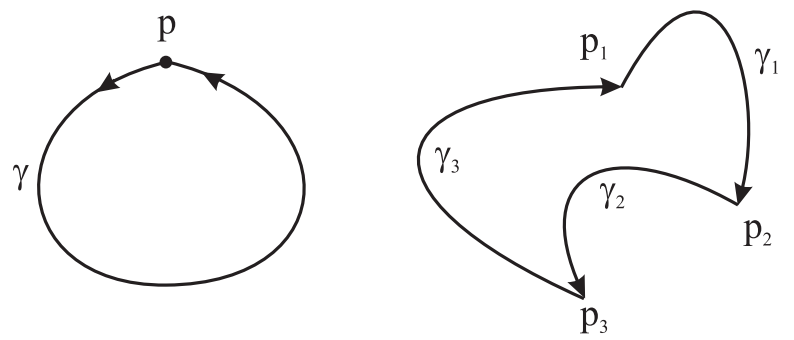

FiguRE 3. Homoclinic orbit (left) and homoclinic cycle (right).

of homoclinic orbits in Hamiltonian systems. For obvious reasons homoclinic orbits cannot exist in gradient systems for functions corresponding to the classical Lusternik-Schnirelmann theory.

One of the main theorems of this section (Theorem 8, originally established in [15]) states that any smooth closed 1-form $\omega$ on a smooth closed manifold $X$ must have at least $\operatorname{cat}(X, \xi)$ geometrically distinct zeros (where $\xi=[\omega] \in$ $H^{1}(X ; \mathbb{R})$ denotes the cohomology class of $\left.\omega\right)$ assuming that $\omega$ admits a gradient-like vector field with no homoclinic cycles.

Viewed differently, the main result of the section claims that any gradientlike vector field of a closed 1-form $\omega$ has a homoclinic cycle if the number of zeros of $\omega$ is less than $\operatorname{cat}(M, \xi)$.

Definition 11. Let $M$ be a smooth closed manifold and let $\omega$ be a smooth closed 1-form on $M$ with the set of zeros

$$
Y_{\omega}=\left\{p \in M ; \omega_{p}=0\right\} .
$$

We say that a smooth vector field $v$ on $M$ is a gradient-like vector field for $\omega$ if $\omega$ is a Lyapunov 1 -form for the pair $\left(\Phi, Y_{\omega}\right)$ where $\Phi$ is the flow on $M$ generated by the field $-v$, see Definition 5 .

Note that this implies that

$$
\omega(v)>0
$$

on $M-Y_{\omega}$ and $Y_{\omega}$ is invariant with respect to the flow generated by $v$.

In the special case when the set of zeros $Y_{\omega}$ is finite, a vector field $v$ is a gradient-like vector field for $\omega$ iff the sets of zeros of $\omega$ and of $v$ coincide and the inequality (36) holds on $M-Y_{\omega}$.

Theorem 8 (Farber [15]). Let $\omega$ be a smooth closed 1-form on a closed smooth manifold $M$. If $\omega$ admits a gradient-like vector field $v$ with no homoclinic cycles then $\omega$ has at least $\operatorname{cat}(M,[\omega])$ geometrically distinct zeros.

Here $[\omega] \in H^{1}(M ; \mathbb{R})$ denotes the cohomology class of $\omega$.

Next we give a reformulation of the above theorem: 
Theorem 9. If the number of zeros of a smooth closed 1-form $\omega$ is less than $\operatorname{cat}(M,[\omega])$, then any gradient-like vector field $v$ for $\omega$ has a homoclinic cycle.

Note that the definition of gradient-like vector field in this paper is slightly more general than the one used in [15], [18].

A slightly more informative statement was proven in [18]:

Theorem 10 (Farber [18], Theorem 10.16). Let $\omega$ be a smooth closed 1-form on a closed manifold $M$ having less than cat $(M, \xi)$ zeros, where $\xi=[\omega] \in$ $H^{1}(M ; \mathbb{R})$. Then there exists an integer $N>0$ such that any gradient-like vector field $v$ for $\omega$ has a homoclinic cycle $\gamma_{1}, \ldots, \gamma_{n}$ satisfying

$$
\sum_{i=1}^{n} \int_{\gamma_{i}} \omega \leq N .
$$

One may combine these results with Theorem 4 which guarantees existence of closed 1-forms with at most one zero in any nonzero integral cohomology class. This shows that there always exist homoclinic cycles which cannot be destroyed while perturbing the gradient-like vector field!

This "focusing effect" starts when the number of zeros of a closed 1-form becomes less than the number $\operatorname{cat}(M, \xi)$. It is a new phenomenon, not occurring in Novikov theory. Indeed, if we assume that the zeros of $\omega$ are all Morse type, then (by the Kupka-Smale Theorem [57]) it is always possible to find a gradient-like vector field $v$ for $\omega$ such that any integral trajectory connecting two zeros comes out of a zero with higher Morse index and goes into a zero with lower Morse index; such a vector field $v$ has no homoclinic cycles.

As an illustration we will state here the following result which is a consequence of Theorem 9 and depends on a calculation of $\operatorname{cat}(X, \xi)$ in the case when $X$ is a product of surfaces, see Theorem 25 below.

Theorem 11. Let $M^{2 k}$ denote the product $\Sigma_{1} \times \Sigma_{2} \times \cdots \times \Sigma_{k}$ where each $\Sigma_{i}$ is a closed orientable surface of genus $g_{i}>1$. For a cohomology class $\xi=[\omega] \in H^{1}(X ; \mathbb{R})$ we denote by $r(\xi)$ the number of indices $i \in\{1, \ldots, k\}$ such that $\xi \mid \Sigma_{i}=0$. Let $\omega$ be a smooth closed 1-form on $M$ lying in the cohomology class $\xi$ and having at most $2 r(\xi)$ zeros. Then any gradient-like vector field for $\omega$ has a homoclinic cycle.

Theorem 9 was generalized by J. Latschev [31] who studied a more general case of closed 1-forms having infinitely many zeros. The result of Latschev gives a "comparison" between the topology of the set of zeros of $\omega$ and the invariant $\operatorname{cat}(M,[\omega])$. We prove below in $\oint 8$ of this paper the following strengthening of the main Theorem of [31] and of Theorem 10. 
Theorem 12. Let $\omega$ be a smooth closed 1-form on a smooth closed manifold $M$. Assume that the set of zeros $Y=\left\{p \in M ; \omega_{p}=0\right\}$ admits a neighbourhood $Y \subset U \subset M$ such that $\omega \mid U$ is exact and $Y$ has finitely many connected components $Y_{1}, \ldots, Y_{k}$ which satisfy

$$
\sum_{i=1}^{k} \operatorname{cat}_{M}\left(Y_{i}\right)<\operatorname{cat}(M,[\omega])
$$

where $[\omega] \in H^{1}(M ; \mathbb{R})$ denotes the cohomology class of $\omega$. Then there exists an integer $N>0$ such that for any gradient-like vector field $v$ for $\omega$ there exists a finite chain of orbits

$$
\gamma_{1}, \ldots, \gamma_{\ell}, \gamma_{\ell+1}=\gamma_{1}
$$

lying in $M-Y$ (with $1 \leq \ell \leq k)$ and labels $1 \leq i(j) \leq k$ such that

$$
\bigcap_{t \in \mathbb{R}} \overline{\gamma_{j}([t,+\infty))} \subset Y_{i(j)} \quad \text { and } \bigcap_{t \in \mathbb{R}} \overline{\gamma_{j+1}((-\infty, t])} \subset Y_{i(j)},
$$

for all $1 \leq j \leq \ell$ and additionally one has

$$
\sum_{i=1}^{\ell} \int_{\gamma_{i}} \omega \leq N .
$$

In other words, if the set of zeros of a closed 1-form $\omega$ is "small" in some sense, i.e. it satisfies the inequality (38), then any gradient-like vector field $v$ for $\omega$ has a generalized homoclinic cycle making at most $N$ full twists with respect to $\omega$.

Note that conditions (39) describe a generalization of the notion of homoclinic cycle. Indeed, (39) intuitively means that the $j$-th trajectory $\gamma_{j}$ "ends" at the same connected component $Y_{i(j)}$ of $Y$ from which the next $(i+1)$-th trajectory $\gamma_{j+1}$ "originates". Such sequence of trajectories $\gamma_{1}, \ldots, \gamma_{\ell}$ will be called a generalized homoclinic cycle.

Theorem 12 reduces to Theorem 8 under an additional assumption that the set $Y$ is finite.

The proof of Theorem 12 is postponed to section 98 , in the following section we prove a theorem which is used in 88 .

\section{EXISTENCE OF FLOW-CONVEX NEIGHBORHOODS}

In this section we study some auxiliary problem which will be used in the proof of Theorems 12 and Theorem 14 and is also of independent interest. The results of this section are typical for the Conley theory of isolated invariant sets. We follow here the paper [17].

\footnotetext{
${ }^{2}$ This condition is automatically satisfied if either the cohomology class $[\omega]$ is integral or $Y$ is an ENR, see Lammas 6, 7
} 


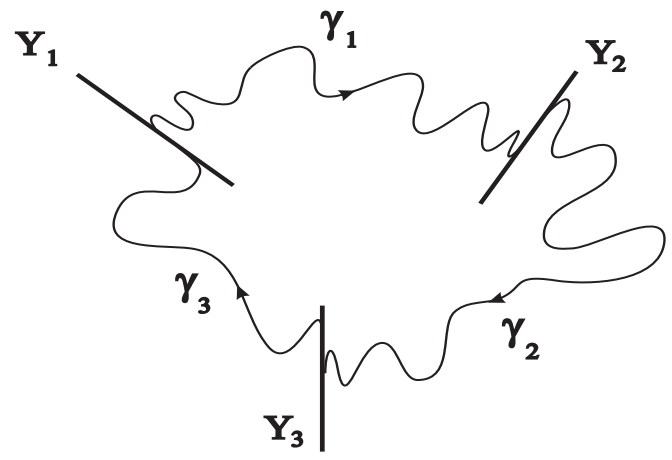

Figure 4. Generalized homoclinic cycle.

Consider a smooth vector field $v$ on a closed smooth manifold $M$. Let $\Phi: M \times \mathbb{R} \rightarrow M$ be the flow of $v$. We will write $\Phi(x, t)$ as $x \cdot t$, where $x \in M$ and $t \in \mathbb{R}$. The symbol $R$ denotes the chain recurrent set of $\Phi$.

Theorem 13 (Farber - Kappeler [17]). Let $Z$ be a connected component of $R$ which is isolated in $R$, i.e. such that there exists a neighborhood $Z \subset U$ with $U \cap R=Z$. Let $W \subset M$ be a neighborhood of $Z$. Then there exists an open neighborhood $B$ of $Z$, contained in $W$, with the following two properties:

(A) For any $x \in M$, the open set

$$
J_{x}=\{t ; x \cdot t \in B\} \subset \mathbb{R}
$$

is convex (i.e. it is either empty or an interval);

(B) Let $A$ be the set of points $x \in M$ such that the interval $J_{x}$ is nonempty and bounded below. Then the function

$$
A \rightarrow \mathbb{R}, \quad x \mapsto \inf J_{x},
$$

is continuous.

A neighborhood $B$ of $Z$ having properties (A) and (B) is called convex with respect to the flow $\Phi$.

Theorem 13 could be compared to Lemma B.1 in Appendix B of [15].

By Conley's theorem [4, 5], there exists a smooth Lyapunov function $L$ : $M \rightarrow \mathbb{R}$ for the flow; see also [20], Proposition 2, where the proof of the smooth version of Conley's theorem is given. The Lyapunov function $L$ satisfies: $V(L)<0$ on the complement $M-R$ of $R$ and the differential $d L$ vanishes on $R$.

Fix a point $x \in M-R$ and consider its orbit $x(t)=x \cdot t$. The function $t \mapsto L(x \cdot t)$ is strictly decreasing. Hence, as $t$ tends to $+\infty$ the limit

$$
\ell(x)=\lim _{t \rightarrow \infty} L(x \cdot t)
$$

exists and is finite. If $x \in R$ then the function $t \mapsto L(x \cdot t)$ is constant. 


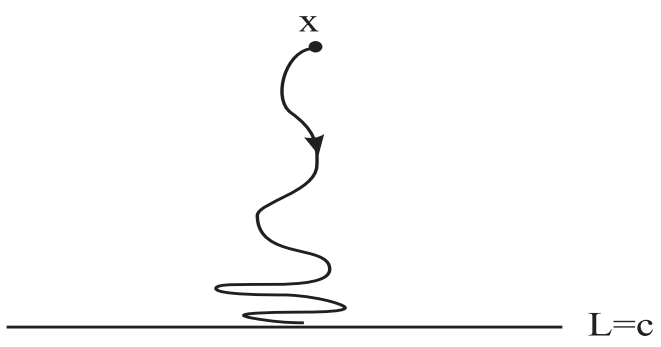

For any $x \in M$ the number $\ell(x)$ is a critical value of $L$. Indeed, the $\omega$-limit set $\omega(x)$ is contained in the level set $L^{-1}(\ell(x))$ and, on the other hand, $\omega(x)$ is a part of the chain recurrent set $R$ which is the set of critical points of $L$.

Lemma 14. The function $\ell: M \rightarrow \mathbb{R}$ is upper semi-continuous: if $x_{n} \rightarrow$ $x \in M$ then

$$
\ell(x) \geq \lim \sup \ell\left(x_{n}\right) .
$$

Proof. Given any $\epsilon>0$ there exists $T>0$ such that $0<L(x \cdot T)-\ell(x)<\epsilon$. Since $L$ is continuous and the map $x \mapsto x \cdot T$ is continuous, there exists a neighborhood $U \subset M$ of $x$ such that $|L(y \cdot T)-L(x \cdot T)|<\epsilon$ for any $y \in U$. Hence for all $y \in U$ one has

$$
\ell(y) \leq L(y \cdot T)<L(x \cdot T)+\epsilon<\ell(x)+2 \epsilon .
$$

Hence, $\ell(y) \leq \ell(x)$.

The function $L: M \rightarrow \mathbb{R}$ restricted to $Z$ is constant. Indeed, $L(Z) \subset \mathbb{R}$ must be connected (as the image of a connected set) and it has measure zero by Sard's theorem. Hence it is a single point. Denote $c=L(Z)$.

Let $Z \subset W$ be an open neighborhood. As $Z$ is supposed to be isolated in $R$, we may assume (without loss of generality) that $\bar{W} \cap R=Z$.

Fix $\epsilon>0$ and denote by $A_{+}(\epsilon)$ the set of all points $x \in M$ with the properties: (a) $L(x)=c+\epsilon$; (b) $\omega(x) \subset Z$. Note that (b) implies that $\ell(x)=c$.

Lemma 15. For any sufficiently small $\epsilon>0$ the set $A_{+}(\epsilon)$ is contained in the neighborhood $W$.

Proof. Assuming the contrary, there exists a convergent sequence $x_{n} \in M-$ $W$ such that $L\left(x_{n}\right) \rightarrow c$ and $L\left(x_{n}\right)>c, \ell\left(x_{n}\right)=c$ and $\omega\left(x_{n}\right) \subset Z$, where $\omega(x)$ denotes the $\omega$-limit set of the trajectory $x \cdot t$. If $x_{0}=\lim x_{n} \in M$ is the limit of $x_{n}$ then (using (42) ) one has $\ell\left(x_{0}\right) \geq c$. On the other hand, $L\left(x_{0}\right)=c$ and hence $\ell\left(x_{0}\right)=c$. We obtain that $x_{0}$ belongs to the set $L^{-1}(c) \cap(R-Z)$. In other words, it lies in a connected component of $R$ distinct from $Z$.

Fix a Riemannian metric on $M$. Denote by $d>0$ the distance between $Z$ and $R-Z$. Denote by $K>0$ a constant such that the norm of the vector $V$ is less than $K$ at every point of $M$. Such $K$ exists since $M$ is compact. 
Fix some $\delta>0$ such that $\delta<d / 2$. The function $V(L): M \rightarrow \mathbb{R}$ restricted to the complement of the $\delta$-neighborhood of $R$ is negative and moreover can be estimated from above $V(L) \leq-\eta$ for some positive $\eta=\eta_{\delta}>0$. Now, we may find a large $n$ such that

$$
L\left(x_{n}\right)-c<\frac{\eta(d-2 \delta)}{K}
$$

and $x_{n}$ lies in the $\delta$-neighborhood of $R-Z$. The trajectory $x_{n} \cdot t$ approaches $Z$ for large $t$. The length of the trajectory is given by

$$
\int_{t_{1}}^{t_{2}}|\dot{x}(t)| d t=\int_{t_{1}}^{t_{2}} \mid V\left(x(t)|d t \leq K| t_{2}-t_{1} \mid\right.
$$

One concludes that the time $\tau_{n}$ the trajectory $x_{n} \cdot t$ for $t>0$ spends in the complement of the $\delta$-neighborhood of $R$ can be estimated by

$$
\tau_{n} \geq \frac{d-2 \delta}{K}
$$

Therefore, for large $t>0$ one has

$$
L\left(x_{n} \cdot t\right)-L\left(x_{n}\right)=\int_{0}^{t} V(L)\left(x_{n} \cdot t\right) d t \leq-\eta \cdot \tau_{n} \leq-\frac{\eta(d-2 \delta)}{K}
$$

These inequalities show (since $L\left(x_{n}\right)$ tends to $c$ ) that for large $t$ one has $L\left(x_{n} \cdot t\right)<c$ contradicting the assumption $\ell\left(x_{n}\right)=c$.

Remark 2. Define $A_{-}(\epsilon)$ as the set of all points $x \in M$ with $L(x)=c-\epsilon$ and $\alpha(x) \subset Z$ where $\alpha(x)$ denotes the $\alpha$-limit set of the trajectory $x \cdot t$. Applying the above arguments to the time reversed flow one obtains that for any neighborhood $W$ of $Z$ the set $A_{-}(\epsilon)$ is contained in $W$ for any sufficiently small $\epsilon>0$.

The arguments of the proof could be summarized as follows: the point $x_{n}$ is very close to a component $Z^{\prime}$ of $R$ lying in $L^{-1}(c)$ and distinct from $Z$. The trajectory starting at $x_{n}$ cannot approach $Z$ since while it passes the distance separating $Z$ and $Z^{\prime}$ it descends with respect to $L$ so that the point $x_{n} \cdot t$ slips below the level $c$.

Lemma 16. For any sufficiently small $\epsilon>0$ the set $A_{+}(\epsilon)$ is closed.

Proof. Let $W$ be a neighborhood of $Z$ with $\bar{W} \cap R=Z$ and let $\epsilon>0$ be such that $A_{+}(\epsilon) \subset W$. Assume that a sequence $x_{n} \in A_{+}(\epsilon)$ converges to a point $x_{0}$. Then, for any $t>0$ the point $x_{n} \cdot t$ lies in $W$. Hence we obtain that for any $t>0$ the point $x_{0} \cdot t$ lies in $\bar{W}$. It follows that $\omega\left(x_{0}\right) \subset \bar{W} \cap R=Z$. Hence, $x_{0}$ is an element of $A_{+}(\epsilon)$.

Lemma 17. Let $W$ be an open neighborhood of $Z$ such that $\bar{W} \cap R=Z$. Let $\epsilon>0$ be such that $A_{+}\left(\epsilon^{\prime}\right)$ is contained in $W$ for any $0<\epsilon^{\prime} \leq \epsilon$. Then there exists an open neighborhood $U_{+}(\epsilon)$ of the set $A_{+}(\epsilon)$ in the level set $L^{-1}(c+\epsilon)$ and an open neighborhood $U_{0}$ of $Z$ in $L^{-1}(c)$ with the following properties: 


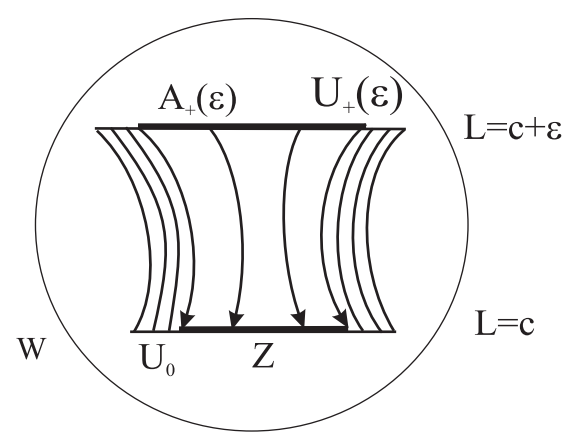

(1) If $x \in U_{+}(\epsilon)-A_{+}(\epsilon)$ then for some $\tau_{x}>0$ the point $x \cdot \tau_{x}$ lies in $U_{0}-Z$.

(2) The mapping $x \mapsto x \cdot \tau_{x}$ is a homeomorphism of $U_{+}(\epsilon)-A_{+}(\epsilon) \rightarrow$ $U_{0}-Z$.

(3) For any $0 \leq t \leq \tau_{x}$ the point $x \cdot t$ lies in $W$.

(4) The number $\tau_{x}$ depends continuously on $x \in U_{+}(\epsilon)-A_{+}(\epsilon)$ and it tends to $+\infty$ as $x \in U_{+}(\epsilon)-A_{+}(\epsilon)$ approaches $A_{+}(\epsilon)$.

Proof. If $L(y)=c+\epsilon$ and $y$ is close enough to the set $A_{+}(\epsilon)$ then for $t>0$ the orbit $y \cdot t$ does not leave $W$ before it leaves the set $L \geq c$. Indeed, if this claim is false then there exists a sequence of points $y_{n} \in M$ and a sequence of numbers $t_{n}>0$ such that $L\left(y_{n}\right)=c+\epsilon, y_{n} \rightarrow x \in A_{+}(\epsilon), L\left(y_{n} \cdot t_{n}\right) \geq c$, and $y_{n} \cdot t_{n} \notin W$. Fix a Riemannian metric on $M$. Let $K>0$ be such that the length of the vector $V(x)$ is less or equal than $K$ at every point $x \in M$. Choose a small neighborhood $G$ of $Z$ such that its closure is contained in $W$. Let $d>0$ be the distance between $\bar{G}$ and $M-W$. The function $V(L)$ satisfies in $W-G$ the inequality $V(L) \leq-\eta$ for some $\eta>0$. Now, let $G^{\prime} \subset G$ be a neighborhood of $Z$ such that

$$
L\left(G^{\prime}\right) \subset\left(-\infty, c+\eta \cdot \frac{d}{K}\right) .
$$

As $x$ belongs to $A_{+}(\epsilon)$ there exists $t^{\prime}>0$ with $x \cdot t^{\prime} \in G^{\prime}$. Then for $n$ large enough $y_{n} \cdot t^{\prime} \in G^{\prime}$. Arguing as in the proof of Lemma 15, one obtains that the point $y_{n} \cdot t$ cannot reach $M-W$ before it leaves the domain $L \geq c$.

Similarly we observe: if $L(y)=c$ where $y \in W-Z$ is sufficiently close to $Z$ then the trajectory $y \cdot t$ for some $t<0$ reaches the level $L=c+\epsilon$ without leaving $W$. Indeed, if this statement is false one finds a sequence $y_{n} \in W-Z, L\left(y_{n}\right)=c$ such that $y_{n} \rightarrow x \in Z$ and for some $t_{n}<0$, $y_{n} \cdot t_{n} \in \partial W, L\left(y_{n} \cdot t_{n}\right) \leq c+\epsilon$. We may assume that the sequence $y_{n} \cdot t_{n}$ converges to a point $z \in \partial W$. If $\ell(z)>c$ the forward trajectory $z \cdot t$ where $t>0$ crosses the level surface $L=c$ at a point which is not in $Z$ and the trajectory $y_{n} \cdot t$ must cross this level at a nearby point as $n \rightarrow \infty$, a contradiction. Hence we have to consider only the case $\ell(z)=c$ which leaves 
two possibilities: either $\omega(z)$ is contained in a connected component of $R$ distinct from $Z$ which also lies on the level surface $L=c$, or $\omega(z) \subset Z$. The first possibility leads to a contradiction, arguing as in the proof of Lemma 15. The second possibility means that $z$ belongs to $A_{+}\left(\epsilon^{\prime}\right)$ where $0<\epsilon^{\prime} \leq \epsilon$. This is a contradiction since we assume that $A_{+}\left(\epsilon^{\prime}\right)$ is contained in the open set $W$.

Let $U_{0}$ be the union of the set $Z$ and the set of all points $y \in W \cap L^{-1}(c)$ such that the trajectory $y \cdot t$ for $t<0$ reaches the set $W \cap L^{-1}(c+\epsilon)$ without leaving $W$.

Let $U_{+}(\epsilon)$ be the union of $A_{+}(\epsilon)$ and the set of all $y \in W \cap L^{-1}(c+\epsilon)$ such that the trajectory $y \cdot t$ for $t>0$ reaches the level surface $L^{-1}(c)$ without leaving $W$.

$U_{0}$ is an open neighborhood of $Z$ in $L^{-1}(c)$ and $U_{+}(\epsilon)$ is an open neighborhood of $A_{+}(\epsilon)$ in $L^{-1}(c+\epsilon)$ as was shown above. It is then easy to see that the statements of Lemma 17 hold for $U_{0}$ and $U_{+}(\epsilon)$. Let us show, for example, that if $x_{n} \in U_{+}(\epsilon)-A_{+}(\epsilon)$ and $x_{n} \rightarrow x \in A_{+}(\epsilon)$ then $\tau_{x_{n}}$ tends to $+\infty$. If not one may pass to a subsequence such that the sequence $\tau_{x_{n}}$ has a finite limit $\tau$. Then $L(x \cdot \tau)=\lim L\left(x_{n} \cdot \tau_{x_{n}}\right)=c$. Thus the trajectory $x \cdot t$ arrives in finite time at $Z$ which is impossible since $Z$ is flow-invariant and $x \notin Z$.

Here is an analog of Lemma 17;

Lemma 18. Let $W$ be an open neighborhood of $Z$ such that $\bar{W} \cap R=Z$. Let $\epsilon>0$ be such that $A_{-}\left(\epsilon^{\prime}\right)$ is contained in $W$ for any $0<\epsilon^{\prime} \leq \epsilon$. Then there exists an open neighborhood $U_{-}(\epsilon)$ of the set $A_{-}(\epsilon)$ in the level set $L^{-1}(c-\epsilon)$ and an open neighborhood $U_{0}$ of $Z$ in $L^{-1}(c)$ with the following properties:

(1) If $x \in U_{-}(\epsilon)-A_{-}(\epsilon)$ then for some $T_{x}<0$ the point $x \cdot T_{x}$ lies in $U_{0}-Z$.

(2) The mapping $x \mapsto x \cdot T_{x}$ is a homeomorphism

$$
U_{-}(\epsilon)-A_{-}(\epsilon) \rightarrow U_{0}-Z \text {. }
$$

(3) For any $T_{x}<t<0$ the point $x \cdot t$ lies in $W$.

(4) The number $T_{x}$ depends continuously on $x \in U_{-}(\epsilon)-A_{-}(\epsilon)$ and it tends to $-\infty$ as $x \in U_{-}(\epsilon)-A_{-}(\epsilon)$ approaches $A_{-}(\epsilon)$.

Proof. It is similar to the proof of Lemma 17.

Proof of Theorem 13. Let $W \subset M$ be an open neighborhood of $Z$. We may assume without loss of generality that $\bar{W} \cap R=Z$. By Lemma 15 and Remark 2 we may find $\epsilon>0$ such that for any $0<\epsilon^{\prime} \leq \epsilon$ the sets $A_{+}\left(\epsilon^{\prime}\right)$ and $A_{-}\left(\epsilon^{\prime}\right)$ are contained in $W$.

Let $C$ denote the set of all points $x \in W \cap L^{-1}(c-\epsilon, c+\epsilon)$ such that there exist numbers $t_{x}<0<\tau_{x}$ with

$$
L\left(x \cdot t_{x}\right)=c+\epsilon, \quad L\left(x \cdot \tau_{x}\right)=c-\epsilon
$$


and $x \cdot t$ is contained in $W$ for any $t \in\left(t_{x}, \tau_{x}\right)$. Observe that if $x \in C$ then $x \cdot\left(t_{x}, \tau_{x}\right)$ is contained in $C$.

Define the set

$$
B=C \cup Z \cup \bigcup_{0<\epsilon^{\prime}<\epsilon} A_{ \pm}\left(\epsilon^{\prime}\right) .
$$

We are going to show that it satisfies the requirements of Theorem 13. This set is open. Indeed, the terms in (43) are pairwise disjoint. The set $C$ is clearly open. Any point of $A_{ \pm}\left(\epsilon^{\prime}\right)$ (where $0<\epsilon^{\prime}<\epsilon$ ) has a neighborhood contained entirely in $B$ (by Lemmas 17 and 18). Similarly, any sufficiently small neighborhood $G$ of a point $x \in Z$ is contained in $B$ : if $y \in G$ and $L(y)>c$ then the trajectory $y \cdot t$ for $t>0$ either approaches $Z$ (in that case $y$ lies in $A_{+}\left(\epsilon^{\prime}\right)$ for some $\left.0<\epsilon^{\prime}<\epsilon\right)$ or it hits the level surface $L=c$. In the second case the intersection point is very close to $Z$ and hence by Lemmas 17 and 18 the trajectory continues all the way till it reaches the level $L=c-\epsilon$ without leaving $W$.

Given $x \in M$ consider the set $J_{x}=\{t ; x \cdot t \in B\}$. Let us assume that $L(x)>c$. Then there exist the following possibilities:

(1) $\ell(x)>c$; then $J_{x}=\emptyset$.

(2) $\left.\omega(x) \subset J_{x}\right)$; then $J_{x}$ is a half infinite interval $(a,+\infty)$.

(3) $\ell(x)=c$ and $\omega(x) \cap Z=\emptyset$; then $J_{x}=\emptyset$.

(4) $\ell(x)<c$; then $J_{x}$ is either the empty set or a finite interval $(a, b)$.

In the case $L(x) \leq c$ the arguments are similar.

The continuity of $x \mapsto \inf J_{x}$ follows from the Implicit Function Theorem: the number $\inf J_{x}=t$ is the solution of the equation $L(x \cdot t)=c+\epsilon$ and the partial derivative of $L(x \cdot t)$ with respect to $t$ is strictly negative.

\section{Proof of Theorem 12}

The arguments are similar to those use in the proof of Theorem 4.1 of [15]. Suppose that Theorem 12 is false, i.e. for any $N>0$ there exists a gradientlike vector field $v=v_{N}$ for $\omega$ having no generalized homoclinic cycles of integral trajectories $\gamma_{1}, \ldots, \gamma_{\ell}$ satisfying (39) and (40). Our goal is to show that then

$$
\operatorname{cat}(M,[\omega]) \leq \sum_{i=1}^{k} \operatorname{cat}_{M}\left(Y_{i}\right),
$$

i.e. the inequality (38) is violated. Without loss of generality we may assume that the number of connected components $k$ is finite since if $k=\infty$ the inequality (44) is obviously true, as $\operatorname{cat}_{M}\left(Y_{i}\right) \geq 1$ for any $i$.

Fix a Riemannian metric on $M$. We will denote the flow $M \times \mathbb{R} \rightarrow M$ generated by $-v$ by $t \mapsto m \cdot t$, where $m \in M$ and $t \in \mathbb{R}$. Since $\omega(v)>0$ on $M-Y$, the integral $\int_{p}^{p \cdot t} \omega \leq 0$ is non-positive and non-increasing for $t>0$. We start by proving the following Lemma: 
Lemma 19. Assume that for an integer $N>0$ there exists a gradientlike vector field $v$ for $\omega$ having no generalized homoclinic cycles of integral trajectories $\gamma_{1}, \ldots, \gamma_{\ell}$ satisfying (39) and (40). Let $Y_{i} \subset U_{i} \subset M$ be an open neighbourhood of $Y_{i}$, where $i=1, \ldots, k$. Then there exists a family of smaller closed neighbourhoods $V_{i} \subset U_{i}$ of $Y_{i}$ satisfying the following properties:

(a) Each set $V_{i}$ lifts to the covering space $\pi: \tilde{M} \rightarrow M$ corresponding to the kernel of the homomorphism of periods $\pi_{1}(M) \rightarrow \mathbb{R}$ determined by the cohomology class $\xi=[\omega] \in H^{1}(M ; \mathbb{R})$.

(b) The lift of $V_{i}$ into $\tilde{M}$ is flow convex (i.e. it satisfies properties (A) and (B) of Theorem 13) with respect to the natural lift $\tilde{v}$ of the flow $v$ into $\tilde{M}$.

(c) Let $\partial_{-} V_{i}$ denote the exit set of $V_{i}$, i.e. the set of all points $p \in V_{i}$ such that for all sufficiently small $\tau>0$ one has $p \cdot \tau \notin V_{i}$. Then there exist no $p \in \partial_{-} V_{i}$ and $t>0$ such that $p \cdot t \in \operatorname{Int} V_{i}$ and $\int_{p}^{p \cdot t} \omega \geq-N$.

The intuitive meaning of property (c) is that it is impossible for a trajectory to leave $V_{i}$ and then revisit $V_{i}$ in time $t>0$ without making the quantity $\int_{p}^{p \cdot t} \omega$ smaller than $-N$.
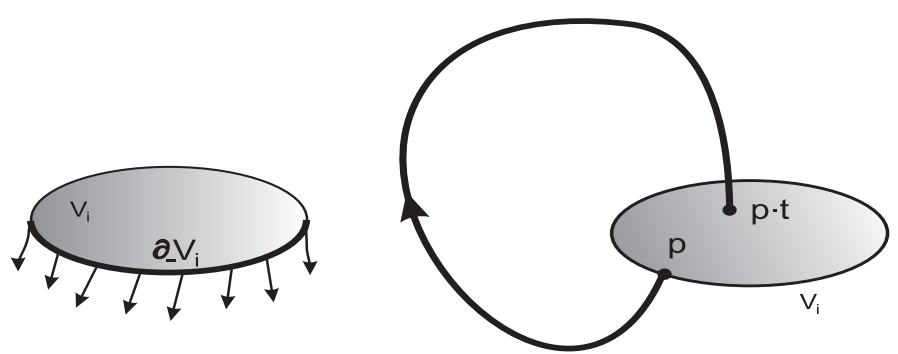

Proof. Without loss of generality we may assume that the initial neighbourhoods $U_{i}$ satisfy properties (a) and (b) of Lemma 19.

Let $V_{i} \subset U_{i}$ be any closed neighborhood of $Y_{i}$ satisfying (b); then (a) is automatically satisfied. Denote by $V$ and $U$ the unions $V=V_{1} \cup \cdots \cup V_{k}$, $U=U_{1} \cup \cdots \cup U_{k}$ and $\partial_{-} V=\partial_{-} V_{1} \cup \cdots \cup \partial_{-} V_{k}$. We claim:

(i) There exists $a>0$ such that for any $p \in \partial_{-} V$ and $t>0$ with $p \cdot t \in V$ one has $t \geq a$.

Indeed, as follows from the gradient convexity of the sets $U_{i}$, any trajectory $p \cdot t$ starting at $p \in \partial_{-} V$ at $t=0$ leaves $U$ before it can re-enter $V$. Hence, we may take $a=\min \left\{l_{i} v_{i}^{-1} ; i=1, \ldots, k\right\}$, where $l_{i}>0$ denotes the distance between $V_{i}$ and $M-\operatorname{Int} U_{i}$, and $v_{i}=\max |v(x)|$ for $x \in U_{i}-\operatorname{Int} V_{i}$.

Note that if we shrink the sets $V_{i}$, the number $a>0$ may only increase, assuming that $V_{i}$ are sufficiently small. 
(ii) There exists $b>0$ such that for any $p \in \partial_{-} V$ and $t>0$ with $p \cdot t \in V$ one has

$$
\int_{p}^{p \cdot t} \omega<-b
$$

Indeed, we may take $b=a \cdot \min \{\omega(v(x)) ; x \in U-V\}$.

From (i) and (ii) it follows that:

(iii) There exists an integer $K>0$ such that for any $p \in \partial_{-} V$ the set

$$
I(p)=\left\{t>0 ; p \cdot t \in V \text { and } \int_{p}^{p \cdot t} \omega \geq-N\right\}
$$

is a union of at most $K$ disjoint intervals, i.e.

$$
I(p)=\bigcup_{s=1}^{k(p)}\left[a_{s}(p), b_{s}(p)\right], \quad k(p) \leq K
$$

and

$$
0<a_{s}(p) \leq b_{s}(p)<a_{s+1}(p) .
$$

Indeed, the number of gaps between the intervals $\left[a_{s}(p), b_{s}(p)\right]$ is at most $[N / b]$ as follows from (ii).

For a point $q \in M$ we denote by

$$
\mathcal{A}(q)=\bigcap_{t \in \mathbb{R}} \overline{q \cdot(-\infty, t]}, \quad \mathcal{Z}(q)=\bigcap_{t \in \mathbb{R}} \overline{q \cdot[t, \infty)},
$$

the backward and forward limit sets of the trajectory passing through $q$. These sets are nonempty, compact, connected and flow-invariant.

Now, suppose that we can never achieve (c) by shrinking the initially chosen neighbourhoods $V_{i} \supset Y_{i}$. We want to show that then there exists a sequence of points $q_{1}, \ldots, q_{\ell} \in M$ and an injection $j:\{1, \ldots, \ell\} \rightarrow\{1, \ldots, k\}$ such that

$$
\mathcal{A}\left(q_{i}\right) \subset Y_{j(i)}, \quad \mathcal{Z}\left(q_{i}\right) \subset Y_{j(i+1)}
$$

where $i=1, \ldots, \ell$ and $j(\ell+1)=j(1)$ and additionally the following inequality is satisfied

$$
\sum_{i=1}^{\ell} \int_{\gamma_{i}} \omega \geq-N
$$

where the curve $\gamma_{i}: \mathbb{R} \rightarrow M$ is given by $\gamma_{i}(t)=q_{i} \cdot t$ for $t \in \mathbb{R}$ which would contradict our assumptions.

There exists an infinite sequence of points $p_{n} \in \partial_{-} V$, where $n=1,2, \ldots$, and two sequences of real numbers $t_{n}>0$ and $s_{n}<0$ such that

(1) the set $p_{n} \cdot\left[s_{n}, 0\right]$ is contained in a fixed connected component $V_{i} \subset V$ and the distance $d\left(p_{n} \cdot s_{n}, Y_{i}\right)$ tends to 0 as $n \rightarrow \infty$;

(2) $d\left(p_{n} \cdot t_{n}, Y_{i}\right)$ converges to 0 as $n \rightarrow \infty$; 
(3) one has

$$
\int_{p_{n}}^{p_{n} \cdot t_{n}} \omega \geq-N .
$$

Passing to a subsequence, we may assume that $p_{n}$ converges to a point $p \in \partial_{-} V_{i}$ and the sequences $s_{n}$ and $t_{n}$ have finite or infinite limits, which we denote by $s$ and $t$ respectively. Because of property (iii), we may also assume that the number of intervals $\kappa\left(p_{n}\right)=\kappa$ is independent of $n$ and the sequences $a_{s}\left(p_{n}\right)$ and $b_{s}\left(p_{n}\right)$ have finite or infinite limits denoted by $a_{s}$ and $b_{s}$ respectively for $s=1, \ldots, \kappa$. Moreover, for the same reasons, we may assume that as $n \rightarrow \infty$ the sequences

$$
p_{n} \cdot a_{s}\left(p_{n}\right) \in V \quad \text { and } \quad p_{n} \cdot b_{s}\left(p_{n}\right) \in V
$$

converge to points $q_{s}, q_{s}^{\prime} \in V$ correspondingly, where $s=1, \ldots, \kappa$.

It is easy to see that (1) and (2) imply that $t=+\infty$ and $s=-\infty$.

Note that $\mathcal{A}(p) \subset Y_{i}$. Indeed, by (1), one has $p \cdot(-\infty, 0] \subset V_{i}$ and therefore the set $\mathcal{A}(p)$ must be contained in the maximal invariant subsets $Y_{i}$ of $V_{i}$. s Clearly the points $q_{s}, q_{s}^{\prime}$ lie on the boundary of the same connected com-

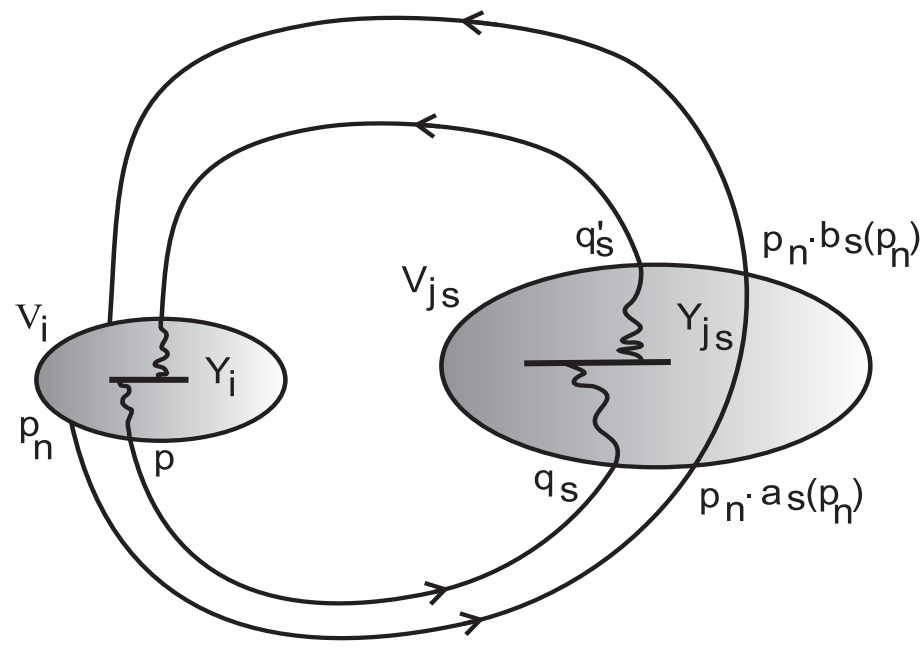

ponent $V_{j_{s}}$ of $V$ where $s \mapsto j_{s}$ is a map $\{1, \ldots, \kappa\} \rightarrow\{1, \ldots, k\}$ and $j_{\kappa}=i$. The points $q_{s}$ and $q_{s}^{\prime}$ either lie on the same orbit of the flow or one has

$$
\mathcal{Z}\left(q_{s}\right) \subset Y_{j_{s}}, \quad \mathcal{A}\left(q_{s}^{\prime}\right) \subset Y_{j_{s}}
$$

as follows from arguments mentioned above. We may ignore the first case since then we may simply relabel the points and apply the following observations. The points $q_{s}^{\prime}$ and $q_{s+1}$ lie on the same orbit and hence we may 
rewrite (48) in the form

$$
\mathcal{Z}\left(q_{s}\right) \subset Y_{j_{s}}, \quad \mathcal{A}\left(q_{s+1}\right) \subset Y_{j_{s}}
$$

where $s=1, \ldots, \kappa-1$. For the last point $q_{\kappa}$ we have

$$
\mathcal{Z}\left(q_{\kappa}\right) \subset Y_{i} \quad \text { and } \quad \mathcal{A}\left(q_{1}\right)=\mathcal{A}(p) \subset Y_{i} .
$$

Hence we obtaine a closed chain of integral trajectories - a generalized homoclinic cycle. Inequality (46) follows from (47) by passing to the limit and observing that the form $\omega \mid V_{j}$ is exact, $\omega \mid V_{j}=d f_{j}$ where $f_{j} \rightarrow \mathbb{R}$ is smooth. Moreover the function $f_{j}$ is constant on $Y_{j}$. Indeed, the image $f_{j}\left(Y_{j}\right) \subset \mathbb{R}$ is connected and has measure zero by Sard's theorem; hence $f_{j}\left(Y_{j}\right)$ is a single point. These remarks imply that

$$
\lim _{n \rightarrow \infty} \int_{p_{n} \cdot a_{s}\left(p_{n}\right)}^{p_{n} \cdot b_{s}\left(p_{n}\right)} \omega=\lim _{T \rightarrow \infty} \int_{q_{s}}^{q_{s} \cdot T} \omega+\lim _{S \rightarrow-\infty} \int_{q_{s}^{\prime} \cdot S}^{q_{s}^{\prime}} \omega
$$

for any $s=1, \ldots, \kappa$.

This completes the proof of Lemma 19.

Proof of Theorem 12. Assume that under the condition of Theorem 12 there exist no generalized homoclinic cycles satisfying (40). Applying Lemma 19 with neighbourhoods $W_{i}$ such that $\operatorname{cat}_{M}\left(W_{i}\right)=\operatorname{cat}\left(Y_{i}\right)$ we obtain a system of neighbourhoods $Y_{i} \subset V_{i}$ satisfying (a)-(c) of Lemma 19 and also $\operatorname{cat}_{M}\left(V_{i}\right)=\operatorname{cat}_{M}\left(Y_{i}\right)$ where $i=1, \ldots, k$.

Define sets $F_{1}, \ldots, F_{k} \subset M$ as follows. We say that $p \in F_{i}$ if for some $t_{p}>0$ the point $p \cdot t_{p}$ belongs to the interior of $V_{i}$, and

$$
\int_{p}^{p \cdot t_{p}} \omega>-N
$$

It is clear that $F_{i}$ is open.

Let us show that $F_{i} \subset M$ can be deformed into $V_{i} \subset F_{i}$. For any point $p \in M$ let $J_{p} \subset \mathbb{R}$ denote the set $J_{p}=\left\{t \geq 0 ; p \cdot t \in V_{i}\right\}$. Because of our assumption about the gradient-convexity of neighborhood $V_{i}$, the set $J_{p}$ is a union of disjoint closed intervals, and some of these intervals may degenerate to a point. Consider the first interval $\left[\alpha_{p}, \beta_{p}\right] \subset J_{p}$. If this interval degenerates to a point (i.e., the trajectory through $p$ touches $V_{j}$ ), then $p$ does not belong to the set $F_{j}$, according to our assumption (c); see above. For the same reason, points of $\partial_{-} V_{j}$ do not belong to $F_{j}$.

Assume now that $p \in F_{j}$ and $p \notin \operatorname{Int} V_{i}$. Then the point $p \cdot t$ lies in the interior of $V_{j}$ for $\alpha_{p}<t<\beta_{p}$. Also, we have

$$
\int_{p}^{p \cdot \alpha_{p}} \omega>-N
$$


The function $\phi_{i}: F_{i} \rightarrow \mathbb{R}$, given by

$$
\phi_{i}(p)=\left\{\begin{array}{cl}
0, & \text { for } \quad p \in \operatorname{Int} V_{i} \\
\alpha_{p}, & \text { for } \quad p \in F_{i}-\operatorname{Int} V_{i}
\end{array}\right.
$$

is continuous since $\left(\partial_{-} V_{i}\right) \cap F_{i}=\emptyset$. One may now define a homotopy

$$
h_{\tau}^{i}: F_{i} \rightarrow M, \quad h_{\tau}(p)=p \cdot\left(\tau \phi_{i}(p)\right), \quad p \in F_{i}, \quad \tau \in[0,1] .
$$

Here $h_{0}$ is the inclusion $F_{i} \rightarrow M$ and $h_{1}$ maps $F_{i}$ into the interior of $V_{i}$. By Lemma 19.5 from [7] we obtain

$$
\operatorname{cat}_{M}\left(F_{i}\right) \leq \operatorname{cat}_{M}\left(V_{i}\right)=\operatorname{cat}_{M}\left(Y_{i}\right)
$$

and therefore

$$
\operatorname{cat}_{M}\left(\bigcup_{i=1}^{k} F_{i}\right) \leq \sum_{i=1}^{k} \operatorname{cat}_{M}\left(Y_{i}\right)
$$

Consider now the complement $A=M-\bigcup_{i=1}^{k} F_{i}$. For any point $p \in A$ there exists $t_{p}>0$ such that $\int_{p}^{p \cdot t_{p}} \omega=-N$. It is clear that $p \mapsto t_{p}$ is a continuous real valued function on $A$. One may define a homotopy

$$
h_{\tau}: A \rightarrow A \quad \text { by } \quad h_{\tau}(p)=p \cdot\left(\tau t_{p}\right), \quad \tau \in[0,1] .
$$

Then for any $x \in A$ one has

$$
\int_{x}^{h_{1}(x)} \omega=-N
$$

i.e. the closed set $A$ is $N$-movable with respect to $\omega$ and

$$
\operatorname{cat}_{M}(M-A) \leq \sum_{i=1}^{k} \operatorname{cat}_{M}\left(Y_{i}\right)
$$

According to Definition 7 we obtain that

$$
\operatorname{cat}(M,[\omega]) \leq \sum_{i=1}^{k} \operatorname{cat}_{M}\left(Y_{i}\right)
$$

as claimed. This completes the proof.

\section{Topology of the Chain ReCurrent Set $R_{\xi}$}

In this section we prove the following result from [17].

Theorem 14 (Farber - Kappeler). Consider a smooth flow $\Phi: M \times \mathbb{R} \rightarrow M$ on a closed smooth manifold $M$. Let $\xi \in H^{1}(M ; \mathbb{R})$ be a cohomology class such that the following conditions are satisfied:

(1) The chain recurrent set $R_{\xi}$ is isolated in the full chain recurrent set $R$ of $\Phi$. 
(2) The restriction

$$
\left.\xi\right|_{R_{\xi}} \in \check{H}^{1}\left(R_{\xi} ; \mathbb{R}\right)
$$

(viewed as a Čech cohomology class) vanishes.

(3) For any $\Phi$-invariant, positive Borel measure $\mu$ on $M$ with $\mu(R)>\mu\left(R_{\xi}\right)$, Schwartzman's asymptotic cycle $\mathcal{A}_{\mu}=\mathcal{A}_{\mu}(\Phi) \in H_{1}(M ; \mathbb{R})$ satisfies

$$
\left\langle\xi, \mathcal{A}_{\mu}\right\rangle<0 \text {. }
$$

Then one has the following inequality

$$
\sum_{i=1}^{r} \operatorname{cat}_{M}\left(R_{\xi}^{i}\right) \geq \operatorname{cat}_{s}^{1}(M, \xi),
$$

where $R_{\xi}^{1}, \ldots, R_{\xi}^{r}$ denote the connected component 3 of the chain recurrent set $R_{\xi}$.

A proof of Theorem 14 is given in section 10.

Let us compare Theorem 14 with Theorem 6.6 of [16]. Theorem 14 allows arbitrary sets $R_{\xi}$ whereas Theorem 6.6 of [16] assumes that $R_{\xi}$ consists of finitely many isolated points. Another important advantage of Theorem 14 is that it does not require condition $(*)$, see [16], page 108; this condition is difficult to check in concrete examples. On the other hand, since $\operatorname{cat}_{s}^{1}(X, \xi) \leq \operatorname{Cat}(X, \xi)$, the estimate of Theorem 6.6 of [16] is potentially slightly sharper.

Next we state some corollaries of Theorem 14,

In the special case $\xi=0$ one has $R_{\xi}=R$ and the assumptions (1), (2), (3) of Theorem 14 are automatically satisfied. Hence we obtain the following result which presumably is well known but for which we failed to find a reference:

Theorem 15. Consider a smooth flow on a closed smooth manifold $M$. Let $R$ be the chain recurrent set of the flow. Then

$$
\sum_{i=1}^{r} \operatorname{cat}_{M}\left(R^{i}\right) \geq \operatorname{cat}(M) .
$$

Here $R^{1}, \ldots, R^{r}$ denote the connected components of $R$.

In the special case where the chain recurrent set $R$ consists of finitely many points (the fixed points of the flow) Theorem 15] says that the number of fixed points is at least the Lusternik - Schnirelman category of $M$. This is one of the fundamental results of the classical Lusternik - Schnirelman theory, see [7.

\footnotetext{
${ }^{3}$ The number of connected components $r$ of $R_{\xi}$ can be infinite. In this case inequality (14) is trivially satisfied as its LHS is infinite.
} 
Consider another special case of Theorem 14 when $R_{\xi}$ consists of finitely many points (they are the fixed points of the flow). Condition (2) of Theorem 14 is automatically satisfied under these assumptions. We obtain:

Theorem 16 (Farber - Kappeler). Consider a smooth flow on a closed smooth manifold $M$. Let $\xi \in H^{1}(M ; \mathbb{R})$ be a cohomology class such that the chain recurrent set $R_{\xi}$ consists of finitely many points which are isolated in the full chain recurrent set $R$ of the flow. Assume additionally that condition (3) of Theorem 14 is satisfied. Then the flow has at least $\operatorname{cat}_{s}^{1}(M, \xi)$ fixed points.

Here is a reformulation of Theorem 16 :

Theorem 17 (Farber - Kappeler). Let $\xi \in H^{1}(M ; \mathbb{R})$ be a cohomology class. Consider a flow $\Phi$ on $M$ such that the chain recurrent set $R_{\xi}$ consists of less than cat $_{s}^{1}(M, \xi)$ fixed points and such that condition (3) of Theorem 14 is satisfied. Then at least one of the fixed points of the flow is not isolated in the full chain recurrent set $R$.

Here is a typical application of Theorem 16:

Theorem 18 (Farber - Kappeler). Let $\Phi$ be a smooth flow on a closed smooth manifold such that the chain recurrent set $R$ of $\Phi$ is a union of finitely many circles and isolated points. Let $\xi \in H^{1}(M ; \mathbb{R})$ be a cohomology class such that $\langle\xi, z\rangle \leq 0$ for the homology class $z \in H_{1}(M)$ of any periodic orbit. Then

$$
p_{0}+p_{1}+2 p_{2} \geq \operatorname{cat}_{s}^{1}(M, \xi),
$$

where $p_{0}$ denotes the number of fixed points of the flow, $p_{1}$ denotes the number of periodic orbits which are null-homotopic in $M$, and $p_{2}$ denotes the number of periodic orbits which are homotopically nontrivial but their homology classes $z \in H_{1}(M)$ satisfy $\langle\xi, z\rangle=0$.

Proof. In the special case of Theorem 18 condition (3) of Theorem 14 is equivalent to the requirement that $\langle\xi, z\rangle \leq 0$ for the homology class $z \in$ $H_{1}(M)$ of any periodic orbit. Under the assumptions of Theorem 18 the set $R_{\xi}$ is the union of the fixed points and of the periodic orbits satisfying $\langle\xi, z\rangle=0$. If $C$ is a periodic orbit then the number cat ${ }_{M}(C)$ equals 1 or 2 depending on whether $C$ is or is not null-homotopic in $M$. These consideration show that Theorem 18 follows from Theorem 14 .

As a side remark we point out that under the conditions of Theorem 18 ,

$$
p_{0}+p_{1}+2 p_{2}+2 q_{2} \geq \operatorname{cat}(M)
$$

as follows from Theorem 15, here $q_{2}$ denotes the number of periodic orbits satisfying $\langle\xi, z\rangle \neq 0$. 


\section{Proof of Theorem 14}

Consider a smooth flow $\Phi: M \times \mathbb{R} \rightarrow M, \Phi(x, t)=x \cdot t$ and a real cohomology class $\xi \in H^{1}(M ; \mathbb{R})$ satisfying the conditions of Theorem 14 .

If the chain recurrent set $R_{\xi}$ has infinitely many connected components then the RHS of inequality (51) is infinite and so this inequality is obviously satisfied. Hence without loss of generality we may assume that the number of connected components of $R_{\xi}$ is finite.

Let $R_{\xi}=R_{\xi}^{1} \cup R_{\xi}^{2} \cup \cdots \cup R_{\xi}^{r}$ be the connected components of $R_{\xi}$. Fix mutually disjoint open neighborhoods $W_{i} \supset R_{\xi}^{i}$, where $i=1,2, \ldots, r$. We shall assume that $W_{i}$ is so small that

$$
\operatorname{cat}_{M}\left(W_{i}\right)=\operatorname{cat}_{M}\left(R_{\xi}^{i}\right), \quad i=1, \ldots, r .
$$

Compare [7], Lemmas 19.2 and 19.6.

Applying Theorem 13 we may find an open neighborhood $B_{i} \subset W_{i}$ of $R_{\xi}^{i}$ which is convex with respect to the flow $\Phi$. Clearly,

$$
\operatorname{cat}_{M}\left(\bar{B}_{i}\right)=\operatorname{cat}_{M}\left(R_{\xi}^{i}\right), \quad i=1, \ldots, r,
$$

as a consequence of (55).

Let $U_{i}$ be an open neighborhood of $R_{\xi}^{i}$ such that the closure $\bar{U}_{i}$ is contained in $B_{i}$. We have the inclusions $R_{\xi}^{i} \subset U_{i} \subset \bar{U}_{i} \subset B_{i} \subset W_{i}$.

Let $A \subset M$ denote the set of points $x \in M$ such that for any $t \in \mathbb{R}$ the point $x \cdot t$ does not belong to the union $U=\cup_{j=1}^{r} U_{j}$. The set $A$ is closed and flow-invariant.

Applying Theorem [5] we find that there exists a smooth Lyapunov 1-form $\omega$ for the pair $\left(\Phi, R_{\xi}\right)$ lying in the cohomology class $\xi=[\omega] \in H^{1}(M ; \mathbb{R})$. This means that the function $\omega(V): M \rightarrow \mathbb{R}$ is negative on $M-R_{\xi}$ (where $V$ denotes the vector field on $M$ generating the flow $\Phi)$ and $\left.\omega(V)\right|_{R_{\xi}}=0$. Moreover, the restriction of $\omega$ on some open neighborhood of $R_{\xi}$ is the differential of a smooth function (this is equivalent to condition (1) of Theorem 14).

Let us show that for any integer $N$ the set $A$ is $N$-movable with respect to both $\omega$ and $-\omega$. Since $M-U$ is compact there exists $\epsilon>0$ such that $\omega(V)<-\epsilon$ on $M-U$. Then for any $x \in A$ one has

$$
\int_{x}^{x \cdot t} \omega<-\epsilon t, \text { for } t>0 .
$$

Hence a continuous homotopy $h_{t}: A \rightarrow M, t \in[0,1]$ defined by

$$
h_{t}(x)=x \cdot\left(\frac{N t}{\epsilon}\right)
$$


satisfies the condition of Definition 6 . This shows that $A$ is $N$-movable with respect to $\omega$. In a similar way one shows that for any integer $N$ the set $A$ is $N$-movable with respect to $-\omega$.

Now the inequality (51) follows once one shows that

$$
\operatorname{cat}_{M}(M-A) \leq \sum_{j=1}^{r} \operatorname{cat}_{M}\left(R_{\xi}^{j}\right)
$$

see Definition 8 ,

Let $F_{i} \subset M$ denote the set of points $x \in M$ such that $x \cdot t$ belongs to $B_{i}$ for some $t \in \mathbb{R}$. Then $J_{x}^{i}=\left\{t \in \mathbb{R} ; x \cdot t \in B_{i}\right\}$ is a nonempty open interval. For $x \in F_{i}$ define

$$
\tau_{i}(x)=\left\{\begin{array}{lll}
0, & \text { if } \quad 0 \in J_{x}^{i}, \\
\inf J_{x}^{i}, & \text { if } \quad J_{x}^{i} \subset(0, \infty), \\
\sup J_{x}^{i}, & \text { if } \quad J_{x}^{i} \subset(-\infty, 0) .
\end{array}\right.
$$

The function $\tau_{i}: F_{i} \rightarrow \mathbb{R}$ is continuous as follows from Theorem 13 .

The mapping $x \mapsto x \cdot \tau_{i}(x)$ is a deformation retraction $F_{i} \rightarrow \bar{B}_{i}$ and hence

$$
\operatorname{cat}_{M}\left(F_{i}\right) \leq \operatorname{cat}_{M}\left(\bar{B}_{i}\right)=\operatorname{cat}_{M}\left(R_{\xi}^{i}\right)
$$

in view of (56). Here we use Lemma 19.5 from [7. Since $M-A$ is contained in the union $\cup_{i=1}^{r} F_{i}$ one obtains

$$
\operatorname{cat}_{M}(M-A) \leq \sum_{i=1}^{r} \operatorname{cat}_{M}\left(F_{i}\right) \leq \sum_{i=1}^{r} \operatorname{cat}_{M}\left(R_{\xi}^{i}\right) .
$$

This proves inequality (57) and hence completes the proof of Theorem 14

\section{Cohomological estimates for $\operatorname{cat}(X, \xi)$}

The effectiveness of dynamical applications described in sections $\S ₫ 6$ - 10 depend crucially on the ability to calculate, or to estimate from below, the quantity $\operatorname{cat}(X, \xi)$ where $X$ is a finite polyhedron and $\xi \in H^{1}(X ; \mathbb{R})$ is a cohomology class. The situation here is quite similar to the classical Lusternik - Schnirelmann theory where cat $(X)$ is most often computed by a combination of upper and lower bounds and the most popular lower bound for cat $(X)$ uses the cohomological cup-length, see [7].

In this section we discuss results of this type for $\operatorname{cat}(X, \xi)$ following our paper 24]; some lower bounds for $\operatorname{cat}(X, \xi)$ were obtained earlier in the papers [15] and 18 . We give in this section the main definitions, state principal results and illustrate them by several specific examples; however for complete proofs we refer the reader to our original papers [24], [25] and [23].

Let $X$ be a finite polyhedron and $\xi \in H^{1}(X ; \mathbb{R})$. Denote by $\operatorname{Ker}(\xi)$ the kernel of the homomorphism $\pi_{1}(X) \rightarrow \mathbb{R}$ given by evaluation on $\xi$. Then $H=\pi_{1}(X) / \operatorname{Ker}(\xi)$ is a free abelian group of finite rank $r$ where $r$ denotes the rank of class $\xi$. Consider the cover $p: \tilde{X} \rightarrow X$ corresponding to $\operatorname{Ker}(\xi)$. It has $H$ as the group of covering translations. 
Let $\mathcal{V}_{\xi}=\left(\mathbb{C}^{*}\right)^{r}=\operatorname{Hom}\left(H, \mathbb{C}^{*}\right)$ denote the variety of all complex flat line bundles $L$ over $X$ such that the induced flat line bundle $p^{*} L$ on $\tilde{X}$ is trivial. If $t_{1}, \ldots, t_{r} \in H$ is a basis, then the monodromy of $L \in \mathcal{V}_{\xi}$ along $t_{i}$ is a nonzero complex number $x_{i} \in \mathbb{C}^{*}$ and the numbers $x_{1}, \ldots, x_{r} \in \mathbb{C}^{*}$ form a coordinate system on $\mathcal{V}_{\xi}$. Given a flat line bundle $L \in \mathcal{V}_{\xi}$ the monodromy representation of $L$ is the ring homomorphism

$$
\operatorname{Mon}_{L}: \mathbb{C}[H] \rightarrow \mathbb{C}
$$

sending each $t_{i} \in H$ to $x_{i} \in \mathbb{C}^{*}$. The dual bundle $L^{*} \in \mathcal{V}_{\xi}$ is such that $L \otimes L^{*}$ is trivial; if $x_{1}, \ldots, x_{r} \in \mathbb{C}^{*}$ are coordinates of $L$ then $x_{1}^{-1}, \ldots, x_{r}^{-1} \in \mathbb{C}^{*}$ are coordinates of $L^{*}$.

Any nontrivial element $p \in \mathbb{C}[H]$ lying in the kernel of $\operatorname{Mon}_{L}$ can be viewed as a (Laurent) polynomial equation between the variables $x_{1}, \ldots, x_{r}$. Alternatively, we will consider algebraic subvarieties $V \subset \mathcal{V}_{\xi}$. Any such $V$ is the set of all solutions of a system of equations of the form

$$
q_{i}\left(x_{1}, \ldots, x_{r}, x_{1}^{-1}, \ldots, x_{r}^{-1}\right)=0, \quad i=1, \ldots, m
$$

where $q_{i}$ is a Laurent polynomial with complex coefficients

$$
p_{i} \in \mathbb{C}\left[x_{1}, \ldots, x_{r}, x_{1}^{-1}, \ldots, x_{r}^{-1}\right] .
$$

This is equivalent to fixing an ideal $J \subset \mathbb{Q}[H]$ and studying the set of all flat line bundles $L \in \mathcal{V}_{\xi}$ such that $\operatorname{Mon}_{L}(J)=0$.

Definition 12. (1) We say that a bundle $L \in \mathcal{V}_{\xi}$ is $\xi$-algebraic if the monodromy homomorphism $\operatorname{Mon}_{L}: \mathbb{Z}[H] \rightarrow \mathbb{C}$ has nontrivial kernel.

(2) We say that $L \in \mathcal{V}_{\xi}$ is a $\xi$-algebraic integer if the kernel of $\operatorname{Mon}_{L}$ : $\mathbb{Z}[H] \rightarrow \mathbb{C}$ contains a nontrivial polynomial $P \in \mathbb{Z}[H]$ with $\xi$-top coefficient 1 (see below).

(3) We say that $L$ is $\xi$-transcendental if $\operatorname{Mon}_{L}: \mathbb{Z}[H] \rightarrow \mathbb{C}$ is injective.

Any nonzero $P \in \mathbb{Z}[H]$ can be written as $P=\sum_{i=1}^{k} \alpha_{i} h_{i}$ where $\alpha_{i} \in \mathbb{Z}$, $\alpha_{i} \neq 0, h_{i} \in H$ and $\xi\left(h_{1}\right)<\xi\left(h_{2}\right)<\cdots<\xi\left(h_{k}\right)$. The nonzero integer $\alpha_{k} \in \mathbb{Z}$ is called the $\xi$-top coefficients of $P$.

If $t_{1}, \ldots, t_{r} \in H$ is a basis and if $a_{i} \in \mathbb{C}$ denotes the monodromy of $L$ along $t_{i}$, i.e. $a_{i}=\operatorname{Mon}_{L}\left(t_{i}\right)$, then $L$ is $\xi$-algebraic iff there exist a nontrivial Laurent polynomial equation with integral coefficients $q\left(t_{1}, \ldots, t_{r}\right)$ such that $q\left(a_{1}, \ldots, a_{r}\right)=0$.

There exist countably many nonzero Laurent polynomials $q$ with integral coefficients and for each such $q$ the set of solutions $q\left(a_{1}, \ldots, a_{r}\right)=0$ is nowhere dense in $\mathcal{V}_{\xi}$. Since $\mathcal{V}_{\xi}=\left(\mathbb{C}^{*}\right)^{r}$ is homeomorphic to a complete metric space we obtain:

Lemma 20. The set of all $\xi$-transcendental bundles $L \in \mathcal{V}_{\xi}$ is of Baire category 2. In particular, the set of $\xi$-transcendental $L \in \mathcal{V}_{\xi}$ is dense in the variety $\mathcal{V}_{\xi}$. 
Lemma 21. For any $\xi$-transcendental flat line bundle $L \in \mathcal{V}_{\xi}$ the dimension of the vector space $H^{q}(X ; L)$ equals the q-dimensional Novikov - Betti number $b_{q}(\xi)$,

$$
\operatorname{dim} H^{q}(X ; L)=b_{q}(\xi)
$$

Proof. The monodromy homomorphism $\operatorname{Mon}_{L}: \mathbb{Z}[H] \rightarrow \mathbb{C}$ defines a left $\mathbb{Z}[H]$-module structure $\mathbb{C}_{L}$ on $\mathbb{C}$ and by definition

$$
H^{q}(X ; L)=H^{q}\left(\operatorname{Hom}_{\mathbb{Z}[H]}\left(C_{*}(\tilde{X}), \mathbb{C}_{L}\right)\right)
$$

where $C_{*}(\tilde{X})$ is the cellular chain complex of the covering $\tilde{X} \rightarrow X$ corresponding to $\operatorname{Ker}(\xi)$. If $L$ is transcendental then $\operatorname{Mon}_{L}$ gives a field extension $Q(H) \rightarrow \mathbb{C}$ where $Q(H)$ is the field of fractions of $\mathbb{Z}[H]$. We obtain therefore (using finiteness of $C_{*}(\tilde{X})$ over $\mathbb{Z}[H]$ and (59)):

$$
\begin{aligned}
H^{q}(X ; L) & \simeq H^{q}\left(\operatorname{Hom}_{\mathbb{Z}[H]}\left(C_{*}(\tilde{X}) ; Q(H)\right)\right) \otimes_{Q(H)} \mathbb{C}_{L} \\
& \simeq H^{q}(X ; Q(H)) \otimes_{Q(H)} \mathbb{C}_{L} .
\end{aligned}
$$

This implies that for any transcendental $L$ one has

$$
\operatorname{dim}_{\mathbb{C}} H^{q}(X ; L)=\operatorname{dim}_{Q(H)} H^{q}(X ; Q(H))
$$

and the right hand side. Finally we invoke Proposition 1.30 of [18] which explains why the right hand side of (60) equals the Novikov - Betti number $b_{q}(\xi)$.

Theorem 19 (Farber - Schütz, 24]). Let $X$ be a finite cell complex and $\xi \in H^{1}(X ; \mathbb{R})$. Let $L \in \mathcal{V}_{\xi}$ be $\xi$-transcendental. Assume that there exist cohomology classes $v_{0} \in H^{d_{0}}(X ; L)$ and $v_{i} \in H^{d_{i}}(X ; \mathbb{C})$ where $i=1, \ldots, k$ such that $d_{i}>0$ for $i \in\{1, \ldots, k\}$ and the cup-product

$$
v_{0} \cup v_{1} \cup \cdots \cup v_{k} \neq 0 \in H^{*}(X ; L)
$$

is nontrivial. Then $\operatorname{cat}(X, \xi)>k$.

Theorem 19 combines simplicity with remarkable efficiency. Below in this section we test this theorem in many specific examples.

11.1. The notion of cup-length $\operatorname{cl}(X, \xi)$. In view of Theorem 19 we introduce the following notation.

Let $X$ be a finite cell complex and $\xi \in H^{1}(X ; \mathbb{R})$. We denote by $\operatorname{cl}(X, \xi)$ the maximal integer $k \geq 0$ such that Theorem [19] could be applied to $(X, \xi)$; if Theorem 19 is not applicable (i.e. if $H^{*}(X ; L)=0$ for any $\xi$-transcendental $L \in \mathcal{V}_{\xi}$, compare Lemma 21) we set

$$
\operatorname{cl}(X, \xi)=-1 .
$$

Hence,

$$
\operatorname{cl}(X, \xi) \in\{-1,0,1, \ldots\} .
$$

In other words, $\operatorname{cl}(X, \xi) \geq k$ where $k \geq 0$ iff there exists a $\xi$-transcendental flat line bundle $L \in \mathcal{V}_{\xi}$ and there exist cohomology classes $v_{0} \in H^{d_{0}}(X ; L)$ 
and $v_{i} \in H^{d_{i}}(X ; \mathbb{C})$ where $i=1, \ldots, k$ and $d_{i}>0$ for $i \in\{1, \ldots, k\}$ such that the cup-product

$$
v_{0} \cup v_{1} \cup \cdots \cup v_{k} \neq 0 \in H^{*}(X ; L)
$$

is nontrivial.

Note that for $\xi=0$ the number $\operatorname{cl}(X, \xi)$ coincides with the usual cup-length $\operatorname{cl}(X)$; recall that the later is defined as the largest integer $r$ such that there exist cohomology classes $u_{i} \in H^{d_{i}}(X ; \mathbb{C})$ where $i=1, \ldots, r$ of positive degree such that their cup-product $u_{1} \cup \cdots \cup u_{k} \neq 0 \in H^{*}(X ; L)$ is nontrivial.

One can restate Theorem 19 as follows:

Theorem 20. For any finite complex $X$ and $\xi \in H^{1}(X ; \mathbb{R})$ one has

$$
\operatorname{cat}(X, \xi) \geq \operatorname{cl}(X, \xi)+1 \text {. }
$$

The next useful Lemma suggests several different ways to characterize the number $\operatorname{cl}(X, \xi)$. This Lemma plays an important role in the sequel.

Lemma 22. Let $X$ be a finite cell complex and $\xi \in H^{1}(X ; \mathbb{R})$. The following statements regarding an integer $k \geq 0$ are equivalent:

(A) $\operatorname{cl}(X, \xi) \geq k$;

(B) There exists cohomology class $v_{0} \in H^{d_{0}}(X ; L)$ where $L \in \mathcal{V}_{\xi}$ is a $\xi$-transcendental flat line bundle and there exist $k$ integral cohomology classes $v_{i} \in H^{d_{i}}(X ; \mathbb{Z})$ where $i=1, \ldots, k$ and $d_{i}>0$ for $i \in\{1, \ldots, k\}$, such that the cup-product (63) is nontrivial.

(C) Let $H$ denote $H_{1}(X ; \mathbb{Z}) / \operatorname{Ker}(\xi)$ and let $Q(H)$ denote the field of fractions of the group ring $\mathbb{Z}[H]$. Then there exist cohomology classes $w_{0} \in H^{d_{0}}(X ; Q(H))$ and $v_{i} \in H^{d_{i}}(X ; \mathbb{Z})$ where $d_{i}>0$ for $i=$ $1, \ldots, k$ such that the cup-product

$$
w_{0} \cup v_{1} \cup \cdots \cup v_{k} \neq 0 \in H^{*}(X ; Q(H))
$$

is nontrivial.

(D) For any $\xi$-transcendental flat line bundle $L \in \mathcal{V}_{\xi}$ there exists cohomology class $v_{0} \in H^{d_{0}}(X ; L)$ such that the cup-product (63) is nontrivial for some integral cohomology classes $v_{i} \in H^{d_{i}}(X ; \mathbb{Z})$ with $d_{i}>0$ for $i \in\{1, \ldots, k\}$.

(E) For any $\xi$-transcendental flat line bundle $L \in \mathcal{V}_{\xi}$ there exist cohomology classes $v_{0} \in H^{d_{0}}(X ; L)$ and $v_{i} \in H^{d_{i}}(X ; \mathbb{C})$ where $i=1, \ldots, k$ and $d_{i}>0$ for $i \in\{1, \ldots, k\}$ such that the cup-product (63) is nontrivial.

Proof. Let us show that $(\mathrm{A}) \Longrightarrow(\mathrm{B})$. Fix a $\xi$-transcendental bundle $L \in \mathcal{V}_{\xi}$, and $v_{0} \in H^{*}(X ; L)$ such that (63) is nontrivial for some $v_{i} \in H^{d_{i}}(X ; \mathbb{C})$ with $d_{i}>0$. Consider now the cup-products

$$
v_{0} \cup v_{1}^{\prime} \cup \cdots \cup v_{k}^{\prime}
$$


with arbitrary integral cohomology classes $v_{i}^{\prime} \in H^{d_{i}}(X ; \mathbb{Z})$; here the degrees $d_{i}$ are assumed to be fixed. (64) is a multi-linear function of the classes $v_{i}^{\prime}$. Since the integral classes generate $H^{d_{i}}(X ; \mathbb{C})$ over $\mathbb{C}$ we obtain that (64) must be nonzero for some choice of classes $v_{i}^{\prime}$, i.e. (B) holds.

Now we show that $(\mathrm{B}) \Longrightarrow(\mathrm{C})$. Fix $L \in \mathcal{V}_{\xi}$ and the classes $v_{0} \in H^{d_{0}}(X ; L)$ and $v_{i} \in H^{d_{i}}(X ; \mathbb{Z})$ satisfying conditions described in (B). The monodromy homomorphism $\operatorname{Mon}_{L}: \mathbb{Z}[H] \rightarrow \mathbb{C}$ is an injective ring homomorphism, it extends to the field of fractions $Q(H) \rightarrow \mathbb{C}$. The image of the induced homomorphism on cohomology

$$
\psi: H^{d_{0}}(X ; Q(H)) \rightarrow H^{d_{0}}(X ; L)
$$

generates $H^{d_{0}}(X ; L)$ over $\mathbb{C}$ and (65) is injective (for reasons mentioned in the proof of Lemma 21). Fix cohomology classes $v_{i} \in H^{*}(X ; \mathbb{Z})$ where $i=1, \ldots, k$. For a cohomology class $w_{0} \in H^{d_{0}}(X ; Q(H))$ the function

$$
\psi\left(w_{0}\right) \cup v_{1} \cup \cdots \cup v_{k}=\psi\left(w_{0} \cup v_{1} \cup \cdots \cup v_{k}\right) \in H^{*}(X ; L)
$$

extends to a $\mathbb{C}$-linear function of $v_{0} \in H^{d_{0}}(X ; L)$

$$
v_{0} \mapsto v_{0} \cup v_{1} \cup \cdots \cup v_{k} \in H^{*}(X ; L) .
$$

If this function is nonzero then it may not vanish on the image of $\psi$, i.e. (C) holds.

The implication $(\mathrm{C}) \Longrightarrow(\mathrm{D})$ follows from injectivity of homomorphism (65) and from (66).

Implications $(\mathrm{D}) \Longrightarrow(\mathrm{E})$ and $(\mathrm{E}) \Longrightarrow(\mathrm{A})$ are obvious. This completes the proof.

Lemma 23. Assume that $X$ and $Y$ are path connected finite cell complexes and $\xi \in H^{1}(X \times Y ; \mathbb{R})$. Then

$$
\operatorname{cl}(X \times Y, \xi) \geq \operatorname{cl}\left(X,\left.\xi\right|_{X}\right)+\operatorname{cl}\left(Y,\left.\xi\right|_{Y}\right) .
$$

Proof. Denote $\operatorname{cl}\left(X,\left.\xi\right|_{X}\right)=k$ and $\operatorname{cl}\left(Y,\left.\xi\right|_{Y}\right)=r$. Any flat line bundle $L$ over $X \times Y$ has the form $L_{1} \otimes L_{2}$ (exterior tensor product) where $L_{1}$ and $L_{2}$ are flat line bundles over $X$ and $Y$ respectively. Note that if $L$ lies in the variety $\mathcal{V}_{\xi}=\operatorname{Hom}\left(H_{1}(X \times Y ; \mathbb{Z}) / \operatorname{Ker}(\xi), \mathbb{C}^{*}\right)$ then $L_{1}$ and $L_{2}$ are obtained by restrictions and hence $L_{1} \in \mathcal{V}_{\left.\xi\right|_{X}}$ and $L_{2} \in \mathcal{V}_{\left.\xi\right|_{Y}}$. We will use equivalence between (A) and (E) of Lemma 22, Fix a $\xi$-transcendental bundle $L=L_{1} \otimes L_{2} \in \mathcal{V}_{\xi}$ over $X \times Y$. Then both $L_{1}$ and $L_{2}$ are $\xi$ transcendental. Find classes $v_{0} \in H^{*}\left(X ; L_{1}\right), v_{1}, \ldots, v_{k} \in H^{*}(X ; \mathbb{C}), u_{0} \in$ $H^{*}\left(Y ; L_{2}\right), u_{1}, \ldots, u_{r} \in H^{*}(Y ; \mathbb{C})$ such that $v_{0} \cup v_{1} \cup \cdots \cup v_{k} \neq 0$ and $u_{0} \cup u_{1} \cup \cdots \cup u_{r} \neq 0$. Now we have cohomology classes $v_{0} \times u_{0} \in H^{*}(X \times Y ; L)$ and $v_{i} \times 1,1 \times u_{j} \in H^{*}(X \times Y ; \mathbb{C})$ and the product

$$
\left(v_{0} \times u_{0}\right) \cup \prod_{i=1}^{k}\left(v_{i} \times 1\right) \cup \prod_{j=1}^{r}\left(1 \times u_{j}\right) \neq 0 \in H^{*}(X \times Y ; L)
$$


is nonzero. Here we use the Künneth formula which states

$$
H^{*}\left(X \times Y ; L_{1} \otimes L_{2}\right) \simeq H^{*}\left(X ; L_{1}\right) \otimes H^{*}\left(Y ; L_{2}\right) .
$$

This proves (67).

11.2. Some examples. In this section we apply Theorem 19 in a few simple examples.

11.2.1. First consider the case $\xi=0$. We know that for $\xi=0$ the number $\operatorname{cat}(X, \xi)$ coincides with the classical LS category cat $(X)$, see [18, Example 10.8. Let us examine what Theorem 19 gives in this case. The variety $\mathcal{V}_{\xi}$ has only one point - the trivial flat line bundle $\mathbb{C}$ over $X$. The support $\operatorname{Supp}(X, \xi)=\emptyset$ is always empty for $\xi=0$. We may therefore take $v_{0}=$ $1 \in H^{0}(X ; \mathbb{C})$ applying Theorem [19. Thus, we see that Theorem 19 claims in the special case $\xi=0$ that if there exist cohomology classes $v_{1}, \ldots, v_{k} \in$ $H^{>0}(X ; \mathbb{C})$ with $v_{1} \cup \cdots \cup v_{k} \neq 0$ then $\operatorname{cat}(X)>k$. This claim is the classical cup-length estimate for the LS category $\operatorname{cat}(X)$.

11.2.2. Note that if $\xi \neq 0$ and $X$ is connected then $H^{0}(X ; L)=0$ for any nontrivial $L \in \mathcal{V}_{\xi}$. Note also that the trivial flat line bundle $\mathbb{C} \in \mathcal{V}_{r}$ is never $\xi$-transcendental. Therefore the degree of the class $v_{0}$ (which appears in Theorem [19) in the case $\xi \neq 0$ must be positive. Hence for $\xi \neq 0$ the number $k$ in Theorem 19 satisfies $k \leq \operatorname{dim} X-1$. This explains why Theorem 19 cannot give $\operatorname{cat}(X, \xi) \geq \operatorname{dim} X+1$ for $\xi \neq 0$.

Lemma 10 yields

$$
\operatorname{cat}(X, \xi) \leq \operatorname{cat}(X)-1 \leq \operatorname{dim} X
$$

assuming that $X$ is connected and $\xi \neq 0$. This is consistent with the remark of the previous paragraph.

11.2.3. The following example shows that (69) can be satisfied as an equality i.e. that $\operatorname{cat}(X, \xi)=\operatorname{dim} X$ is possible. Consider the bouquet $X=Y \vee S^{1}$ where $Y$ is a finite polyhedron, and assume that the class $\xi \in H^{1}(X ; \mathbb{R})$ satisfies $\left.\xi\right|_{Y}=0$ and $\left.\xi\right|_{S^{1}} \neq 0$. We know that in this case

$$
\operatorname{cat}(X, \xi)=\operatorname{cat}(Y)-1,
$$

see Example 10.11 from [18.

We are going to apply Theorem [19, The variety $\mathcal{V}_{\xi}$ in this case coincides with the set $\mathbb{C}^{*}=\mathbb{C}-\{0\}$. The support $\operatorname{Supp}(X, \xi)$ contains in this case only the trivial line bundle. $L \in \mathcal{V}_{\xi}$ is $\xi$-transcendental if the monodromy along the circle $S^{1}$ is a $\xi$-transcendental complex number. For any $L \in \mathcal{V}_{\xi}$ the restriction $\left.L\right|_{Y}$ is trivial and the restriction homomorphism $H^{i}(X ; L) \rightarrow$ $H^{i}(Y ; \mathbb{C})$ is onto.

Suppose that the cohomological cup-length of $Y$ with $\mathbb{C}$-coefficients equals $\ell$, i.e. there exist cohomology classes of positive degree $u_{0}, u_{1}, \ldots, u_{\ell-1} \in$ $H^{>0}(Y ; \mathbb{C})$ such that the product $u_{0} \cup \cdots \cup u_{\ell-1} \neq 0$ is nonzero. By the above 
remark, for a nontrivial $L \in \mathcal{V}_{\xi}$ we obtain cohomology classes $v_{0} \in H^{*}(X ; L)$ and $v_{1}, \ldots, v_{\ell-1} \in H^{>0}(X ; \mathbb{C})$ such that $\left.v_{i}\right|_{Y}=u_{i}$. Hence $v_{0} \cup v_{1} \cup \cdots \cup$ $v_{\ell-1} \neq 0 \in H^{*}(X ; L)$. By Theorem 19 we obtain $\operatorname{cat}(X, \xi)>\ell-1$ which is equivalent (taking into account (170)) to $\operatorname{cat}(Y)>\ell$. The last inequality is the classical cup-length estimate for the usual category.

11.2.4. Let us now consider a very specific example: $X=T^{2} \vee S^{1}$. In this case $H^{1}(X ; \mathbb{R})=\mathbb{R}^{3}$ and we describe $\operatorname{cat}(X, \xi)$ as function of $\xi \in$ $H^{1}(X ; \mathbb{R})=\mathbb{R}^{3}$. We denote by $\ell \subset \mathbb{R}^{3}$ the set of all classes $\xi$ such that $\left.\xi\right|_{T^{2}}=0$. Clearly $\ell$ is a line through the origin in $\mathbb{R}^{3}$. We claim that:

$$
\operatorname{cat}(X, \xi)= \begin{cases}1, & \text { if } \xi \in \mathbb{R}^{3}-\ell, \\ 2, & \text { if } \xi \in \ell-\{0\} \\ 3, & \text { if } \xi=0\end{cases}
$$

Indeed, consider first the case $\xi \notin \ell$, i.e. $\left.\xi\right|_{T^{2}} \neq 0$. Let us show that $\operatorname{cat}(X, \xi) \leq 1$. Denote $p=T^{2} \cap S^{1}$ and let $q \in S^{1}$ be a point distinct from p. Set $F=X-\{q\}$ and $F_{1}=S^{1}-\{p\}$. Then $F \cup F_{1}=X$ is an open cover of $X$ with $F_{1} \rightarrow X$ null-homotopic and with $F$ being $N$-movable in $X$ for any $N$ (assuming that $\left.\xi\right|_{T^{2}} \neq 0$; this follows from homotopy invariance of $\operatorname{cat}(X, \xi)$ and from Example 1 .

Since $\operatorname{cat}(X, \xi)=0$ would imply $\chi(X)=0$ by Theorem 7 stated below we obtain that $\operatorname{cat}(X, \xi)>0$ for any $\xi($ as $\chi(X)=-1 \neq 0)$. This proves the first line of (71).

If $\xi \in \ell-\{0\}$ we apply (70) to obtain $\operatorname{cat}(X, \xi)=\operatorname{cat}\left(T^{2}\right)-1=2$.

For $\xi=0$ we easily find $\operatorname{cat}(X, \xi)=\operatorname{cat}(X)=3$.

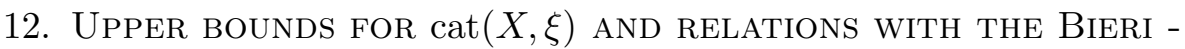 NEUMANN - StREBEL INVARIANTS}

Bieri, Neumann and Strebel introduced in 1 a geometric invariant of discrete groups $G$ which captures information about the finite generation of kernels of abelian quotients of $G$. In this section we describe a relation between this invariant and properties of $\operatorname{cat}(X, \xi)$.

Let us recall the definition. We always assume that $G$ is finitely presented as this is sufficient for our purposes. Let $S(G)$ denote $(\operatorname{Hom}(G, \mathbb{R})-\{0\}) / \mathbb{R}_{+}$ where $\mathbb{R}_{+}$acts on $\operatorname{Hom}(G, \mathbb{R})$ by multiplication. Clearly $S(G)$ is a sphere of dimension $n-1$ where $n$ is the rank of the abelianization of $G$. Denote by $[\chi]$ the equivalence class of a nonzero homomorphism $\chi: G \rightarrow \mathbb{R}$. The BieriNeumann-Strebel invariant associates to $G$ a subset $\Sigma(G) \subset S(G)$ defined as follows. Let $X$ be a finite cell complex with $\pi_{1}(X)=G$ and let $p: \tilde{X} \rightarrow X$ be the universal abelian cover of $X$. A homomorphism $\chi \in \operatorname{Hom}(G, \mathbb{R})$ can be viewed as a cohomology class lying in $H^{1}(X ; \mathbb{R})$. One has $\chi \in \Sigma(G)$ if and

\footnotetext{
${ }^{4}$ We rely on Theorem 5.1 of 1 ] which states that the definition of $\Sigma(G)$ given above coincides in the case of finitely presented $G$ with the main definition of [1].
} 
only if the inclusion $N \rightarrow \tilde{X}$ induces an epimorphism $\pi_{1}\left(N, x_{0}\right) \rightarrow \pi_{1}\left(\tilde{X}, x_{0}\right)$ where $N \subset \tilde{X}$ is a connected neighborhood of infinity with respect to $\chi$, see $\S 2$ of [24] and Lemma 5.2 from [1].

The following Theorem summarizes the results proven in [24]:

Theorem 21 (Farber - Schütz).

(a) Let $X$ be a finite connected polyhedron and let $\xi \in H^{1}(X ; \mathbb{R})$ be nonzero. Then

$$
\operatorname{cat}(X, \xi) \leq \operatorname{dim} X
$$

(b) Let $M$ be a closed connected smooth manifold and let $\xi \in H^{1}(M ; \mathbb{R})$ be nonzero. Then

$$
\operatorname{cat}(M, \xi) \leq \operatorname{dim}(M)-1
$$

(c) Let $M$ be a closed connected smooth manifold of dimension $\geq 5$ and let $\xi \in H^{1}(M ; \mathbb{R})$ be nonzero. If either

$$
\xi \in \Sigma\left(\pi_{1}(M)\right) \quad \text { or } \quad-\xi \in \Sigma\left(\pi_{1}(M)\right)
$$

then

$$
\operatorname{cat}(M, \xi) \leq \operatorname{dim} M-2 .
$$

(d) Let $M$ be a closed connected smooth manifold of dimension $\geq 5$ and let $\xi \in H^{1}(M ; \mathbb{R})$ be nonzero. If both inclusions take place

$$
\xi \in \Sigma\left(\pi_{1}(M)\right) \quad \text { and } \quad-\xi \in \Sigma\left(\pi_{1}(M)\right)
$$

then

$$
\operatorname{cat}(M, \xi) \leq \operatorname{dim} M-3
$$

13. Homological CATEgory Weights, estimates for $\operatorname{cat}^{1}(X, \xi)$ And CALCUlation of $\operatorname{cat}(X, \xi), \operatorname{cat}^{1}(X, \xi)$ FOR PRODUCTS OF SURFACES

In this section we describe briefly the results obtained in [25] which has as its main goal obtaining cohomological lower estimates for $\operatorname{cat}^{1}(X, \xi)$. In particular we will see that there are many examples when $\operatorname{cat}(X, \xi)<\operatorname{cat}^{1}(X, \xi)$ and the difference $\operatorname{cat}^{1}(X, \xi)-\operatorname{cat}(X, \xi)$ can be arbitrarily large.

The first step is the introduction of a new notion of category weight of homology classes which is somewhat dual to the cohomological notion introduced by E. Fadell and S. Husseini [8]. It was a pleasant surprise to discover that the homological category weight is homotopy invariant unlike the cohomological version of Fadell and Husseini.

The notion of homological category weight allows us to obtain improved cohomological lower bounds for $\operatorname{cat}^{1}(X ; \xi)$. 
13.1. Homological category weight. The classical cohomological lower bound for the Lusternik - Schnirelmann category cat $(X)$ states that $\operatorname{cat}(X)>$ $n$ if there exist $n$ cohomology classes of positive degree $u_{j} \in H^{*}\left(X ; R_{j}\right)$ where $j=1,2, \ldots, n$, such that their cup-product $u_{1} u_{2} \ldots u_{n} \neq 0 \in H^{*}(X ; R)$ is nontrivial. Here $R_{j}$ denotes a local coefficient system on $X$ and $R$ is the tensor product $R=R_{1} \otimes \cdots \otimes R_{n}$.

E. Fadell and S. Husseini [8] improved this estimate by introducing the notion of a category weight $\operatorname{cwgt}(u)$ of a cohomology class $u \in H^{q}(X ; R)$.

Definition 13 (Fadell - Husseini, [8]). Let $u \in H^{q}(X ; R)$ be a nonzero cohomology class where $R$ is a local coefficient system on $X$. One says that $\operatorname{cwgt}(u) \geq k$ (where $k \geq 0$ is an integer) if for any closed subset $A \subset X$ with cat $_{X} A \leq k$ one has $\left.u\right|_{A}=0 \in H^{q}(A ; R)$.

Recall that the inequality $\operatorname{cat}_{X} A \leq k$ means that $A$ can be covered by $k$ open subsets $U_{i} \subset X$ such that each inclusion $U_{i} \subset X$ is null-homotopic, $i=1, \ldots, k$.

According to Definition 13 one has $\operatorname{cwgt}(u) \geq 0$ in general and $\operatorname{cwgt}(u) \geq 1$ for any nonzero cohomology class of positive degree. As Fadell and Husseini [8] showed, $\operatorname{cwgt}(u)>1$ in some special situations which improves the lower estimate for $\operatorname{cat}(X)$. Indeed, one has

$$
\operatorname{cat}(X) \geq 1+\sum_{i=1}^{n} \operatorname{cwgt}\left(u_{i}\right)
$$

assuming that the cup-product $u_{1} u_{2} \ldots u_{n} \neq 0$ is nonzero.

Y. Rudyak [51] and J. Strom [59] studied a modification of $\operatorname{cwgt}(u)$, called the strict category weight $\operatorname{swgt}(u)$. The latter has the advantage of being homotopy invariant. However in some examples the strict category weight is considerably smaller than the original category weight of Fadell and Husseini.

In 25] we introduced and exploited a "dual" notion of category weight of homology classes. It has the geometric simplicity and clarity of category weight as defined by Fadell and Husseini and has a surprising advantage of being homotopy invariant.

Definition 14 (Farber - Schütz, [25]). Let $z \in H_{q}(X ; R)$ be a singular homology class with coefficients in a local system $R$ and let $k \geq 0$ be a nonnegative integer. We say that $\operatorname{cwgt}(z) \geq k$ if for any closed subset

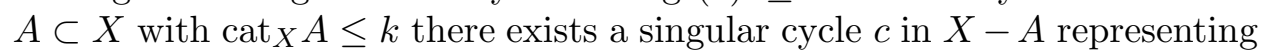
$z$. We say that $\operatorname{cwgt}(z)=k$ iff $\operatorname{cwgt}(z) \geq k$ and $\operatorname{cwgt}(z) \geq k+1$.

In other words, $\operatorname{cwgt}(z) \geq k$ is equivalent to the fact that $z$ can be realized

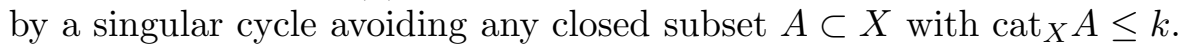

For example, $\operatorname{cwgt}(z) \geq 1$ iff $z$ can be realized by a singular cycle avoiding any closed subset $A \subset X$ such that the inclusion $A \rightarrow X$ is homotopic to a constant map. 
It will be convenient to define the category weight of the zero homology class as $+\infty$.

Formally $\operatorname{cwgt}(z) \geq k$ if $z$ lies in the intersection

$$
\bigcap_{A} \operatorname{Im}\left[H_{q}(X-A ; R) \rightarrow H_{q}(X ; R)\right]
$$

where $A \subset X$ runs over all closed subsets with $\operatorname{cat}_{X} A \leq k$.

The relation $\operatorname{cwgt}(z) \leq k$ means that there exists a closed subset $A \subset X$ with $\operatorname{cat}_{X} A \leq k+1$ such that any geometric realization of $z$ intersects $A$. In particular we obtain the following inequality

$$
\operatorname{cat}(X) \geq \operatorname{cwgt}(z)+1
$$

for any nonzero homology class $z \in H_{q}(X ; R), z \neq 0$. The last inequality can be also rewritten as

$$
0 \leq \operatorname{cwgt}(z) \leq \operatorname{cat}(X)-1 \leq \operatorname{dim} X
$$

for any nonzero homology class.

Note that if $X$ is path-connected and $z$ is zero-dimensional, i.e. $z \in H_{0}(X)$, then $\operatorname{cwgt}(z)=\operatorname{cat}(X)-1$.

Lemma 24. Assume that $X$ is a simplicial polyhedron. Then $\operatorname{cwgt}(z) \geq k$

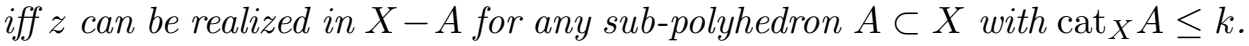

Proof. We only need to show the 'if'-direction. Let $A \subset X$ be closed with cat $_{X} A \leq k$. We need to show that $z$ can be realized by a cycle in $X-A$. We have $A \subset U_{1} \cup \cdots \cup U_{k}$ with each $U_{i}$ open and null-homotopic in $X$. Passing to a fine subdivision of $X$, we can find a sub-polyhedron $B \subset X$ with $A \subset B \subset U_{1} \cup \cdots \cup U_{k}$. Then cat $_{X} B \leq k$ and $z$ can be realized by a cycle lying in $X-B \subset X-A$.

Example 2. Assume that $X$ is a closed 2-dimensional manifold, i.e. a compact surface. Let us show that any nonzero homology class $z \in H_{1}(X)$ has $\operatorname{cwgt}(z) \geq 1$. Indeed, it is easy to see that any closed subset $A \subset X$ which is null-homotopic in $X$ lies in the interior of a disk $D^{2} \subset X$; but $H_{1}\left(X-\operatorname{Int} D^{2}\right) \rightarrow H_{1}(X)$ is an isomorphism.

Theorem 22 (Farber - Schütz, 25]). If $f: X \rightarrow Y$ is a homotopy equivalence then for any homology class $z \in H_{q}(X ; R)$ one has

$$
\operatorname{cwgt}(z)=\operatorname{cwgt}\left(f_{*}(z)\right) \text {. }
$$

Here $f_{*}(z) \in H_{q}\left(X ; R^{\prime}\right)$ where $R^{\prime}=g^{*} R$ is the local coefficient system over $Y$ induced by the homotopy inverse $g: Y \rightarrow X$ of $f$.

Another important result from [25] shows how one may use the notion of homological category weight in conjunction with the notion of Fadell and Husseini to estimate $\operatorname{cat}(X)$ : 
Corollary 25. Suppose that $X$ is a metric space and for some classes $z \in$ $H_{q}(X ; R)$ and $u \in H^{q}\left(X ; R^{\prime}\right)$ the evaluation

$$
\langle u, z\rangle \neq 0 \in R^{\prime} \otimes R
$$

is nonzero. Then

$$
\operatorname{cat}(X) \geq \operatorname{cwgt}(z)+\operatorname{cwgt}(u)+1 .
$$

Here $\operatorname{cwgt}(z)$ is the category weight of homology class $z$ as defined above and $\operatorname{cwgt}(u)$ is the category weight of $u$ as defined by Fadell and Husseini [8].

The main idea standing behind this Corollary is that "the degree of nontriviality" of a cohomology class u can be "measured" by "quality" of the homology class z satisfying $\langle u, z\rangle \neq 0$.

It is curious to observe that in the case of closed manifolds our notion of category weight of homology classes coincides with the cohomological notion of Fadell and Husseini [8] via the Poincarè duality. However for Poincaré complexes these notions are distinct as we show by an example given below.

Theorem 23 (Farber - Schütz, 25]). Suppose that $X$ is a closed n-dimensional manifold, $z \in H_{q}(X ; R)$ where $R$ is a local coefficient system. Let $u \in H^{n-q}(X ; R \otimes \tilde{\mathbb{Z}})$ be the Poincaré dual cohomology class, i.e. $z=u \cap[X]$, see below. Then

$$
\operatorname{cwgt}(z)=\operatorname{cwgt}(u)
$$

Here $\tilde{\mathbb{Z}}$ denotes the orientation local system on $X$, i.e. for a point $x \in X$ the stalk of $\tilde{\mathbb{Z}}$ at $x$ is $\tilde{\mathbb{Z}}_{x}=H_{n}(X, X-x ; \mathbb{Z})$, see $[58]$.

Example 3. Let $X=\mathbf{R P}^{n}$ be the real projective space. For the unique nonzero cohomology class $z \in H_{q}\left(X ; \mathbb{Z}_{2}\right)$ one has $\operatorname{cwgt}(z)=n-q$. Indeed, the dual homology class is $\alpha^{n-q} \in H^{n-q}\left(X ; \mathbb{Z}_{2}\right)$ where $\alpha \in H^{1}\left(X ; \mathbb{Z}_{2}\right)$ is the generator. Clearly, $\operatorname{cwgt}\left(\alpha^{n-q}\right)=n-q$.

Theorem 23 implies:

Corollary 26. If $X$ is a closed $n$-dimensional manifold then for any homology class $z \in H_{q}(X ; R)$ with $q<n$ one has

$$
\operatorname{cwgt}(z) \geq 1 \text {. }
$$

Indeed, if $q<n$ then the dual cohomology class $u$ has positive degree and hence $\operatorname{cwgt}(u) \geq 1$.

Consider now the case when $X$ is $n$-dimensional Poincaré complex. The following example shows that Theorem 23 is false for Poincaré complexes. It is a modification of an argument due to D. Puppe showing that the notion of category weight of cohomology classes is not homotopy invariant. 
Example 4. Consider the lens space $L=S^{2 n+1} /(\mathbb{Z} / p)$ where $p$ is an odd prime and $\mathbb{Z} / p$ acts freely on $S^{2 n+1}$. Denote by $r: S^{2 n+1} \rightarrow L$ the quotient map. Let $X$ be the mapping cylinder of $r$, i.e.

$$
X=L \sqcup S^{2 n+1} \times[0,1] / \sim
$$

where each point $(x, 0) \in S^{2 n+1} \times[0,1]$ is identified with $r(x) \in L$. Clearly $X$ is homotopy equivalent to $L$ and so it is a Poincaré complex. By a theorem of Krasnoselski [29], the category of $X$ equals $2 n+2$. Hence for $z=1 \in H_{0}\left(X ; \mathbb{Z}_{2}\right)$ one has

$$
\operatorname{cwgt}(z)=\operatorname{cat}(X)-1=2 n+1,
$$

see above. The dual cohomology class $u$ is the generator $u \in H^{2 n+1}\left(X ; \mathbb{Z}_{2}\right)$. Let us show that

$$
\operatorname{cwgt}(u)=1 \text {. }
$$

Indeed, consider the sphere $S=S^{2 n+1} \times 1 \subset X$. The restriction $\left.u\right|_{S} \in$ $H^{2 n+1}\left(S ; \mathbb{Z}_{2}\right)$ coincides with the induced class $r^{*}(v)$ where $v \in H^{2 n+1}\left(L ; \mathbb{Z}_{2}\right)$ is the generator. Hence the cohomology class $\left.u\right|_{S}$ is nonzero. However, the sphere $S$ has category 2 and moreover cat $_{X} S=2$ (as the inclusion $S \rightarrow X$ is not null-homotopic).

13.2. Cohomological estimate for $\operatorname{cat}^{1}(X, \xi)$. The following Theorem is the main result established in [25].

Theorem 24 (Farber - Schütz, 25]). Let $X$ be a finite cell complex and $\xi \in H^{1}(X ; \mathbb{R})$. Let $L \in \mathcal{V}_{\xi}$ be a complex flat line bundle over $X$ which is not a $\xi$-algebraic integer (see Definition 12). Suppose that for an integral homology class $z \in H_{q}(\tilde{X} ; \mathbb{Z})=H_{q}(X ; \Lambda)$ and some cohomology classes $u \in$ $H^{d}(X ; L)$ and $u_{i} \in H^{d_{i}}(X ; \mathbb{C})$, where $d_{i}>0$ for $i=1, \ldots, k$, the evaluation $\left\langle u \cup u_{1} \cup \cdots \cup u_{k}, p_{*}(z)\right\rangle \neq 0 \in \mathbb{C}$ is nonzero. Here $p_{*}(z) \in H_{d}\left(X ; L^{*}\right)$, $q=d+d_{1}+\cdots+d_{k}$. Then one has

$$
\operatorname{cat}^{1}(X, \xi) \geq \operatorname{cwgt}(z)+k+1 \text {. }
$$

Here $\operatorname{cwgt}(z)$ denotes the category weight of $z$ viewed as a homology class of $X$ with local coefficient system $\Lambda=\mathbb{Z}[H]$ where $H=H_{1}(X ; \mathbb{Z}) / \operatorname{Ker}(\xi)$ is a free abelian group which can be identified with the group of periods of $\xi$.

13.3. $\operatorname{cat}(X, \xi)$ and $\operatorname{cat}^{1}(X, \xi)$ for products of surfaces.

Theorem 25 (Farber - Schütz [24, 25]). Let $M^{2 k}$ denote the product

$$
\Sigma_{1} \times \Sigma_{2} \times \cdots \times \Sigma_{k}
$$

where each $\Sigma_{i}$ is a closed orientable surface of genus $g_{i}>1$. Given a cohomology class $\xi \in H^{1}\left(M^{2 k} ; \mathbb{R}\right)$, one has

$$
\operatorname{cat}\left(M^{2 k}, \xi\right)=1+2 r
$$

and

$$
\operatorname{cat}^{1}\left(M^{2 k}, \xi\right)=1+k+r
$$


where $r=r(\xi)$ denotes the number of indices $i \in\{1,2, \ldots, k\}$ such that the cohomology class $\left.\xi\right|_{\Sigma_{i}} \in H^{1}\left(\Sigma_{i}, \mathbb{R}\right)$ vanishes. In particular

$$
\operatorname{cat}\left(M^{2 k}, \xi\right)=1
$$

and

$$
\operatorname{cat}^{1}\left(M^{2 k}, \xi\right)=1+k
$$

assuming that $\left.\xi\right|_{\Sigma_{i}} \neq 0 \in H^{1}\left(\Sigma_{i} ; \mathbb{R}\right)$ for any $i=1, \ldots, k$.

We refer the reader to our papers [24, 25] for proofs and more detail.

Theorem 25 demonstrates that the difference between two notions of category with respect to a cohomology class

$$
\operatorname{cat}^{1}(X, \xi)-\operatorname{cat}(X, \xi)
$$

can be arbitrarily large.

Some further results developing the Lusternik-Schnirelman theory for closed 1-forms were obtained by J. Latschev [31] and D. Schütz [60]; see also [16] and [17].

\section{REFERENCES}

[1] R. Bieri, W. Neumann, R. Strebel, A geometric invariant of discrete groups, Invent. Math. 90 (1987), 451-477.

[2] R. Bieri, B. Renz, Valuations on free resolutions and higher geometric invariants of groups, Comment. Math. Helv. 63 (1988), 464-497.

[3] P. M. Cohn, Free rings and their relations, Academic Press, London, 1985.

[4] C. Conley, Isolated invariant sets and the Morse index, CBMS Regional Conference Series in Mathematics, no. 38, AMS, 1976.

[5] C. Conley, The gradient structure of a flow: I, Ergod. Th. \& Dynam. Sys., 8(1988), $11-26$.

[6] A. Dold, Lectures on Algebraic topology, Springer-Verlag, 1972.

[7] B. Dubrovin, S. Novikov and A. Fomenko, Modern Geometry; Methods of the homology theory, 1984.

[8] E. Faddell, S. Husseini, Category weight and Steenrod operations, Boletín de la Sociedad Mathemática Mexicana, 37(1992), 151-161

[9] H. Fan, J. Jost, Novikov-Morse theory for dynamical systems. Calc. Var. Partial Differential Equations 17 (2003), no. 1, 29-73.

[10] H. Fan, J. Jost, Conley index theory and Novikov-Morse theory. Pure Appl. Math. Q. 1 (2005), no. 4, part 3, 939-971.

[11] M. Farber, Exactness of Novikov inequalities, Functional Analysis and its Applications 19(1985), 40-48.

[12] M. Farber and A. Ranicki, The Morse-Novikov theory of circle-valued functions and noncommutative localization, Proceedings of the Steklov Institute of Mathematics, 225(1999), pp. 363 - 371.

[13] M. Farber, Morse-Novikov critical point theory, Cohn localization and Dirichlet units, Communications in Contemporary Mathematics, 1(1999), 467 - 495.

[14] M. Farber, Counting zeros of closed 1-forms, "Topology, Ergodic Theory, Real Algebraic Geometry. Rokhlin's Memorial", V. Turaev, A. Vershik editors, Amer. Math. Translations, Ser. 2, 202(2001), 95 - 107.

[15] M. Farber, Zeros of closed 1-forms, homoclinic orbits and Lusternik-Schnirelman theory, Topol. Methods Nonlinear Anal. 19 (2002), 123-152. 
[16] M. Farber, Lusternik-Schnirelman theory and dynamics, "Lusternik-Schnirelmann Category and Related Topics", O. Cornea, G. Lupton, J. Oprea and D. Tanré editors, Contemporary Mathematics, 316(2002), 95 - 111.

[17] M. Farber, T. Kappeler, Lusternik-Schnirelmann theory and dynamics, II, Proc. Steklov Inst. Math. 2004, no. 4 (247), 232-245.

[18] M. Farber, Topology of closed one-forms, Mathematical Surveys and Monographs, AMS, 2004.

[19] M. Farber, T. Kappeler, J. Latschev, E. Zehnder, Lyapunov 1-forms for flows, "Ergodic theory and dynamical systems", 24(2004), 1451-1475.

[20] M. Farber, T. Kappeler, J. Latschev, E. Zehnder, Smooth Lyapunov 1-forms, "L'Enseignement Mathematique", 50 (2004), no. 1-2, 3-17.

[21] M. Farber and D. Schütz, Novikov Betti numbers and the fundamental group, Russian Math. Surveys 61 (2006), no. 6, pp. 1173-1175.

[22] M. Farber, D. Schütz, Closed 1-forms with at most one zero, Topology, 45(2006), 465-473.

[23] M. Farber, D. Schütz, Moving homology classes to infinity, Forum Math. 19 (2007), 281-296.

[24] M. Farber, D. Schütz, Cohomological estimates for cat $(X, \xi)$, Geom. Top. 11 (2007), 1255-1289.

[25] M. Farber, D. Schütz, Homological category weights and estimates for $\operatorname{cat}^{1}(X, \xi)$, J. Eur. Math. Soc. 10 (2008), 243-266.

[26] M. Farber, R. Geoghegan and D. Schütz, Closed 1-forms in topology and geometric group theory, preprint.

[27] D. Fried, The geometry of cross sections to flows, Topology, 21(1982), 353-371.

[28] H. Hofer and D. Salamon, Floer homology and Novikov rings, Floer memorial volume, Birkhäuser (1995), pp. 483-524.

[29] M.A. Krasnoselski, On special coverings of finite dimensional sphere, Doklady AN SSSR, 103(1955), 961-964.

[30] S. Lang, Real and Functional Analysis, third edition, Graduate Texts in Mathematics, 142, 1993, Springer-Verlag

[31] J. Latschev, Flows with Lyapunov 1-forms and a generalization of Farber's Theorem on homoclinic cycles, "International Mathematical Research Notices", 2004:5, pp. 239-247.

[32] J. Latschev, Closed forms transverse to singular foliations, Manuscripta Math. 121 (2006), no. 3, 293-315.

[33] J. Latschev, Coherent measures and the existence of smooth Lyapunov 1-forms for flows, Topology 45 (2006), no. 4, 707-723.

[34] F. Latour, Existence de 1-formes fermées non singulières dans une classe de cohomologie de de Rham, Publ. IHES 80 (1994), 135-194.

[35] G. Levitt, 1-formes fermées singulières et groupe fondamental, Invent. Math., 88(1987), 635 - 667.

[36] S. P. Novikov, Multi-valued functions and functionals. An analogue of Morse theory, Soviet Math. Doklady, 24(1981), pp. 222-226.

[37] S. P. Novikov, I. Smel'tser, Periodic solutions of the Kirhhoff equations for the free motions of a rigid body in a liquid, and extended Lusternik-Schnirelman-Morse theory, I Functional Analysis and Its Applications, 15(1981), pp. 197 - 207.

[38] S. P. Novikov, Variational methods and periodic solutions of equations of Kirchhoff type, II, Functional Analysis and Its Applications, 15(1981), pp. 263 - 274.

[39] S.P. Novikov, The Hamiltonian formalism and a multi-valued analogue of Morse theory, Russian Math. Surveys 37 (1982), 1-56.

[40] S. P. Novikov, I. Taimanov, Periodic extremals in multivalued or not everywhere positive functionals, Soviet Math. Doklady, 29(1984), pp. 18 - 20. 
[41] S. P. Novikov, Bloch homology, critical points of functions and closed 1-forms, Soviet Math. Dokl., 33(1986), pp. 551-555.

[42] S. P. Novikov, Quasiperiodic structures in topology, Topological Methods in Modern Mathematics, Publish or Perish, 1993, pp. 223 - 235.

[43] Y.-G. Oh, Spectral invariants, analysis of the Floer moduli space and geometry of Hamiltonian diffeomorphisms, Duke Math. J., 130(2005), 199 - 295.

[44] A. Pazhitnov, On the sharpness of Novikov type inequalities for manifolds with free abelian fundamental group, Math. USSR Sbornik, 68(1991), pp. 351-389.

[45] A. Pazhitnov, Morse theory of closed 1-forms, Lecture Notes in Math, 1474(1991), pp. $98-110$

[46] A. V. Pazhitnov, On the Novikov complex of rational Morse forms, Ann. Fac. Sci. Toulouse Math., 4(1995), pp. 297 - 338.

[47] A. V. Pajitnov, Circle-valued Morse theory. de Gruyter Studies in Mathematics, 32. Walter de Gruyter \& Co., Berlin, 2006.

[48] A. Ranicki, Finite domination and Novikov rings, Topology, 34(1995), pp 619-632.

[49] A. Ranicki, The algebraic construction of the Novikov complex of a circle-valued Morse function, Mathematische Annalen 322(2002), 745-785.

[50] W. Rudin, Functional Analysis, McGraw-Hill Book Company, 1973.

[51] Y. Rudyak, On category weight and its applications, Topology 38(1999), pp. 37 -55.

[52] S. Schwartzman, Asymptotic cycles, Ann. of Math., 66(1957), pp. 270-284

[53] S. Schwartzman, Global cross-sections of compact dynamical systems, Proc. Nat. Acad. Sci. USA, 48(1962), pp. 786 - 791

[54] M. Shub, Global Stability of Dynamical Systems, Springer-Verlag, 1986.

[55] J.-Cl. Sikorav, Thèse, Université Paris-Sud, 1987.

[56] J.-Cl. Sikorav, Un problème de disjonction par isotopie symplectique dans un fibré cotangent, Ann. Sci. École Norm. Sup. (4)19(1986), 543-552.

[57] S. Smale, Stable manifolds for differential equations and diffeomorphisms, Ann. Scuola Norm. Sup. Pisa, 17(1963), 97 - 116.

[58] E.H. Spanier, Algebraic topology. McGraw-Hill Book Co., New York-Toronto, Ont.London 1966.

[59] J. Strom, Essential category weight and phantom maps, Cohomological methods in homotopy theory (Bellaterra, 1998), 409 - 415.

[60] D. Schütz, On the Lusternik-Schnirelman theory of a real cohomology class, Manuscripta Math. 113 (2004), 85-106.

[61] F. Takens, The minimal number of critical points of a function on a compact manifold and the Lusternik - Schnirelmann category, Invent. Math., 6(1968), pp. 197 - 244.

[62] M. Usher, Spectral numbers in Floer theories, preprint, available as arXiv:0709.1127

Department of Mathematics, University of Durham, Durham DH1 3LE, UK

E-mail address: Michael.Farber@durham.ac.uk

Department of Mathematics, University of Durham, Durham DH1 3LE, UK

E-mail address: dirk.schuetz@durham.ac.uk 